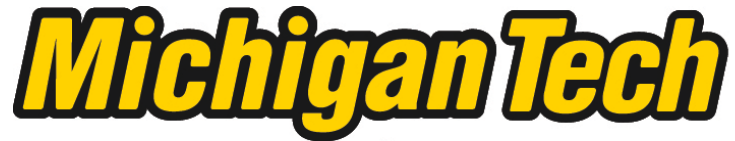 \\ Michigan Technological University Create the Future Digital Commons @ Michigan Tech
}

Dissertations, Master's Theses and Master's Reports - Open

Dissertations, Master's Theses and Master's

Reports

2012

Online distribution management framework by incorporating wind generation

Bhairavi Pandya

Michigan Technological University

Follow this and additional works at: https://digitalcommons.mtu.edu/etds

Part of the Electrical and Computer Engineering Commons

Copyright 2012 Bhairavi Pandya

\section{Recommended Citation}

Pandya, Bhairavi, "Online distribution management framework by incorporating wind generation", Master's Thesis, Michigan Technological University, 2012.

https://doi.org/10.37099/mtu.dc.etds/54

Follow this and additional works at: https://digitalcommons.mtu.edu/etds

Part of the Electrical and Computer Engineering Commons 


\title{
ONLINE DISTRIBUTION MANAGEMENT FRAMEWORK BY INCORPORATING WIND GENERATION
}

\author{
By \\ Bhairavi Pandya
}

\begin{abstract}
A THESIS
Submitted in partial fulfillment of the requirements for the degree of MASTER OF SCIENCE

(Electrical Engineering)
\end{abstract}

\section{MICHIGAN TECHNOLOGICAL UNIVERSITY}

2012

(C) 2012 Bhairavi Pandya 
This thesis, "Online Distribution Management Framework by Incorporating Wind Generation," is hereby approved in partial fulfillment of the requirements for the Degree of MASTER OF SCIENCE IN ELECTRICAL ENGINEERING.

Department of Electrical and Computer Engineering

Signatures:

Thesis Advisor

Dr. Chee-Wooi Ten

Committee Member

Dr. Leonard Bohmann

Committee Member

Dr. Laura Brown

Department Chair

Dr. Daniel Fuhrmann

Date 
This is dedicated to my beloved parents, Jatin and Niranjana Pandya, and my Mamasa, Mr. Navneetlal Attarwala, my pillars of strength. 


\section{Contents}

List of Figures $\ldots \ldots \ldots \ldots \ldots \ldots \ldots \ldots \ldots \ldots \ldots$ vii

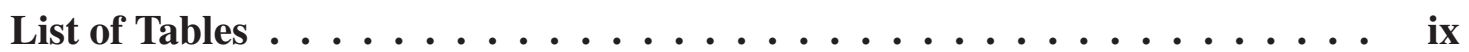

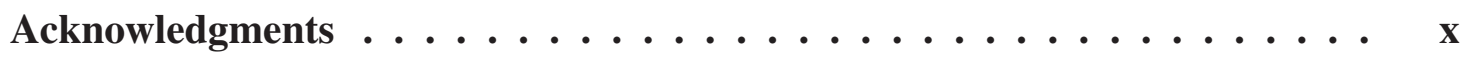

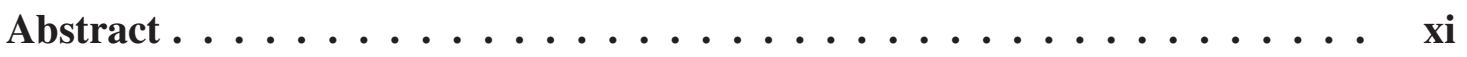

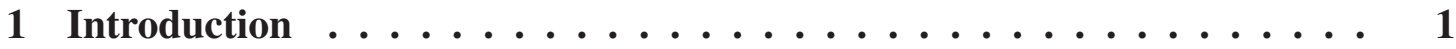

1.1 Future Distribution Systems . . . . . . . . . . . . . . . . . 2

1.2 Intermittent Distributed Generation in Distribution Systems . . . . . . . 4

1.3 Research Challenges and Motivations _... . . . . . . . . 5

1.4 Research Contributions . . . . . . . . . . . . . . . . . . . 7

1.5 The Remainder of the Thesis . . . . . . . . . . . . . . . 7

2 Background and Literature Review $\ldots \ldots \ldots \ldots \ldots$

2.1 Wind Speed Prediction $\ldots \ldots \ldots \ldots$

2.1.1 Scales of Prediction . . . . . . . . . . . . . . . . . . . . . . 9

2.1.2 Numerical Weather Predictions . . . . . . . . . . . . . . . . . 11

2.1.3 Statistical Models . . . . . . . . . . . . . . . . . . . . . . . 11

2.1 .4 Summary of Methods . . . . . . . . . . . . . . . . . . . 12

2.2 Wind Power . . . . . . . . . . . . . . . . . . . . 13

2.2.1 Coefficient of Performance and Betz Limit . . . . . . . . . . . . 14

2.3 Integration of Wind Generation . . . . . . . . . . . . . . 16

2.3.1 Wind Farm SCADA . . . . . . . . . . . . . . . . . . . 16

2.4 Distribution System Topologies . . . . . . . . . . . . . . . . . . 18

2.4.1 Primary Distribution System . . . . . . . . . . . . . . . . . . 19

2.4.2 Secondary Distribution System . . . . . . . . . . . . . . 21

2.5 Distribution System Reconfiguration . . . . . . . . . . . . . . 22

2.5.1 Remote Controllable Switches . . . . . . . . . . . . . . 22

2.5.2 Motivation for Reconfiguration . . . . . . . . . . . . . . 22 
2.5.3 Fault Location, Isolation, and Restoration . . . . . . . . . . . 25

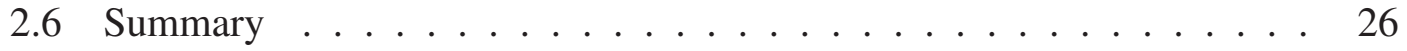

3 Prediction of Wind Speed . . . . . . . . . . . . . . . 27

3.1 Historical Data Analysis . . . . . . . . . . . . . . 28

3.1.1 Normalized Frequency Plots on Different Time-scales . . . . . . 30

3.2 Probabilities Using Parametric Statistic . . . . . . . . . . . . . . 33

3.2.1 Calculation of $k, c$ Parameters . . . . . . . . . . . . . 36

3.2.2 Simulation Results . . . . . . . . . . . . . . . . 39

3.3 Probabilities Using Nonparametric Statistics . . . . . . . . . . . . . . . 41

3.3.1 Simulation Results . . . . . . . . . . . . . . . . . 43

3.4 Comparison of Probabilities Calculated . . . . . . . . . . . . . . 44

3.5 Summary ......................... 45

4 Estimating Wind Generation Capacity . . . . . . . . . . . . . . 46

4.1 Turbine Power Output . . . . . . . . . . . . . . . . . . . 46

4.2 Average Power Output Prediction . . . . . . . . . . . . . . . . . . . . . 49

4.3 Simulation Results . . . . . . . . . . . . . . . . . . . . . . 50

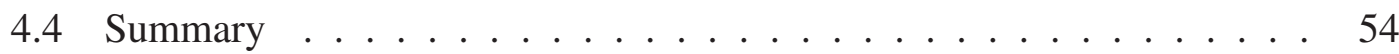

5 Enumeration of Switching Combinations . . . . . . . . . . . 55

5.1 Test System Description . . . . . . . . . . . . . . . . . . 57

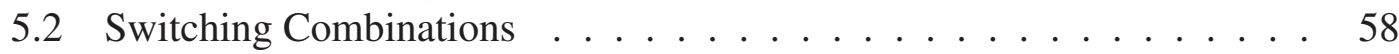

5.2.1 Possible Switching Combinations . . . . . . . . . . . 58

5.2.2 Feasible Switching Combinations . . . . . . . . . . . 59

5.2.3 Applying the Constraints . . . . . . . . . . . . . . . 60

5.3 System Setup . . . . . . . . . . . . . . . . . . . . . 67

5.3 .1 Required Data . . . . . . . . . . . . . . 68

5.4 Simulation Results . . . . . . . . . . . . . . . . . . . . 69

5.4.1 Normal Operating Conditions . . . . . . . . . . . . . . 70

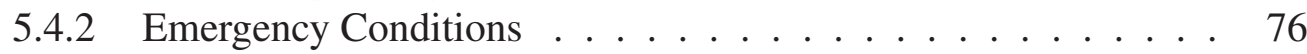

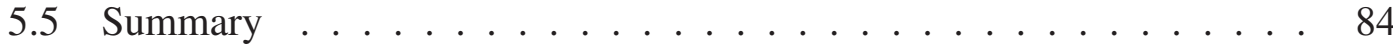

6 Results and Future Work ..................... 86

6.1 Major Research Contributions . . . . . . . . . . . . . . 86

6.2 Future Work . . . . . . . . . . . . . . . . . 87

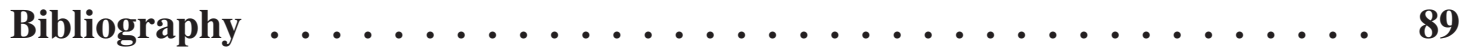

Appendix A Simulation Setup and Detailed Results . . . . . . . . . . 95

A.1 Wind Data Analysis . . . . . . . . . . . . . . 95 
A.2 Frequency Plots for Various Time-frames . . . . . . . . . . . . 95

A.3 Wind Probability Calculation . . . . . . . . . . . . . 96

A.4 Wind Power Calculation . . . . . . . . . . . . . . . 98

A.5 Test System Setup . . . . . . . . . . . . . . . . . . . . . . . . . . . . . . . . . . . . . . . . . .

A.6 Solver . . . . . . . . . . . . . . . . 103

A.7 Duration of Simulation . . . . . . . . . . . . . . 103

A.8 Monitoring and Protection Blocks . . . . . . . . . . . . 105

A.8.1 Distribution Substation Monitoring . . . . . . . . . . . . 106

A.8.2 Wind Farm Monitoring . . . . . . . . . . . . . . 106

A.8.3 Wind Farm Protection . . . . . . . . . . . . . . . 107

A.9 Simulation Results for Test System in MATLAB . . . . . . . . . . . . 108 


\section{List of Figures}

1.1 Proposed Approach . . . . . . . . . . . . . . . . . . 2

1.2 Wind Speed Variations Over A Day _. . . . . . . . . . . . . 5

1.3 Wind Speeds on a Monthly Average $\ldots \ldots \ldots \ldots$

2.1 Wind Power Forecasting Model . . . . . . . . . . . . . . . . 10

2.2 Wind Power Prediction . . . . . . . . . . . . . . . . . . 13

2.3 Mass Rate Flow . . . . . . . . . . . . . . . . . . . . . . 15

2.4 Wind SCADA . . . . . . . . . . . . . . . . . . 17

2.5 Primary Distribution System ～. . . . . . . . . . . . . . 20

2.6 Secondary Distribution System . . . . . . . . . . . . . . 21

3.1 Wind Speed Prediction Methodology . . . . . . . . . . . . . . 28

3.2 Total Data Points . . . . . . . . . . . . . . . . . . . . . . . . . 29

3.3 Data Resolution . . . . . . . . . . . . . . . . . . . . . . 30

3.4 Normalized Frequency Plot of Wind Speed Data . . . . . . . . . . . 31

3.5 Comparison of Number of Data Points . . . . . . . . . . . . . 32

3.6 Comparison of Extreme Bin Sizes . . . . . . . . . . . . . . 32

3.7 Weibull PDF for different Values of $k$ and $c \ldots \ldots \ldots \ldots$

3.8 PDF Curves Obtained from $k$ and $c$ Parameters . . . . . . . . . . 40

3.9 Wind Speeds with Highest Weibull Probability . . . . . . . . . . . . 41

3.10 Wind Speeds with Highest Nonparametric Probability . . . . . . . . . . 43

3.11 Comparison of Calculated Probabilities for All Wind Speeds . . . . . . 44

4.1 Estimating Wind Power Generation . . . . . . . . . . . . . . . 47

4.2 Output of a Wind turbine . . . . . . . . . . . . . . . 48

4.3 Simulated Output of the Wind turbine . . . . . . . . . . . . 51

4.4 Output of a Wind turbine from In-built MATLAB DFIG Block . . . . . 52

4.5 Predicted Average Hourly Output of the Wind Turbine - Weibull Proba-

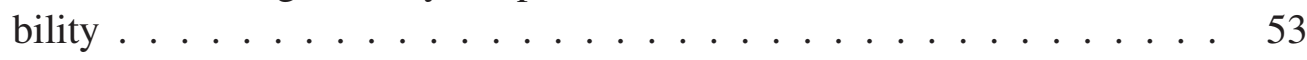

4.6 Predicted Average Hourly Output of the Wind Turbine - Nonparametric Probability . . . . . . . . . . . . . . . . . . 54 
5.1 Enumeration of Switching Combinations . . . . . . . . . . 56

5.2 Base Case with Wind DG . . . . . . . . . . . . . . 57

5.3 Feasible Switching Combinations Under Normal Operating Conditions . 62

5.4 Feasible vs. Non-feasible Switching Combination - Normal Conditions . 63

5.5 Feasible vs. Non-feasible Switching Combination - Emergency Conditions 64

5.6 Feasible Switching Combinations In case of an Outage at Feeder DS-1 . 65

5.7 Feasible Switching Combinations In case of an Outage at Feeder DS-2 . 66

5.8 Feasible Switching Combinations In case of an Outage at Feeder DS-3 . 67

5.9 Wind Farm-1 Output at $V=6 \mathrm{~m} / \mathrm{s} \ldots \ldots \ldots \ldots \ldots \ldots \ldots$

5.10 Wind Farm-1 Output at $V=14 \mathrm{~m} / \mathrm{s} \ldots \ldots \ldots \ldots \ldots \ldots$

5.11 Wind Farm-1 Output at $V=22 \mathrm{~m} / \mathrm{s} \ldots \ldots \ldots \ldots \ldots$

5.12 Wind Speed Probabilities and Relevant Output . . . . . . . . . . 74

5.13 DS-1 Outage: Full Output from Wind Farms-1 and $2 \ldots \ldots$. . . 77

5.14 DS-1 Outage: Low Output from Wind Farms-1 and $2 \ldots \ldots \ldots$

5.15 DS-2 Outage: Full Output from Wind Farms-1 and $2 \ldots \ldots . .79$

5.16 DS-2 Outage: Low Output from Wind Farms-1 and $2 \ldots \ldots \ldots$

5.17 DS-3 Outage: Full Output from Wind Farms-1 and $2 \ldots \ldots$

5.18 DS-3 Outage: Low Output from Wind Farms-1 and $2 \ldots \ldots \ldots$

A.1 Individual Normalized Frequency Plots for Each Season . . . . . . . . 96

A.2 Individual Normalized Frequency Plots for Each Year . . . . . . . . 97

A.3 Base Case MATLAB Configuration . . . . . . . . . . . . 100

A.4 MATLAB System Layout . . . . . . . . . . . . . . . . . . . 102

A.5 Monitoring and Control Blocks . . . . . . . . . . . . . . . 105

A.6 Wind Turbine Protection . . . . . . . . . . . . . . . . 107

A.7 Wind Farm Behavior in Absence of Grid Support . . . . . . . . . 109 


\section{List of Tables}

2.1 Wind Speed Prediction Techniques . . . . . . . . . . . . . 12

5.1 Cumulative Weibull Probabilities . . . . . . . . . . . . . . 75

5.2 Cumulative Nonparametric Probabilities . . . . . . . . . . . . 75

5.3 Cumulative Weibull Probabilities for Loss of Generation in Each Feeder 83

5.4 Cumulative Nonparametric Probabilities for Loss of Generation in Each Feeder . . . . . . . . . . . . . . . . . . 84

A.1 Calculated $k$ and $c$ Parameters $\ldots \ldots \ldots \ldots \ldots . \ldots \ldots$

A.2 Wind Speeds and Respective Probabilities for the Same Time of Day in a Season . . . . . . . . . . . . . . . . . . . . 99

A.3 Parameters Used to Calculate Individual Turbine Output . . . . . . . 100

A.4 Adjusted Wind Speeds and Respective Power Outputs for a Hub-height of $60 \mathrm{~m} \ldots \ldots \ldots \ldots \ldots 10 \ldots \ldots$

A.5 Time Duration for Simulation . . . . . . . . . . . . . . . . 104

A.6 Wind Farm Data . . . . . . . . . . . . . . . . . . . . . . . . . 104

A.7 Load Data for Test System ～. . . . . . . . . . . . . . . 104

A.8 Generation Data for Test System … . . . . . . . . . 105

A.9 Line Data for Test System . . . . . . . . . . . . . . . . . 105 


\section{Acknowledgments}

I would like to express my deepest and sincere gratitude to my supervisor, Dr. CheeWooi Ten for his encouragement, inspiration, deep insight and patience, without which this thesis would not have been possible.

I would also like to extend my gratitude to the committee members of this thesis, Dr. Leonard Bohmann and Dr. Laura Brown. I highly appreciate their precious time and efforts. Their valuable suggestions and comments have helped me improve the quality of this thesis. My gratitude goes to Dr. Dennis Wiitanen and Mr. John Lukowski for their support throughout my degree.

I would also like to take this opportunity to sincerely thank my research mates and friends, especially, Suryabh Sharma, Andrew Drees, and Elizaveta Egorova among several others. Discussions with them have proved to be vital, providing me with a lot of food for thought. Besides the ideas, I can never thank them enough for their incredible support and encouragement.

Last but not least, my deepest gratitude and kindest love goes to my family for their support and unwavering faith in me. There is no sufficient way in that I can express my gratitude towards everything they have done for me. 


\begin{abstract}
In distribution system operations, dispatchers at control center closely monitor system operating limits to ensure system reliability and adequacy. This reliability is partly due to the provision of remote controllable tie and sectionalizing switches. While the stochastic nature of wind generation can impact the level of wind energy penetration in the network, an estimate of the output from wind on hourly basis can be extremely useful. Under any operating conditions, the switching actions require human intervention and can be an extremely stressful task. Currently, handling a set of switching combinations with the uncertainty of distributed wind generation as part of the decision variables has been nonexistent. This thesis proposes a three-fold online management framework: (1) prediction of wind speed, (2) estimation of wind generation capacity, and (3) enumeration offeasible switching combinations. The proposed methodology is evaluated on 29 -node test system with 8 remote controllable switches and two wind farms of 18MW and 9MW nameplate capacities respectively for generating the sequence of system reconfiguration states during normal and emergency conditions.
\end{abstract}




\section{Chapter 1}

\section{Introduction}

Smart Grid concept has been central to the efforts of advancement of the electric grid towards a cyber-physical systems. In America, enormous infrastructure investment for renewable energy such as wind energy, biomass, fuel cells, or solar energy has been observed at every level from bulk transmission system to distribution system. Inclusion of intermittent generation in distribution systems can improve system reliability and adequacy; however, the complexity of operating states with stochastic nature of wind generation for system operations has yet to be fully explored. Inclusion of intermittent generation in distribution systems, especially wind energy, has introduced a new set of complexities in terms of system operation.

This thesis focuses particularly on operating aspects of a modern distribution system with wind generation. In subsequent sections of this chapter, an overview of future distribution systems, wind generation in distribution systems followed by research motivation and challenges involved is presented. The chapter is concluded with a list of research contributions of this work and organization of the rest of the thesis. 


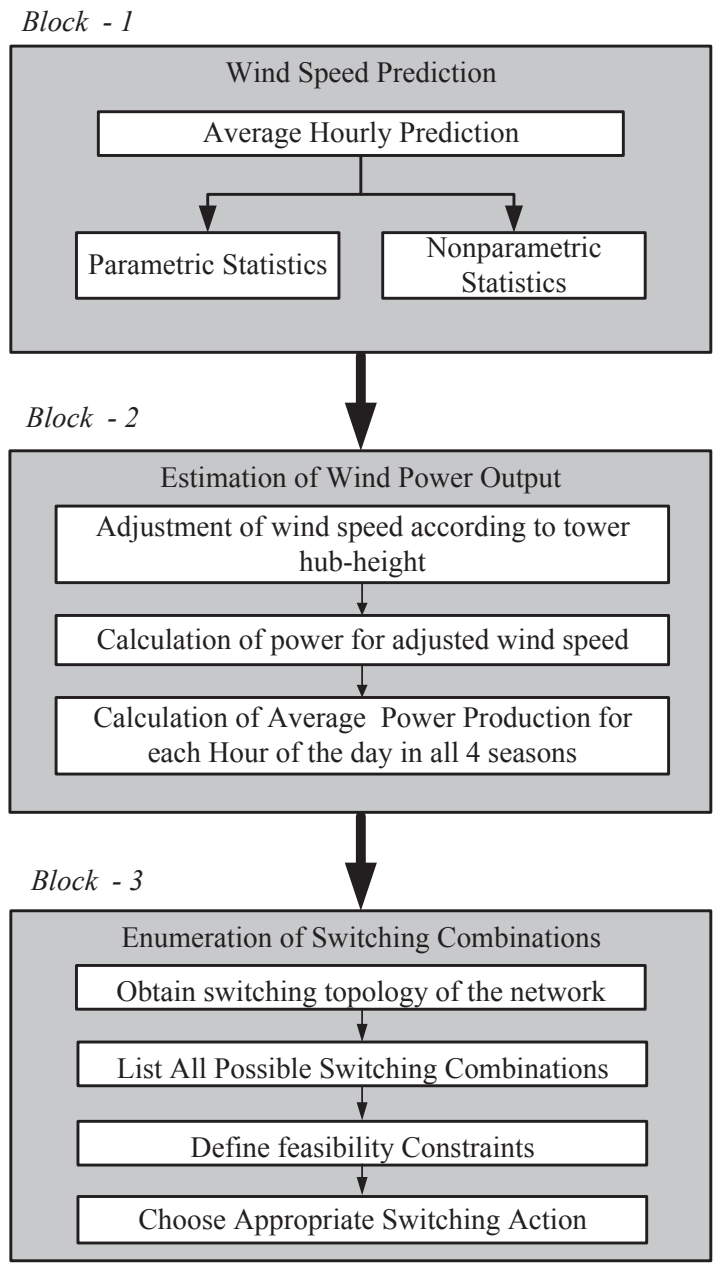

Figure 1.1 - Proposed Approach

\subsection{Future Distribution Systems}

Until recently, distribution systems have had little automation in terms of system operation. However, with the Smart Grid initiative this is changing. Distribution systems of the future [1] will be better equipped to cope with complexities introduced by wind generation with an interactive communication infrastructure, advanced metering and experience with high penetration levels of wind. The following are the goals of future 
distribution systems:

- an enhanced IP-based communication infrastructure,

- two-way communication among different parts of the system,

- dynamic pricing with customer participation,

- resilience against cyber-threats,

- a grid capable of automatically healing itself in events of faults and other disturbances,

- increased reliability and capacity in order to meet the ever-increasing power demand, and

- inter-operability.

Figure 1.1 describes the general approach taken towards the modeling of intermittent sources in the proposed distribution system. Each block defines each of the three goals and briefly describes the methodology employed to obtain them.

Block 1 describes wind speed prediction methodology. Probabilities of wind speeds observed are calculated using both parametric and nonparametric approaches. Both the approaches are also compared and contrasted.

Block 2 describes the output power calculation and incorporates the probabilities above calculated. Wind power output for the turbine model used is calculated and corresponding probabilities are included in form of weights to power calculation in order to estimate the capacity supplied by wind.

Finally, Block 3 describes a method to enumerate different switching combinations in small test system. The test system uses the calculated power output as input. All possible combinations of the 8 remote controllable switches present in the system are tested and feasible combinations are identified under normal operating conditions. Using the probabilities of various wind speeds, the probabilities of various relevant power outputs, and, thus, effective switching combinations are evaluated. 


\subsection{Intermittent Distributed Generation in Distribution Systems}

Radical changes in modern distribution systems are anticipated [2,3] as different novel alternatives to conventional central generation entities start gaining prominence. Most of these sources, if not all [4], exhibit different behavior from their bulk electricity producing counterparts. Renewable energy sources included in such distributed generation (DG) facilities are inherently highly intermittent even on a shorter time period. It can be vital to be able to predict short-term contingencies of a system with significant amount of DG in it.

According to [5], DG has no official definition. There exist several different definitions and DG can be loosely characterized by a capacity of few MWs and proximity of consumption site.

Inadvertently, these DG facilities at the distribution system level are introducing new challenges in system operation. Some of the apparent advantages of having DG facilities are:

- improved voltage profiles due to their additional voltage support [6],

- lower losses because of their proximity to the points of consumption $[7,8]$,

- insensitivity to fuel prices, given the driving agent is not fossil fuel for most of DG facilities [8], and

- little or no environmental concerns [9].

These apparent advantages of onsite renewable generating facilities like solar panels and wind turbines of small scale are only obtained after a careful planning of their placement and the amount of penetration. However, the topology of any distribution system is susceptible to autonomy of consumers. As a result some of the advantages mentioned above may not be achieved/exploited to their full potential. This is accompanied by the fact that DG facilities may, as a matter of fact, introduce complex problems on an operational front at the distribution level. Another important factor is the intermittency of renewable energy sources, in particular, DG facilities utilizing wind and solar energy. Among the named energy sources, wind has an especially highly intermittent nature. With increasing penetration of wind in the distribution systems, it is critical to be able to anticipate the behavior of DG facilities utilizing wind under all operating conditions. 


\subsection{Research Challenges and Motivations}

Motivation of this work is three-fold.

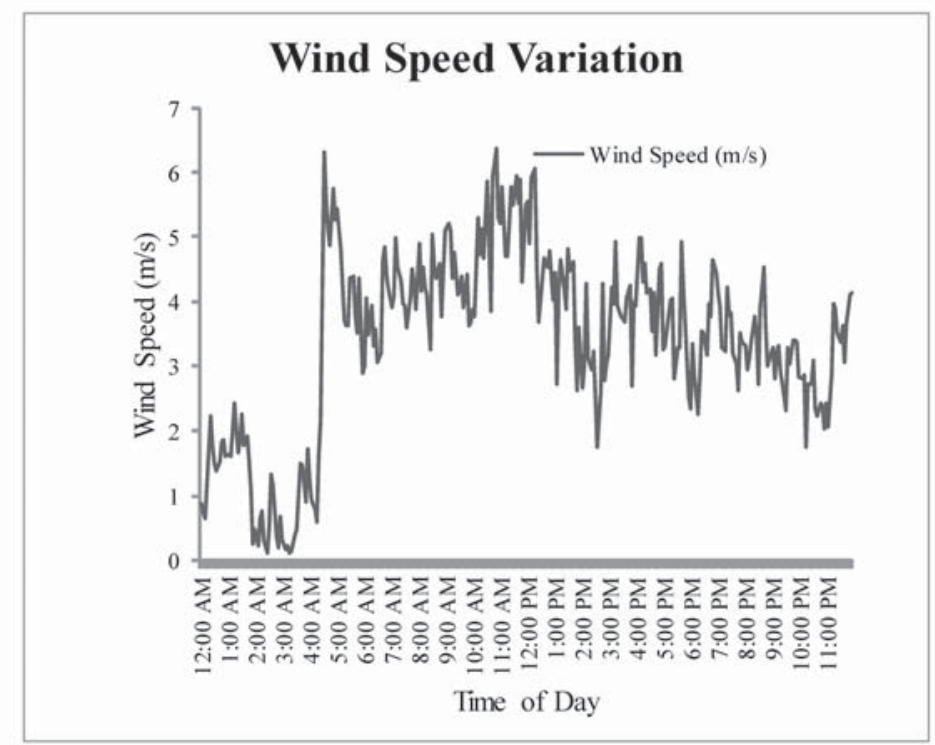

Figure 1.2 - Wind Speed Variations Over A Day

1. Wind speeds are highly intermittent and geography dependent. Techniques to accurately predict wind speeds on short-term basis exist in the field of meteorology. However, in meteorology, the scale of geography can be a key issue since majority of mesoscale predictions have a horizontal span of about $5 \mathrm{~km}$. For a distribution system, this scale may be too large. Other complications involved are availability of the data.

Figure 1.2 shows a plot of 5-minute average values of wind speeds on a randomly chosen day in the year 2009 in Elizabeth City, NC. The importance of predicting the power output of wind along and complexities involved in short-term prediction are evident from this plot. Variations in wind speeds imply fluctuations in the output of the turbine.It is easy to visualize the importance of short-term predictions from here. 


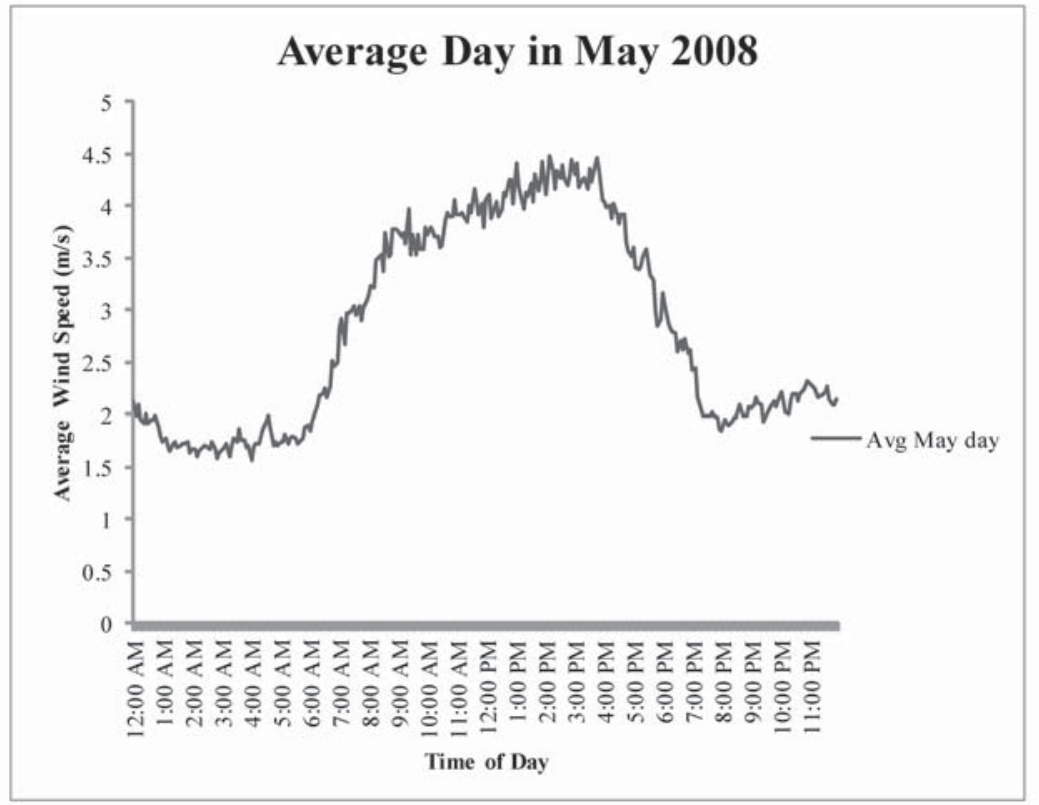

Figure 1.3 - Wind Speeds on a Monthly Average

Figure 1.3 shows how fluctuations are smoothed out over a monthly average. From plotting average data over different periods of time it is quite evident that the longer the time period, the smoother the average wind speed curve will be for a given location. Hence, it can be inferred that calculating an average power output over a longer time period can be fairly accurate given that we have large quantities of data. This fact gives further significance to the problem of a short term prediction. It is on the short term basis where the wind speeds have a large variance and hence good prediction methods become important. Different methods of prediction are summarized later in Chapter 2.

2. Distribution systems are different from the generation and transmission system counterparts due to their radial topology and unbalanced load flow. Among all the levels of power systems, from bulk transmission to end-user consumption, distribution systems are the elements where the impact of Smart Grid technologies and their implementation will be substantial. However, this transformation requires an establishment of effective computational ability, reliable communication in- 
frastructure and rapid restoration techniques in case of system anomalies.

3. An important fact that was observed was that mathematical models for smaller distribution systems need not be computationally expensive and hence simple solutions such as customized look-up tables need to be developed for switching operation.

\subsection{Research Contributions}

The following are the main contributions of this thesis:

1. First goal is to calculate probabilities of various wind speeds. Two different techniques to calculate probabilities of wind speeds are employed using historical data. For parametric approach, Probability Density Functions (PDFs) are obtained by calculating parameters from the available data set. The PDF expressions are then used to calculate the probability of each wind speed and power output is predicted. Using the same historical data analysis, nonparametric probability calculations are also carried out. Results from both the techniques are compared.

2. After calculating and comparing probabilities of various wind speeds, power corresponding to each wind speed is calculated. Probabilities calculated earlier are used to calculate average power from wind.

3. A small 29-node distribution system is developed to enumerate possible the switching actions in presence of wind generation. The calculated power output is used as an output of the wind generation present in this system. Feasibility constraints are defined and applied in order to identify the feasible combinations under various operating conditions.

\subsection{The Remainder of the Thesis}

The remainder of this thesis consists of a background on modern distribution systems and distributed generation, effects of distributed generation on distribution system at 
both primary and secondary levels, stochastic modeling of wind speed and solar irradiance along with the data used for building and testing the model. The last part discusses the results obtained followed by an analysis of the results and contributions made by this work.

- Chapter 2 conceptualizes the methodological development of this research in wind generation and state-of-the-art wind farm SCADA systems and operations.

- Chapter 3 elaborates on statistical analysis of historical wind speed data. Parametric and nonparametric statistics are used to calculate probabilities of various wind speeds. Results from both the methods are compared.

- Chapter 4 explains calculation of power for all wind speeds. Probabilities calculated are incorporated in the calculated power.

- Chapter 5 discusses distribution system reconfiguration using a small test system. A 29-node test distribution system is developed to test the switching topologies under different operating conditions and different levels of outputs from wind generation.

- Chapter 6 is the synopsis of the results obtained along with research contributions made by this work. Possible scope for future work is discussed. 


\section{Chapter 2}

\section{Background and Literature Review}

The first section of this chapter will discuss about different state-of-the-art methods used for predicting speed at different scales, followed by power in wind and influential factors therein. Further, distribution system topologies and reconfiguration be discussed, followed by a review of scholarly work in the area of distribution system emergency conditions and restoration operations.

\subsection{Wind Speed Prediction}

Wind speed prediction forms basis for calculating anticipated power production from wind turbines. Sufficiently accurate wind speed prediction is crucial at both planning and operating stages. The fuel insensitivity of wind is of no use without exhaustive wind resource evaluation at a site on planning stage. Once the wind site has been commissioned, it is of interest to know the anticipated power output from the facility for operational purposes.

\subsubsection{Scales of Prediction}

Wind speed predictions are conducted at different time scales for a variety of purposes. Figure 2.1 shows different scales of prediction and their integration. Listed below are the definitions of different time scales adopted here: 


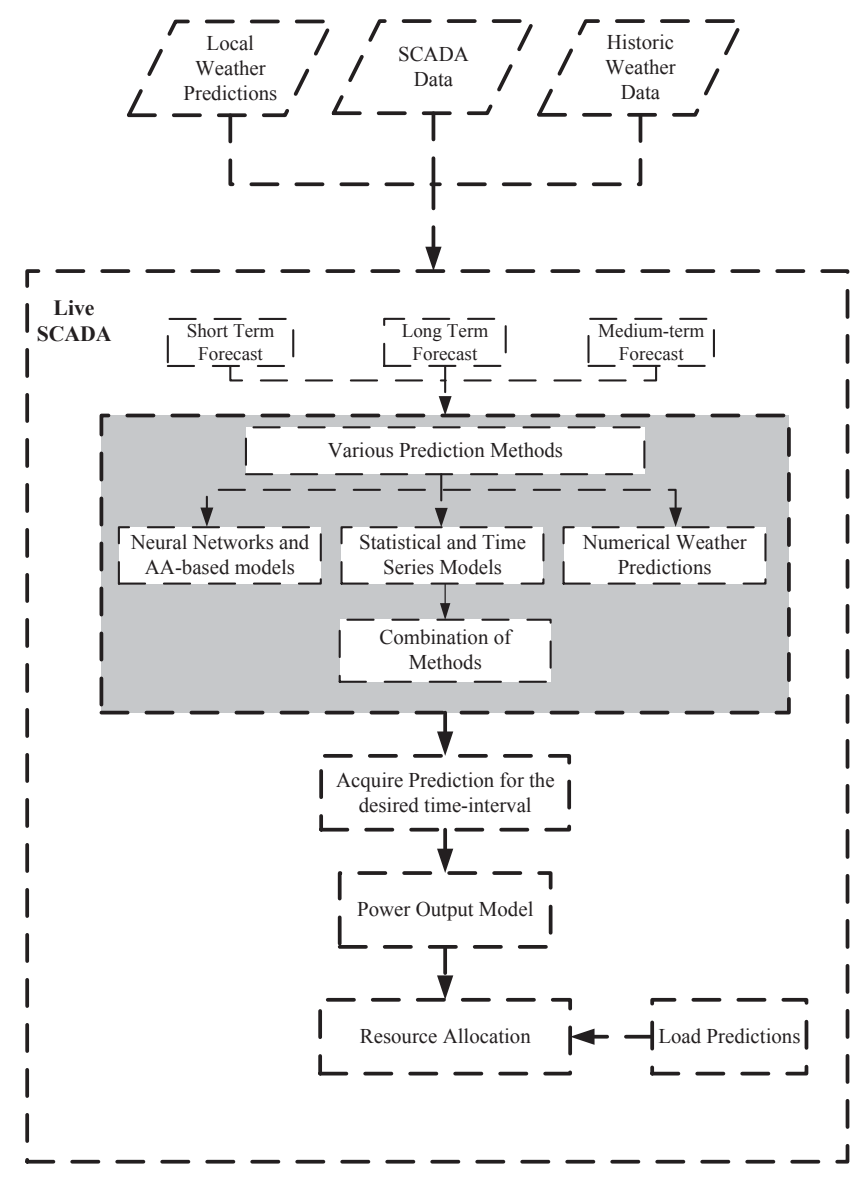

Figure 2.1 - Wind Power Forecasting Model

1. Long-Term Predictions: Long-term predictions are of the time scale of several months to up to few years. They are extremely important on the planning stage.

2. Medium-Term Predictions: Medium-term predictions range from several days to several months. These are important for trade purposes. Utilities and investors can gain from anticipating an amount output from the DG facilities ahead of time in order to validate green energy agreements and so on.

3. Short-Term Predictions: Short-term predictions are of the order of several minutes to several hours. They are extremely crucial for operational stages. Having better short-term predictions also improves reliability of the system. 


\subsubsection{Numerical Weather Predictions}

Numerical Weather Predictions (NWP) models are three-dimensional models based laws of thermodynamics and, conservation of momentum and energy. Partial differential equations of various degrees are developed and solved for on each point of the three dimensional grid. Accuracy of each model depends upon the equations used in the models and their initial conditions. NWP methods have the highest accuracy among all the techniques. Some examples are High Resolution Limited Area Model (HiRLAM) [10], Mesoscale Model - Version 5 (MM5) [11], Global forecasting System (GFS) [12] and Weather Research and Forecasting (WRF) [13]. Methods and tools employing NWP are utilized for meteorological purposes. The geographic scale of NWP is large and this may prevent their utility in small-scale electrical power distribution systems.

\subsubsection{Statistical Models}

Statistical models utilize historical data and patterns observed in past in order to calculate the probability of a certain event, in this case, a wind speed. Statistical models can be further divided into parametric and nonparametric models.

Parametric models [14] assume an underlying probability distribution and define relevant parameters for that distribution. These parameters are then calculated based on the historical data and used to calculate probabilities of various events. Some of the concepts used are those of Random Variables (RV) and their functions, Series of RVs and regression models.

Nonparametric models [15] are purely data-centric and do not have any underlying assumptions about the variable in question and hence are more generic. Results obtained by parametric stochastics can be achieved by nonparametric stochastics with more rigorous analysis. Accuracy of these is highly dependent on the quality and amount of data available.

\section{Direction of Wind}

Another important stochastic aspect of wind not included in the discussion above is the direction of the wind [16]. Direction of the wind affects the angle of attack on the 
Table 2.1

Wind Speed Prediction Techniques

\begin{tabular}{lll}
\hline Prediction Method & Features & Examples \\
\hline \multirow{2}{*}{ Numerical Weather Prediction } & Uses thermodynamics and 3-D grids & HiRLAM [10] \\
& Very large geographic scale & MM5 [11] \\
& For predictions of shorter terms & GFS [12] \\
\hline \multirow{3}{*}{ Statistical Methods } & Uses parametric and nonparametric Statistics & ARMA [23] \\
& Time series models for short term predictions & ARIMA \\
& $\begin{array}{l}\text { Probability Density Functions (PDFs) for long- } \\
\text { and medium- term predictions }\end{array}$ & \\
& $\begin{array}{l}\text { Accuracy depends on resolution of historical } \\
\text { data for the geographic location }\end{array}$ & \\
& &
\end{tabular}

blade [17], hence significantly affecting the power output. Analyzing wind direction is done with circular verions of Gaussian probability distribution, which take into account the angular nature of wind speed as random variable. The data set collected here does not provide information about direction of the wind and hence this analysis is beyond the scope of the thesis. It should be noted that most of the wind turbines are equipped with directional control in both the nacelle and the blades.

\subsubsection{Summary of Methods}

Several alternatives to predict wind speed on various time-scales have been presented. For long and medium term analysis, statistical methods used to calculate probabilities of wind speeds at a given location give good results. NWP methods are useful for short term predictions. Time-series approach of statistical methods that uses auto-regression moving averages, is also a useful way of short and medium term wind speed forecast.

Different combinations of methods for prediction have been implemented with various degrees of success [18], [19]. Predictions using Neural Networks [20], [21] are reputable, but they must be trained heavily in order to efficiently predict the wind speeds. Various existing methods [22] for wind speed predictions are listed in Table 2.1. 


\subsection{Wind Power}

Power produced by wind generation depends on several different factors, wind speed being the most influential among all of them. Several methods have been developed to correctly model and predict the wind speed at each time scale [24]. Wind power prediction softwares [25], [26] use the predicted wind speed values as one of the inputs.

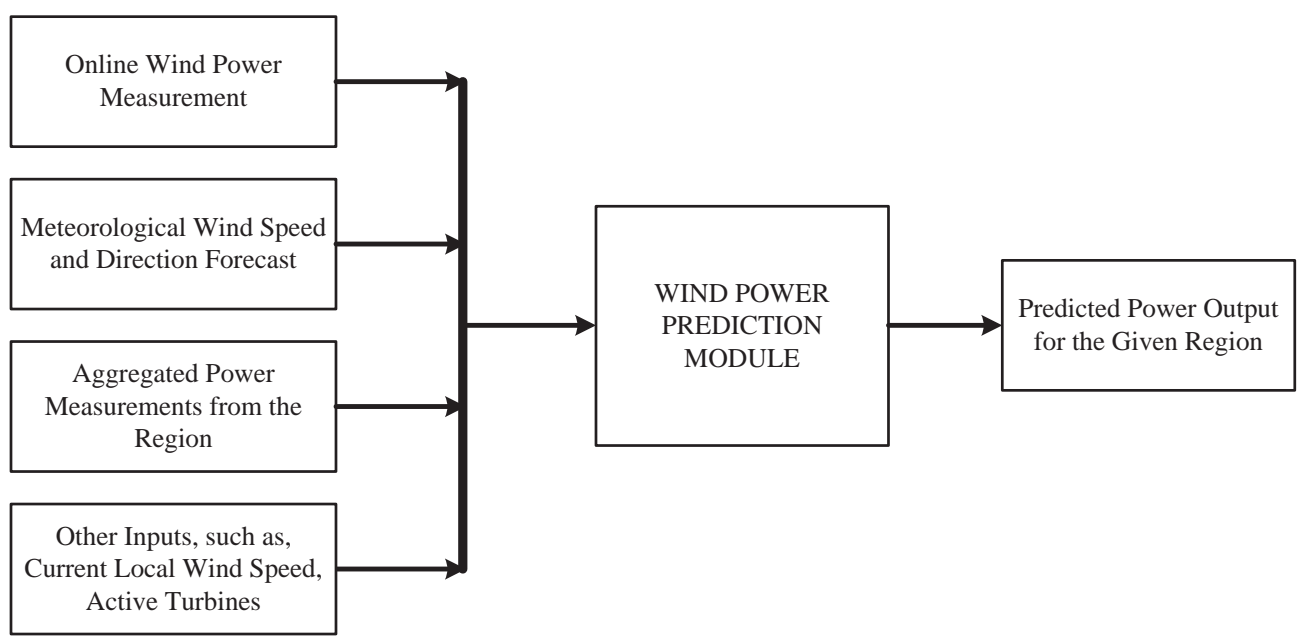

Figure 2.2 - Wind Power Prediction

Figure 2.2 shows a combination of various prediction techniques used for predicting power output from wind generation. Before delving into the prediction techniques, the equations governing the power contained in wind are discussed. An upper limit imposed on the maximum power effectively captured is also reviewed. Energy conversion taking place in wind turbines is from kinetic energy to mechanical energy and subsequently, electrical energy. Each of these conversions has some amount of loss. It is, however, important to know the amount of useful power that can be extracted from wind. Total power contained in wind can be given by the following expression:

$$
P=\frac{1}{2} \rho A V^{3}
$$

where, $P=$ Power contained in wind $(\mathrm{W})$, 
$\rho=$ Air density $\left(\mathrm{kgm}^{-3}\right)$,

$A=$ Swept area $\left(\mathrm{m}^{2}\right)$, and

$V=$ Wind speed $(\mathrm{m} / \mathrm{s})$.

Equation (2.1) gives the total kinetic energy energy contained in wind at a given velocity for a given cross-sectional area. It is clear from the expression that for a twofold increase in wind speed, the increase in power is eight-fold. The Equation (2.1), however, is far from the actual amount of energy captured. The energy contained in wind is dependent on air density, swept area of the blade and velocity, however, the energy captured by a turbine depends on the aerodynamic design of the blades. Power captured by each turbine depends on Lift Coefficient $C_{L}$, Drag Coefficient $C_{D}$ along with tip-speed ratio $\lambda$, and as a result, Coefficient of performance, $C_{P}$ for each machine is defined. $C_{P}$ gives a measure of the power actually captured by a turbine. The value of $C_{P}$ ranges from 0.25 to 0.45 in practice. However, there is an absolute theoretical limit for the amount of power that can be extracted from wind. This limit is known as Betz limit.

\subsubsection{Coefficient of Performance and Betz Limit}

The Equation (2.1) gives the total power contained in the wind, which essentially does not translate to the total power captured by the given machine [17]. Actual power captured by a given machine is given by:

$$
P_{\text {wind }}=\frac{1}{2} \rho A V^{3} C_{P}
$$

where,

$C_{P}=$ Coefficient of performance of a given turbine.

Coefficient of performance, $C_{P}$ of any power generation unit is given by the ratio of Total Power Extracted From Wind and Total Power Contained in Wind. $C_{P}$, as expected, is unique for each generating unit. There is a theoretical upper bound on $C_{P}$, known as Betz limit, which is the maximum power that can be extracted from wind with zero losses. This bound stems from the fact that mass rate flow, given by $\rho A V$ for any fluid remains constant under ideal conditions. 


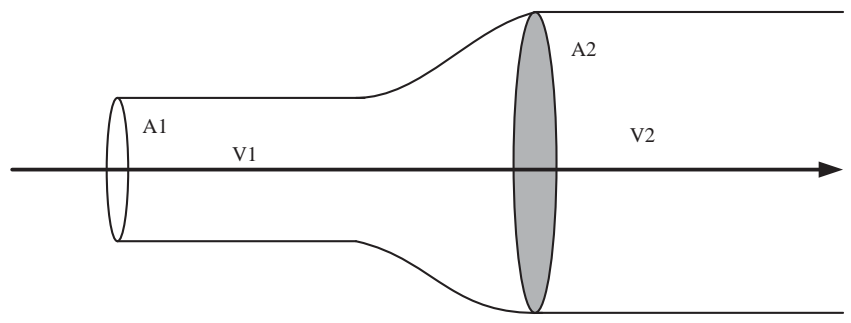

Figure 2.3 - Mass Rate Flow

As shown in Figure 2.3, for a larger cross-sectional area, velocity of the flow will reduce and vice versa. This can also be thought of as when wind goes through a turbine, if all of the power from wind was absorbed, the energy in the wind will have to fall to zero but this does not happen and even under ideal conditions. With proper mathematical manipulations, the upper bound to the energy absorbed can be found to be $\frac{16}{27}$. This means that a theoretical maximum of only $59.25 \%$ of the total power contained in the wind can be captured. Hence, the maximum power that can be extracted from wind [17] is given by Equation (2.2). To conclude, the power in wind depends heavily upon the wind speed and is, hence, highly intermittent.

\section{Air Density}

Air density at a given location is a function of air temperature, air pressure and the altitude at which it is being measured and is given by the following expression [17]:

$$
\rho(z)=\frac{\rho_{0}}{R T} \exp \left(\frac{-g z}{R T}\right)
$$

where,

$\rho_{0}=$ Standard air density at sea level $\left(\mathrm{kgm}^{-3}\right)$,

$z=$ Altitude $(\mathrm{m})$,

$R=$ Gas Constant $\left(287.05 \mathrm{~J} \mathrm{~kg}^{-1} \mathrm{~K}^{-1}\right)$, 
$T=$ Air temperature $(\mathrm{K})$, and

$g=$ Gravity constant $\left(9.81 \mathrm{~m} / \mathrm{s}^{2}\right)$.

Air density does not vary significantly and hence will be considered a constant here, standard air density at sea level being $1.225 \mathrm{kgm}^{-3}$.

\subsection{Integration of Wind Generation}

With increasing penetration of wind energy into the distribution system, a need to make more improvements in the well-established load flow techniques has risen [27]. Load flow analysis methods have been proposed for the active distribution systems and microgrids [28]. This method is applicable for both balanced and unbalanced load flows.

While planning issues such as optimal placement and penetration of DG [29] remain of interest to the research community, experiences of implementation of DG have also been documented [30], [31]. With increasing field experience, more challenges come forward on operational front and operational aspect of including DG, especially of intermittent nature, remains somewhat of a less chartered territory.

\subsubsection{Wind Farm SCADA}

A Supervisory Control and Data Acquisition (SCADA) system is a centralized control and monitoring system that receives data from various transmitting nodes known as Remote Terminal Units (RTU). Wind SCADA systems, usually provided by the turbine manufacturer, are the nerve centers for wind farms. The primary function is to monitor and control individual turbines, wind farm as a whole and other system parameters. Central server of SCADA communicates with remote units deployed at each wind turbine mostly using fiber optic cables [32]. Types of data monitored by SCADA (see figure 2.4) can be catagorized into the following: 


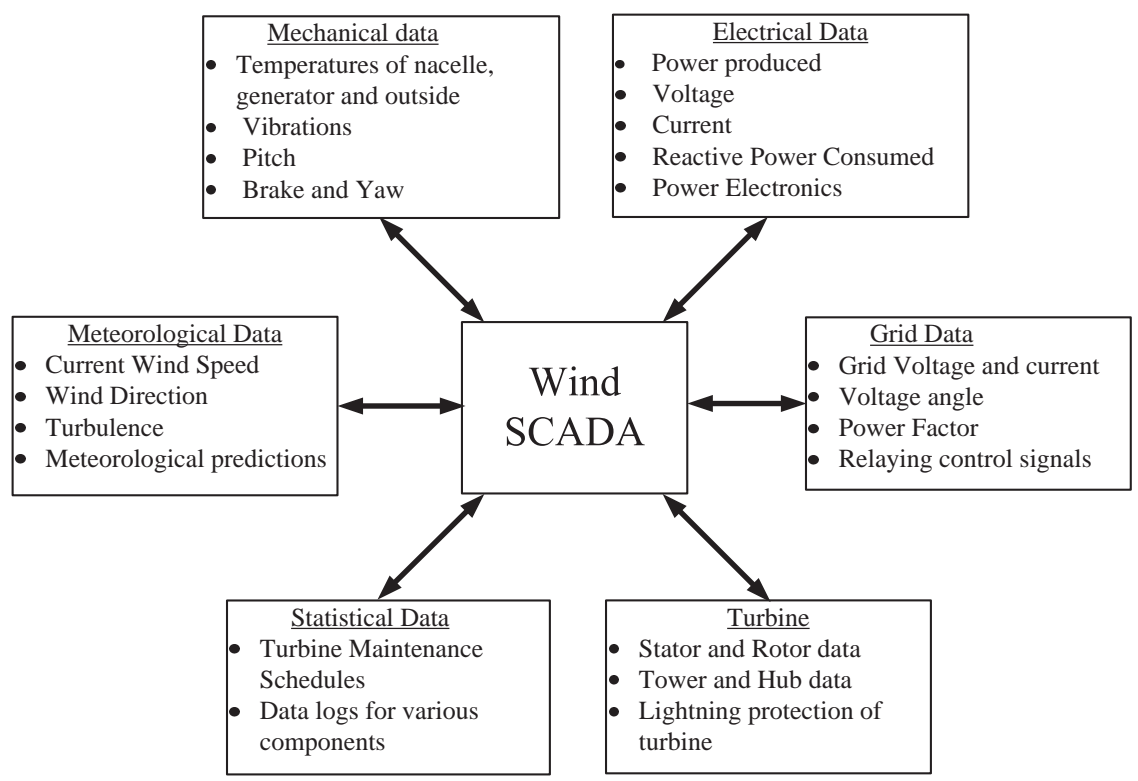

Figure 2.4 - Wind SCADA

- Electrical Data: Electrical data includes power produced by the turbine, voltage, current, power factor and reactive power consumed.

- Mechanical Data: Mechanical data includes temperatures of generator, nacelle and the surroundings, vibrations, pitch, gearbox, oil-levels, etc.

- Grid Data: This is the reference data obtained from the grid. It includes values of various electrical quantities mentioned above from the grid.

- Meteorological Data: This includes current weather conditions, wind direction and speed, turbulence as well as predictions.

- Statistical Data: This includes data logs, maintenance schedules of turbines, etc.

- Turbine Data: This is the individual turbine data. It includes the miscellaneous data that is not included in the five categories discussed above.

With a reliability-centered monitoring [33], data obtained from different system components can be used to detect different types of mechanical or electrical failures. Most of the SCADA systems are supplied by their respective manufacturer and often lack flexibility of operation [34], [35] to comply with the farm owners and/or operators. As 
a result, SCADA system from the grid, then, may have to compensate for the apparent lack of flexibility of wind SCADA.

\subsection{Distribution System Topologies}

A notable aspect of this thesis is the focus on distribution systems, since distribution system are likely to have the highest impact from the Smart Grid initiative [1]. The emphasis is also due to the following peculiarities of distribution systems as opposed to transmission systems, which change the dynamics significantly:

1. Radial Topology: Distribution systems terminate the electrical power system into loads where the electricity is consumed [36]. It is only logical to have a simplified topology for economical purposes. Protection-coordination of the system mainly consists of fuses and reclosers and seldom has a directional sensitivity [37]. Hence, it is convenient to have a radial system from this perspective, as well.

2. Unbalanced Loads: Majority of residential and commercial loads are served as single- or two-phase loads. This evidently results into an unbalanced system. The basic assumption of a three-phase, balanced system employed in loadflow studies yields erroneous results in distribution systems and hence a phase-by-phase analysis is crucial for reliable loadflow studies.

3. Line Models: Shorter lengths of distribution lines and a high $R / X$ ratio implies that the resistance of the lines can no longer be neglected [38], nullifying another assumption of circuits being purely reactive in transmission lines. This brings an added level of complexities in analysis.

4. Frequent Changes in Topology: A known system topology with a set of important parameters is crucial for analysis. There is often lack of up-to-date information of the distribution system topology [38]. Also, it is easier and more likely to alter the distribution system topology. This may result into an inexact representation of distribution system under scrutiny. 
For this work, distribution systems are divided into two parts: Primary and secondary distribution systems. They are normally medium and low level $(12.47 \mathrm{Y} / 7.2 \mathrm{kV}$ or $24.9 \mathrm{Y} / 14.4 \mathrm{kV}$ [36]) distribution systems. Differences between primary and secondary system are noted below.

When analyzing a distribution systems, it is worth noticing that distribution systems are often unbalanced, whereas, transmission systems are balanced and three phase. The equivalent single phase representation of transmission network is valid due to its balanced nature. This, however, is not true for distribution systems due to their unbalanced nature. All the components need to be modeled in more detail and for individual phases since single-phase equivalents are not valid.

\subsubsection{Primary Distribution System}

The part of the distribution system from the point where transmission system terminates into a distribution substation to distribution transformers is regarded as the primary distribution system. This network is made up of main feeders and is generally lightly meshed in order to increase the reliability of the system. Tie switches are used to allow different configurations in order to minimize the out of service part in case of an emergency. 


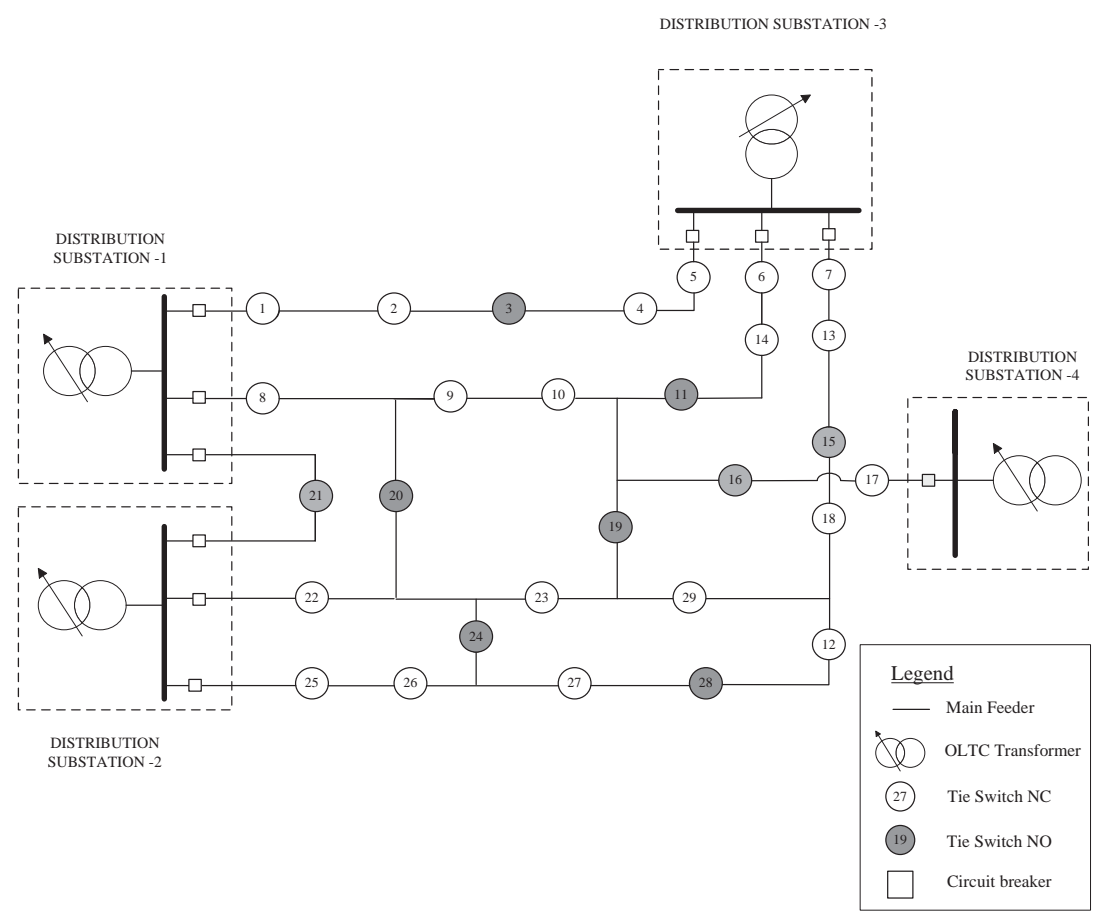

Figure 2.5 - Primary Distribution System

Figure 2.5 shows the topology of a primary distribution system. Voltage levels for primary distribution system are typically medium level voltages that have ranges in the United States. Feeders branch out into distribution transformers and eventually subfeeders and laterals. To summarize, the features of primary systems are:

- normally radial or lightly meshed,

- medium voltage levels of up to $69 \mathrm{kV}$,

- normally three-phase and balanced, and

- sophisticated protection schemes consisting of circuit breakers at distribution substation end and reclosers and coordinated fuses on the feeders. 


\subsubsection{Secondary Distribution System}

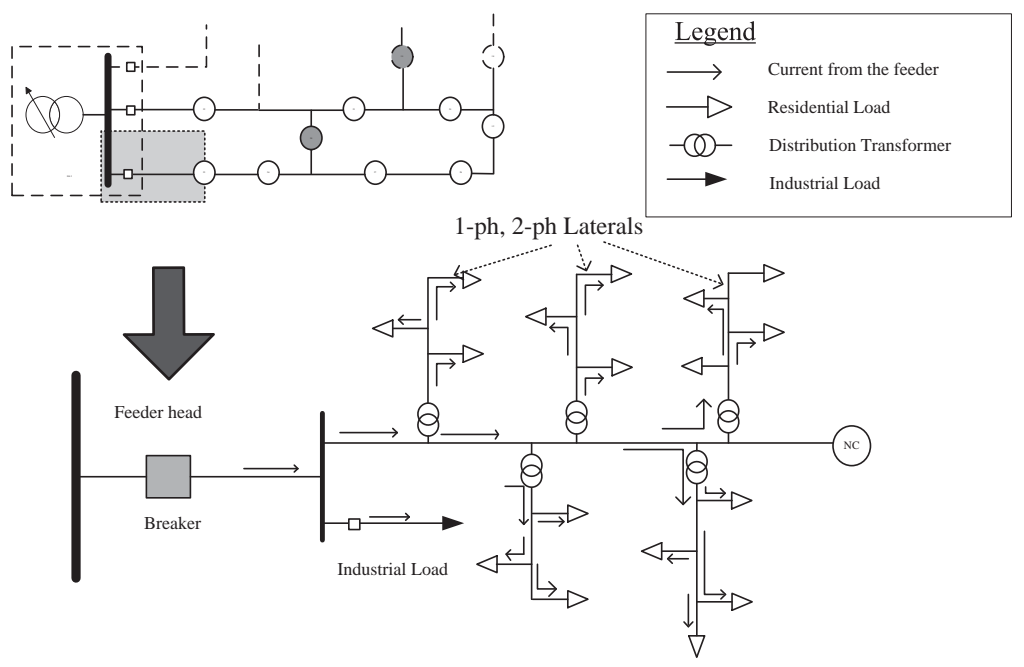

Figure 2.6 - Secondary Distribution System

Everything beyond distribution transformers is considered a secondary distribution system for this work. Secondary systems almost always have a radial topology. Secondary distribution systems are inherently unbalanced in nature. The loads are 1-, 2- or 3-phase, grounded or ungrounded. In order to accurately model the behavior of a distribution system, single phase equivalents do not suffice. Each of the components such as, lines, loads, distribution transformers and voltage regulators must be individually modeled for all three phases separately.

Figure 2.6 shows a zoomed-in version of one feeder. Feeders and main cables bifurcate into subfeeders, laterals and sublaterals via distribution transformers (DTs). DTs have a very important function of bringing the voltage down to usable limits for residential and commercial sectors at voltage levels of 120/240V. Laterals and sublaterals have variable number of phases depending upon the type of the connected load. These are the terminal points for the electricity and it is thereby consumed. Protecting devices in the secondary systems are coordinated fuses. To summarize, features of secondary distribution systems are:

- mostly radial nature, 
- low voltage levels,

- loads can be1-, 2- or 3- phase, and

- protection schemes that consist of coordinated fuses.

\subsection{Distribution System Reconfiguration}

Distribution system topologies are subject to change frequently [38]. Transmission networks have looped topologies, are highly interconnected and are less susceptible to frequent topological changes. Distribution systems, on the other hand, are conventionally radial and owing to the autonomy of the consumer, are susceptible to frequent topological changes. Deliberate change in topology is achieved by provision of remote controllable switches. It is, therefore, of interest to learn about the motivation of deliberate topological changes and components that provide these changes.

\subsubsection{Remote Controllable Switches}

Provision of remote controllable switches referred to as tie and sectionalizing switches is made in distribution systems to provide alternative paths for the current to flow in order to serve the load. Thus, the main function of tie switches and sectionalizing switches is to facilitate system reconfiguration [39] under both normal and emergency operating conditions.

Tie switches are normally open and sectionalizing switches are normally closed. Reconfiguration of distribution system can, thus, be obtained by altering the state of these tie and sectionalizing switches as required.

Figure 2.5 shows an example distribution system with tie and sectionalizing switches located throughout the system. Depending upon the size of network and number of such switches, several alternative paths can be provided for serving the load.

\subsubsection{Motivation for Reconfiguration}

The prime goal during the planning stage in a distribution system is to reduce construction costs, however, system losses and performance indices determine feasible econom- 
ical operation in the operating stage. Tie and sectionalizing switches provide means for altering system topology under different conditions. The goal is to study the effects of incorporating wind generation on system operations in presence of tie and sectionalizing switches.

Inclusion of wind generation introduces complexity in these switching actions. Involvement of generating facility close to the load in form of wind generation may appear to reduce the losses by eliminating the need for transmission over long distances, but this is not always the case. Moreover, addition of wind generation, as in case of loss reduction, plausibly helps improve the voltage profile, however, under certain conditions, contrary is true [40]. The following are the motivations to alter the distribution system topologies:

\section{Under Normal Conditions}

1. Loss Reduction: Minimization of losses is the most important constraint after serving all of the load satisfactorily. Distribution system losses can be given by:

$$
P_{\text {LOSS }}=I^{2}(R \cos \theta+X \sin \theta)
$$

Losses depend upon the length of lines, types of conductors and load characteristics. Higher losses translate to lower profits for utility and hence, minimizing losses becomes very important. When there are alternative paths available to supply the load, the path with the lowest should be chosen. Provision of remote controllable switches can facilitate this.

2. Improvement of Voltage Profile: Voltage drop along the feeder is usually given by:

$$
V D=I(R \cos \theta+X \sin \theta)
$$

Voltage support is usually provided by strategically placed capacitor banks. Switching combinations can be helpful in improving voltage profile by efficiently supplying the load using the path with least voltage drop. 


\section{Under Emergency Conditions}

1. Fault Isolation: Under fault conditions, the faulty part of the system must be isolated immediately from the healthy part of the system. In absence of redundancy, this may mean disconnecting more number of customers then necessary. Sectionalizing switches allow minimization of the part of the system affected by a fault, thus decreasing the number of customers interrupted.

2. Improvement of Reliability: Distribution system reliability is measured in terms of various indices, System Average Interruption Duration Index (SAIDI) and System Average Interruption Frequency Index (SAIFI) are two important indices. These indices are given by [36]:

$$
\begin{gathered}
S A I D I=\frac{\sum r_{i} N_{i}}{N_{T}} \\
\text { SAIFI }=\frac{N_{i}}{N_{T}}
\end{gathered}
$$

where,

$N_{i}=$ Number of customers affected,

$N_{T}=$ Total number of customers served, and

$r_{i}=$ Restoration time for each fault.

By proper operation of tie and sectionalizing switches, number of affected customers $N_{i}$ under fault conditions can be reduced [41], thus improving the performance indices. Performance indices also depend on other factors including efficient fault location algorithms, proper coordination of protection equipment and rapid restoration techniques as well. Detailed discussion of these aspects, however, is beyond the scope of this work.

The number of possible alternative paths increases with increasing number of switches. Various optimization techniques [42] employing heuristic approaches [41], [43] have been developed for sizable networks with a large number of switches. These promising approaches, however, can be computationally expensive. For a smaller system, switching combinations under various system conditions can be simply enumerated for ease of operation. The goal is to determine all possible topologies and their feasibility beforehand in order to reduce the number of all possible topologies to ones that are actually 
feasible. Of course, the feasibility here is based on certain conditions, which will be discussed in the next section.

It is only logical to enumerate the number of scenarios in smaller distribution systems, where number of possible combinations is not memory-expensive due to a smaller number of DG. In order to accomplish this, first step would be to define and apply constraints: the set of constraints applied have to start with the unavoidable basic constraints. The switching combinations that do not fulfill these basic constraints can be simply discarded. Further constraints can be applied to the remaining combinations for optimal operation. Detailed discussion of the feasibility constraints is in Section 5.2.2.

\subsubsection{Fault Location, Isolation, and Restoration}

Knowledge of fault location expedites system reconfiguration, hence, it becomes important to list important contributions in this area. An algorithm has recently been developed that goes through the circuit to identify the locations that match the fault current measured at the substation and then using a probabilistic modeling, also tries to predict the location of the fault [44]. Method based on the bus impedance (Zbus) matrix, uses measurements obtained at the substation for fault location in overhead distribution lines. Two different types of approaches are developed for balanced and unbalanced non-radial and radial systems [45].

Faults in underground distribution lines are harder to locate and restore. Numerical modeling of incipient faults in underground systems using field-recorded online voltage and current measurements has also been proposed [46]. A fuzzy CE-Nets technique has been developed and a new reasoning for fault diagnosis in distribution substations is presented. The proposed approach claims to be capable of representing uncertain knowledge and performing fuzzy reasoning through matrix based transformation [47] A possible way of mitigating cascading failures in case of major black outs has also been proposed for large systems. A new machine learning approach for protective relays based on binary hypothesis testing, Support Vector Machines (SVMs), and communications between the protective relays and the Supervisory Control And Data Acquisition (SCADA) has been developed [48]. 


\subsection{Summary}

The aim of this chapter was to provide necessary background to the reader. The following were discussed in detail here:

1. Importance of wind speed prediction at different time scales and techniques used for prediction at these levels was discussed.

2. Power contained in wind and the amount of power extracted were discussed. The theoretical limits on the amount of power extracted were noted.

3. Distribution system topologies were identified and classified in primary and secondary distribution systems.

4. Motivation and components responsible for distribution system reconfiguration were also discussed.

5. The content was concluded by a short discussion on state of the art fault location, isolation, and restoration techniques. 


\section{Chapter 3}

\section{Prediction of Wind Speed}

This chapter discusses the wind speed forecast techniques used and relevant simulations. State-of-the-art mathematical models and techniques have been summarized in Chapter 2. The more complex NWP and time series models are more suitable for large scale wind deployment, given the higher applicability and availability of resources. NWP methods are computationally expensive and may be redundant in small distribution systems. The primary goal of forecast in smaller systems is to be able to anticipate the amount of power generation from wind and operate the system more efficiently under normal as well as emergency conditions.

The primary goal of this thesis is to identify and suggest suitable switching combinations under different levels of output from wind generation in a distribution system. It should be emphasised that the goal here is not to make prediction of one particular wind speed. Rather it is our goal to calculate probabilities of each individual wind speed for a given hour in a given season. The time-frame under scrutiny is of one hour length. Knowledge of probabilities of different possible wind speeds, and thus, the relevant power output, prepares the system operator for various possible scenarios depending upon the anticipated output from the wind generation. In order to calculate probabilities, two approaches were compared, namely: Parametric and Nonparametric Probability calculations [14].

Figure 3.1 shows both the approach used for calculating probabilities of each wind speed for the given location. Historical data for wind speeds is used in order to calculate probabilities of each of the wind speeds observed in past. 


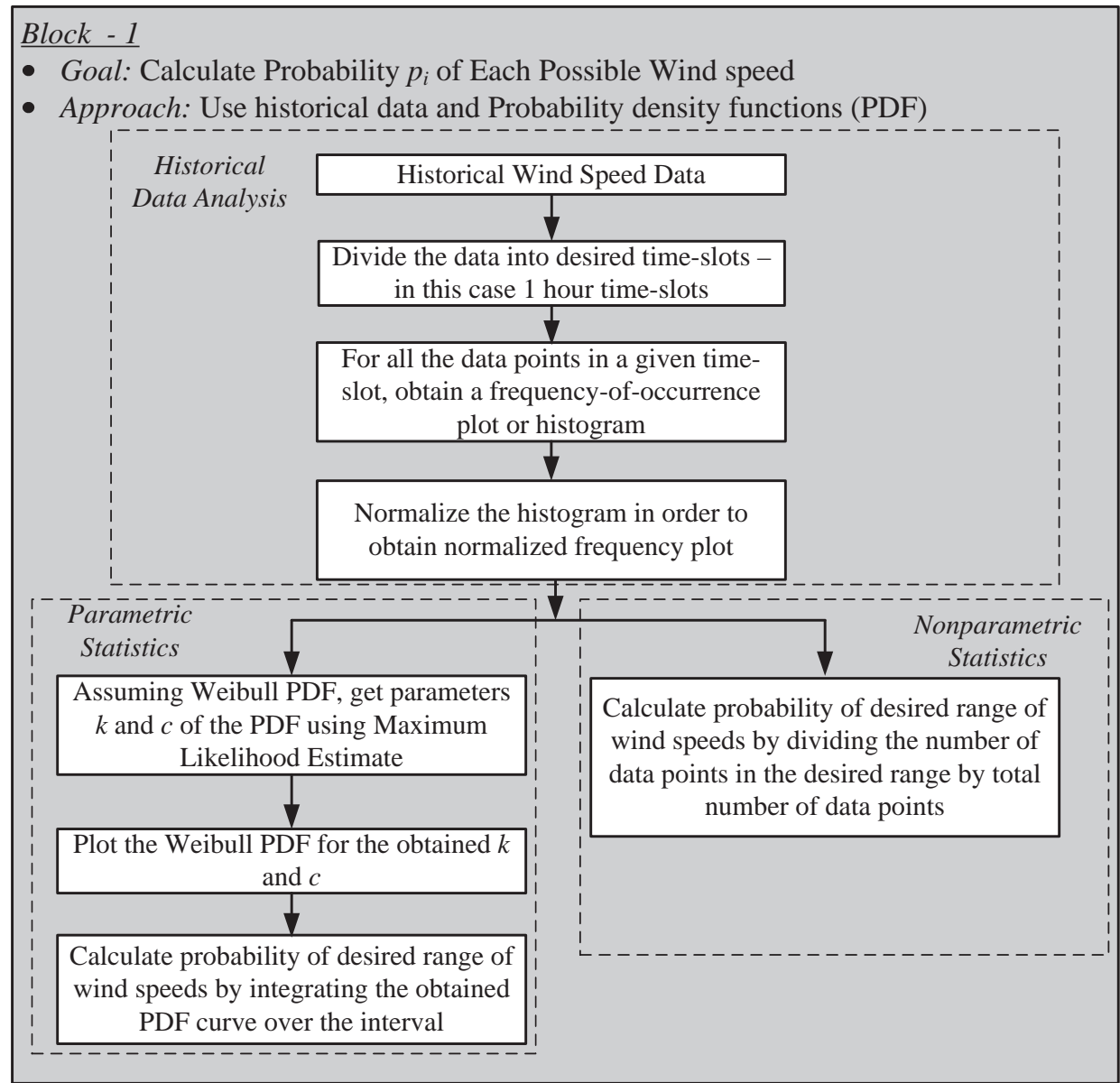

Figure 3.1 - Wind Speed Prediction Methodology

The rest of this chapter begins with historical data analysis. The subsequent part explains how to calculate probability assuming a probability distribution for the given set of data, followed by the part explaining the nonparametric approach to calculate probabilities. Simulation results for both approaches are compared in the final section of this chapter.

\subsection{Historical Data Analysis}

In order to maintain the integrity of the intermittency of wind, real historical data [49] is used. The data is from weather stations at Elizabeth City, NC. Alongside the purpose 
of a more accurate analysis of wind speeds, methodologies used here to calculate wind speed probabilities are purely data-driven, hence, detailed historical data is the first step towards making predictions.

Figure 3.2 shows the division of the wind speed data used. Four years (from year

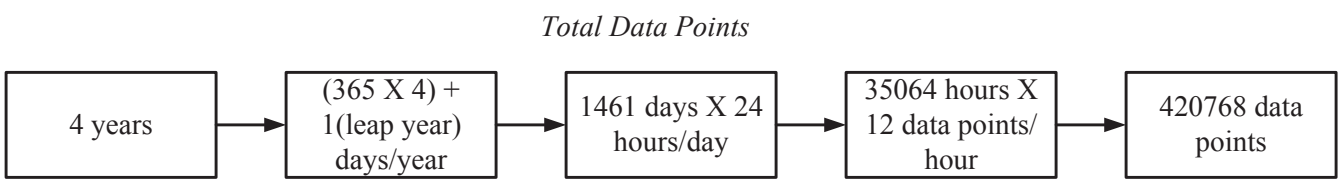

Figure 3.2 - Total Data Points

2007 to year 2010) worth of historical data from Elizabeth City, NC is used. This data has been made publicly available by the National Renewable Energy Laboratory (NREL) [49]. The reason to choose this particular site is the vast availability of detailed wind speed data. Data points are each a 5 minute average, collected over four years, results in a total of 420768 data points.

As seen from Figure 3.3 this data is further divided into four seasons: Summer, Fall, Winter, Spring. It is then divided into time frames of 1 hour each for 24 hours of a typical day in the given season. Each hour has 12 data points which are 5-minute average of wind speed. Data points belonging to a particular hour of the day for a given season are then condensed into bins of data, which consist of all of the wind speeds that occurred during the particular hour of day in that particular season. The reason to divide annual data into four parts according to seasons is to account for the seasonal changes in wind speeds. The following is the summary of historical data analysis:

- Historical data is the basis for the analysis performed here. Given that both the methods used are purely data-driven, calculation of probability is based on historical data.

- Simulating the wind speed data requires complex modeling, which is beyond the scope of this work.

- Data is divided into seasons and then time-of-the-day bins.

- Data points are divided such that each bin represents a particular time of day for a given season. 


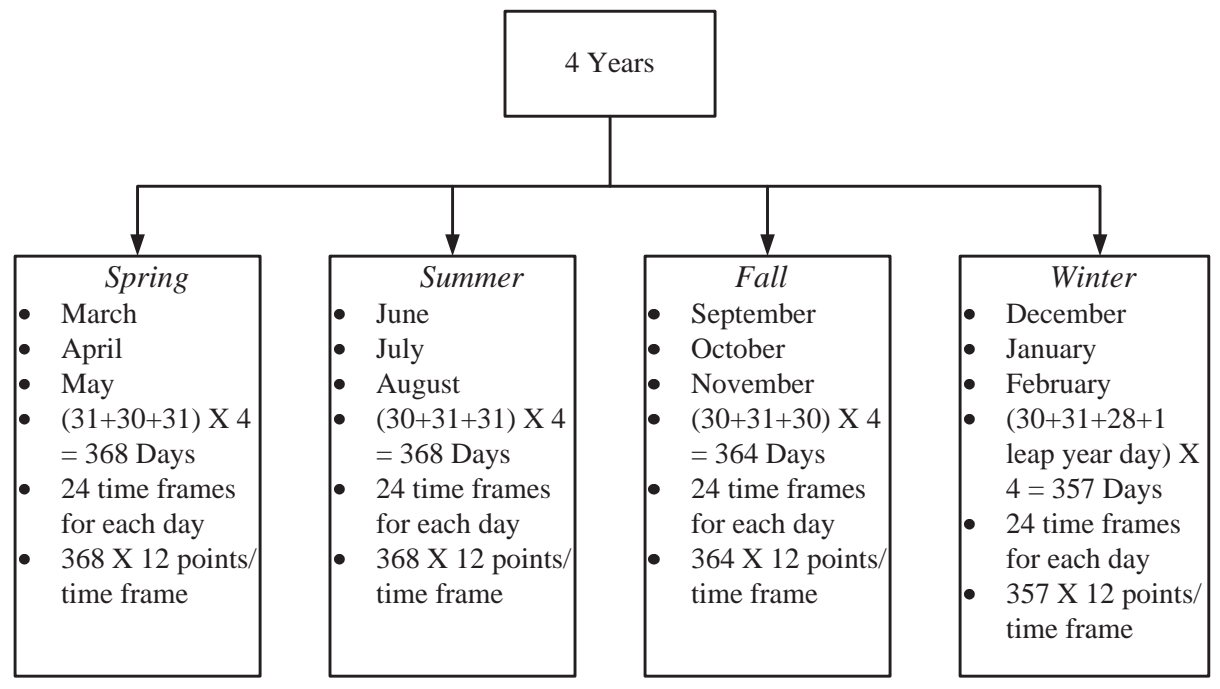

Figure 3.3 - Data Resolution

\subsubsection{Normalized Frequency Plots on Different Time-scales}

After sorting the data points within time-frames specified in Figure 3.3, frequency of occurrence plots of each wind speed in the said region are obtained.

$$
\text { Normalized Frequency }=\frac{\text { Number of times a wind speed is observed }}{\text { Total number of observations }}
$$

Figure 3.4 shows what a normalized frequency of wind speed as the variable of interest. This plot will also form basis for the nonparametric probability calculations. The distribution of data points as seen from Figure 3.4 in the frequency plots already resembles a Weibull density. The plots may also imply convergence of Maximum Likelihood Estimation (MLE) assuming a Weibull density for the given data set. However, there are two factors that heavily effect the visual and analytical proximity of the frequency plot to an actual Weibull density. The following is the discussion of these factors:

1. Number of Data Points: It must be noted that the amount of data influences the fit of the curve. The higher the number of data points, the more accurately the Probability Density Function (PDF) follows the frequency distribution of the data.

The first plot in Figure 3.5 shows the normalized frequency plot of a 5-minute 


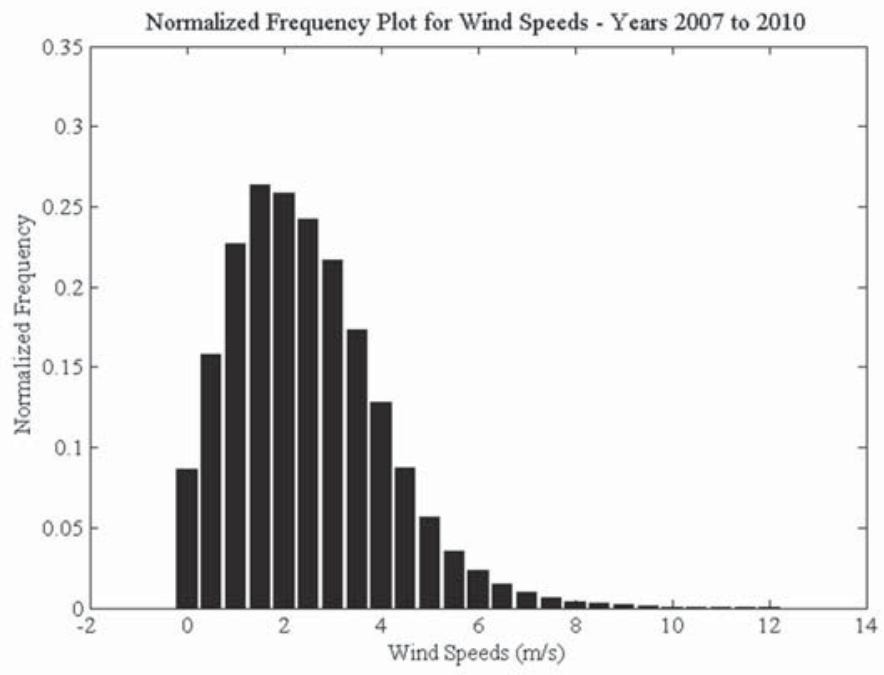

Figure 3.4 - Normalized Frequency Plot of Wind Speed Data

average wind speeds for the time frame of 2:10AM to 2:15AM in Winter, 2009 (90 data points). The second plot in Figure 3.5 shows a plot for all the wind speeds observed in year 2009 (105120 data points). This figure shows two extreme cases demonstrating an effect of total number of points considered for a frequency plot.

It is evident that for a smaller number of data points, the frequency distribution plot begins to lose its credibility. However, as the number of points increases, the probability density fits the data set better. This fact is also illustrated in the second plot of Figure 3.5. For data analysis here the number of data points for each time frame was around 4300 data points depending upon the number of days in a particular season.

2. Bin Size of Frequency Plots: Bin size is the size of each bar on the frequency plot. It is crucial to pick a suitable bin size in order to get the density curve to follow the frequency plot adequately. 

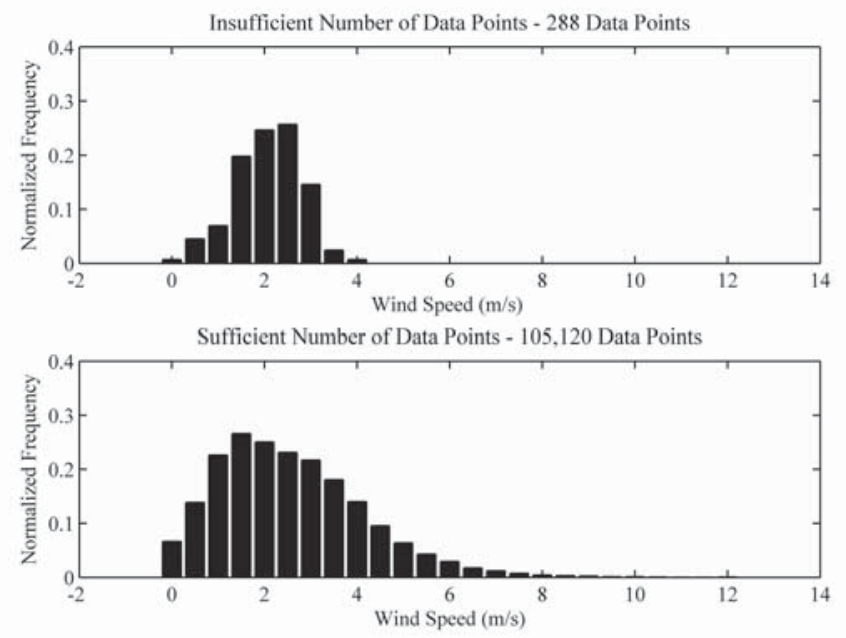

Figure 3.5 - Comparison of Number of Data Points
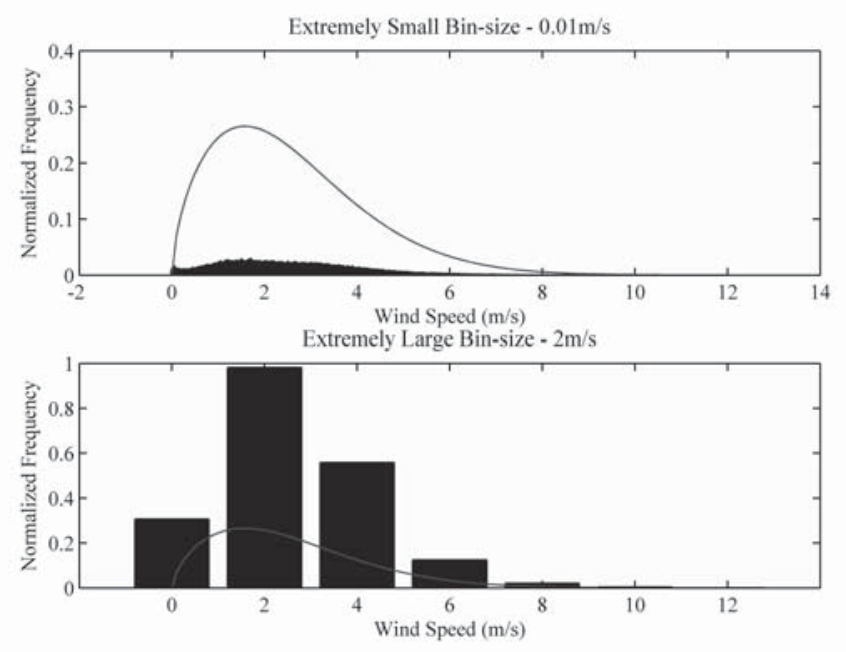

Figure 3.6 - Comparison of Extreme Bin Sizes

Figure 3.6 shows the impact of bin size. It is clear that for a much smaller step size of $0.1 \mathrm{~m} / \mathrm{s}$ on the top, data points are quite spread out. On the other hand, having a large bin size of $2 \mathrm{~m} / \mathrm{s}$ also does not produce satisfactory results. The default bin size chosen here is $0.5 \mathrm{~m} / \mathrm{s}$ (Figure 3.4 ), as it has satisfactory detail and the PDFs also follow it rather well. The same bin size will be used later to calculate probability from Cumulative Distribution Function (CDF) as well. 


\subsection{Probabilities Using Parametric Statistic}

Probability Density Functions (PDFs) are a reliable way of calculating probability of any observed wind speed, and hence, the power output of wind generation, given a sufficient amount of historical data. Given a large amount of historical data, reverse-engineering probability of a particular wind speed for a given time is of interest here. In order to proceed with the probability calculation using the parametric approach, it is essential to assume an underlying probability distribution for the variable under scrutiny. Weibull probability distribution was chosen for the following reasons:

- Weibull probability distribution offers versatility and flexibility that is helpful in describing the intermittency of wind speeds [50], [51].

- Accurate estimation of parameters $k$ and $c$ for Weibull probability distribution is easier compared to distributions such as Beta distribution.

- Wind speeds are known to follow Weibull density [17], [29], in some cases, probability distributions such as Rayleigh, which are simplified version of Weibull density.

- It was found after detailed analysis that the given data set also conforms to Weibull density, solidifying the assumption.

\section{Cumulative Distribution Function (CDF)}

A cumulative distribution function $(\mathrm{CDF}), F_{x}(X)$ of a random variable $(\mathrm{RV}) x$, (wind speed $v$, in this case) is nothing but probability $P_{x}$ of the RV $x$ being less then a certain value $X$. In our case, it is the probability of wind speed $v$ being smaller then a certain wind speed $V$ is the CDF of wind speed. Mathematically,

$$
F_{v}(V)=P(v \leq V)
$$

From the definition in Equation (3.2), it is clear that the CDF has values between 0 and 1. Properties of CDFs make it possible to calculate probability of a random variable, assuming a certaine probability distribution. 


\section{Probability Density Function (PDF)}

A Probability Density Function (PDF) of a random variable $x$, (wind speed $v$, in this case) is the derivative of cumulative distribution function.

$$
f_{v}(V)=\frac{d F_{v}(V)}{d v}
$$

In other words, from the definition of derivatives, PDF at a particular point gives the probability of a random variable being between two possible values. Since PDFs are derivations of CDFs, the area under any PDF curve is always 1 . This and other properties discussed below will form basis for probability calculation of any wind speed.

\section{Properties of PDFs and CDFs}

CDFs and PDFs have the following properties that are useful in calculating the probability of a certain wind speed [14]:

1. Given the PDF of a RV, CDF can be obtained by integrating the PDF expression:

$$
F_{v}(v)=\int_{-\infty}^{v} f_{v}(v) d v
$$

In case of Weibull probability densities, $\operatorname{PDF} f_{v}(V)$ is only valid for values greater then zero, which is intuitive, as the RV in question here is wind speed. Hence the expression in Equation (3.4) now becomes:

$$
F_{v}(v)=\int_{0}^{v} f_{v}(v) d v
$$

2. A useful property of $\mathrm{CDF}$ is:

$$
F_{v}\left(v_{1}\right)-F_{v}\left(v_{2}\right)=\int_{v_{1}}^{v_{2}} f_{v}(v) d v
$$

This property was utilized to calculate the probability of wind speed being between two values $v_{1}$ and $v_{2}$. This step size $v_{1}-v_{1}$ will be important in deciding the value of probability. 
3. From Equations (3.2) and (3.6) it is intuitive that:

$$
P_{v}\left(v_{1} \leq v \leq v_{2}\right)=F_{v}\left(v_{1}\right)-F_{v}\left(v_{2}\right)
$$

The properties above need to be mathematically manipulated in order to calculate probability from raw data numbers. Sections below discuss the calculation of probability of different wind speeds from historical data and relevant simulation results.

\section{Characteristics of Weibull PDF}

Wind speeds tend to follow Weibull probability density and mathematical expressions for Weibull density are discussed below. Weibull density is given by:

$$
f_{v}(v)=\left(\frac{k}{c}\right)\left(\frac{v}{c}\right)^{k-1} \exp \left[-\left(\frac{v}{c}\right)^{2}\right]
$$

where,

$v=$ Wind velocity,

$k=$ Shape parameter for Weibul density

$c=$ Scale parameter for Weibull density

Figure 3.7 shows possible shapes of Weibull density for different values of $k$ and $c$. The following is the description of three important parameters in Weibull PDFs.

1. Shape Parameter $k$ : Depending upon different values of $k$, the density takes on various shapes as displayed in Figure 3.7. Values of $k$ can simplify the Weibull density into a simple exponential density or take on other shapes as shown in Figure 3.7. It can also be referred to as the slope parameter, since essentially, it also affects the slop of the probability plot (not to be confused with PDF plot)

2. Scale Parameter c: As evident from Firgure 3.7, parameter $c$ is responsible for the scale of the density. Increasing the value of $c$ stretches the PDF farther out to the right, while reducing its peak. Similarly, decreasing its value pushes the PDF towards left, increasing the peak of it. 


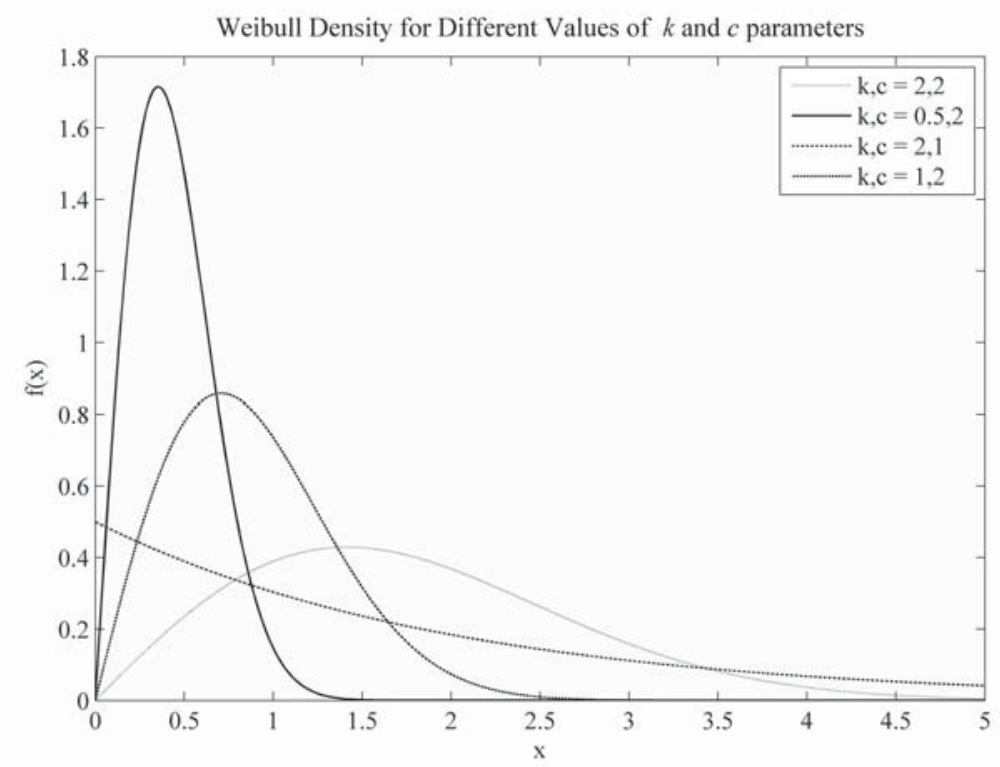

Figure 3.7 - Weibull PDF for different Values of $k$ and $c$

3. Location Parameter: A location parameter can also be included in some cases, which decides where on the horizontal axis the PDF is placed. This parameter, however, is not relevant here and will not be considered.

In order to be able to calculate probabilities of various wind speeds using Equation (3.8) discussed above, first step is to obtain these expressions from historic data available. Two parameters of interest in Weibull densities are $k$ and $c$. The following section will describe method used to calculate these parameters.

\subsubsection{Calculation of $k, c$ Parameters}

When solving for the parameters of the Weibull density function fitting the historical data set, iterative techniques must be used. Maximum Likelihood Estimation (MLE) is used to calculate parameters $k$ and $c$ from the data. Once the parameters are obtained, probabilities of all wind speeds observed are calculated by integrating the PDF expression (see Equation (3.7)). 


\section{Maximum Likelihood Estimation}

Maximum Likelihood Estimation (MLE) is a popular technique used to obtain parameters of a statistic model [14]. In MLE, an underlying probability distribution is assumed for the given set of observations or data. From the given set of data, MLE calculates parameters of underlying PDF such that the observed data has the highest probability of occurring, given the parameters obtained.

Let $x_{1}, x_{2}, x_{3}, \ldots$ be a set of data points. It starts by assuming a density $f$ that the given data set belongs to. Each probability density function has a set of parameters $\theta$ for a set of given observations. The goal of MLE is to find the parameters that describe the data set. Since we assume the $x_{i} \cdots$ to be independent RVs, the following can be writen:

$$
f\left(x_{1}, x_{2}, x_{3}, \ldots \mid \theta\right)=f_{1}\left(x_{1} \mid \theta\right) f_{2}\left(x_{2} \mid \theta\right) \cdots f_{n}\left(x_{n} \mid \theta\right)
$$

In other words, it can be written as:

$$
f\left(x_{1}, x_{2}, x_{3}, \ldots \mid \theta\right)=\prod_{i=1}^{n} f\left(x_{i} \mid \theta\right)=\mathscr{L}\left(\theta \mid x_{i}\right)
$$

Equation (3.10) is called the Likelihood Function for an estimation. The goal is to maximize this function in order to obtain the needed parameters. In order to find the maxima or minima of a function, first step is to determine the derivative of the function, which, in this case, is:

$$
\ln \mathscr{L}\left(\theta \mid x_{i}\right)=\sum_{i=1}^{n} f\left(x_{i} \mid \theta\right)
$$

Equation (3.11) is called the Log-likelihood Function for an estimation. This function equals zero at an extrema of the likelihood function. The solution for this equation may not be linear and unique, or even exist. Iterative techniques have to be used in order to come up with an answer.

\section{Advantages}

- Ease of use and interpretation makes MLE a preferred method. 
- Good asymptotic properties allow for a high probability (close to 1) for $\theta_{M L E}$. In other words, as number of data points $n \longrightarrow \infty$ probability becomes close to 1 .

- It has a low variance when converged, in other words, has a low error in estimated parameters.

- It is insensitive to re-parameterization. For example, if $g\left(\theta_{M L E}\right)$ is an estimate for $g(\theta)$ for a function $g$, estimate for $g\left(\theta^{2}\right)$ will be $g\left(\theta_{M L E}^{2}\right)$.

- When converged, it gives the best possible estimate of $\theta_{M L E}$.

\section{Disadvantages}

- Existence and uniqueness are not guaranteed, i.e., the estimated parameter $\theta_{M L E}$ may not be unique or may fail to exist.

- MLE is a point estimate and hence does not represent any uncertainties.

- Sometimes, over-fitting of data may cause problems by assigning zero probability to certain events.

Once the values of $k$ and $c$ are obtained, wind speed probabilities are calculated using eqautions discussed in Section 3.2. Wind speeds at each point in time are considered to be independent $R V s$, meaning that there is no correlation between each subsequent wind speed, or, in other words, wind speed at any given point in time is not affected by speeds in the past observations.

The assumption that wind speeds at each point in time are independent RVs, allows for simplicity in calculating the parameters for Weibull density for each point of interest in time. The wind speeds are assumed to be independent RVs at each point in time for mathematical simplicity that MLE offers. Defining the correlation between each points is a rigorous analysis and is not a goal of the thesis.

\section{Simulation Setup for Wind Speed Prediction}

In order to calculate the probability of different wind speeds, the following steps were taken: 
1. Divide the Historical Wind Speed Data into Desired Time-frames: In this case, the data is divided into four seasons. Each day in season is then divided into 241 hour long time-slots, giving 12 data points for each hour and a total of 288 points per day.

2. Obtain Normalized Frequency Plots of Data: Once the data is divided into desired time-slots, a histogram of data points was made. This was then normalized using the total number of data points.

3. Calculate Probability Using Weibull PDF: Assuming a weibull density, parameters $k$ and $c$ of the density are estimated using Maximum Likelihood Estimation. Predefined functions in MATLAB was used for MLE. Using the parameters obtained, PDF expressions for these parameters was obtained for each 1-hour slot. This curve was then integrated around its peak to calculate the probability of most likely wind speed

\subsubsection{Simulation Results}

Using Maximum Likelihood Estimation (MLE), the parameters $k$ and $c$ parameters best fitted to the data set were obtained. The following simulation results are obtained:

- Before the probability results, PDF curves obtained from different values of $k$ and $c$ parameters are shown.

- Most probable wind speeds and relevant probabilities for all four seasons using Weibull density are also shown. 


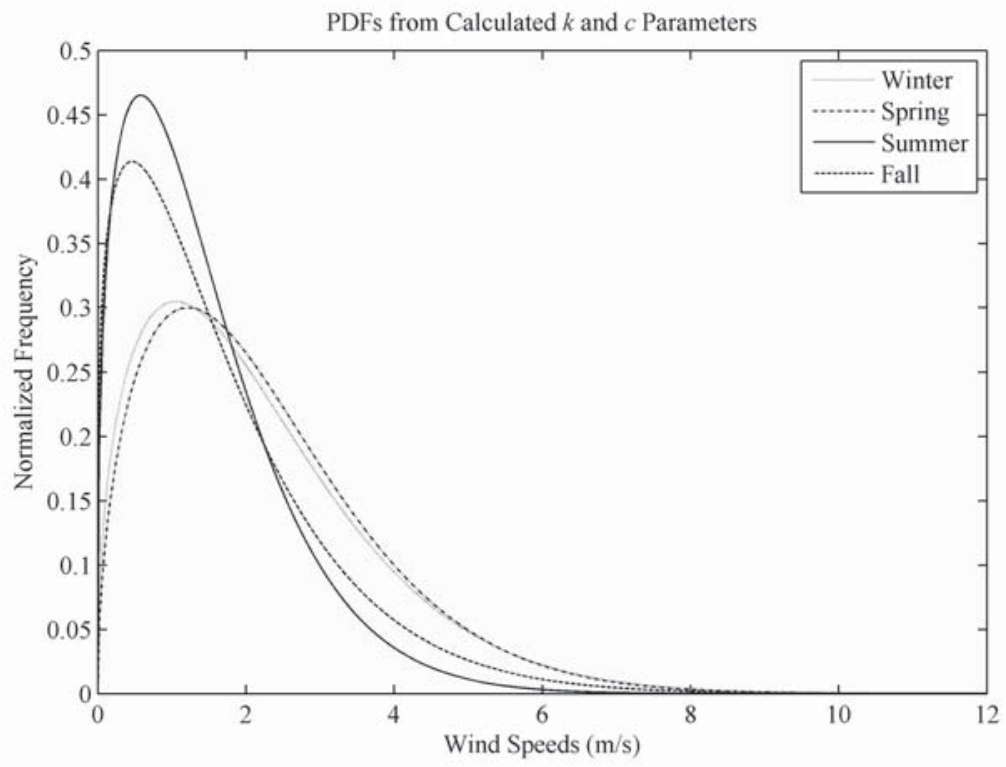

Figure 3.8 - PDF Curves Obtained from $k$ and $c$ Parameters

Figure 3.8 shows Weibull probability curves obtained from MLE for midnight. Data from each hour of the day will generate Weibull density parameters to produce such a plot. Refer to Table A.1 for values of $k$ and $c$ for each hour.

\section{Calculation of Probability Using Weibull PDF}

Once parameters of each time-slot are obtained from MLE, the PDF curve is integrated around its peak for the chosen bin size, giving the most likely wind speeds. 


\section{Most Likely Wind Speeds}

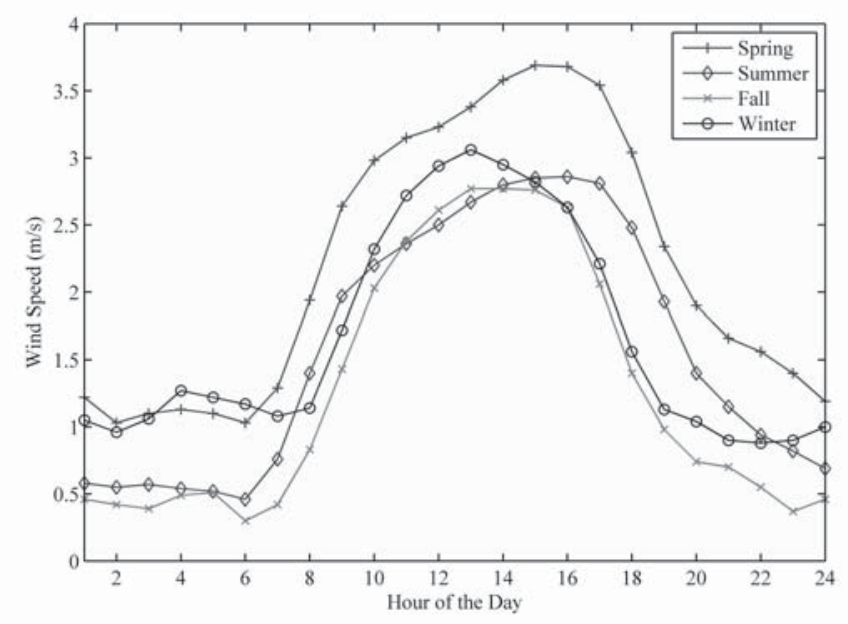

Figure 3.9 - Wind Speeds with Highest Weibull Probability

Figure 3.9 shows wind speeds with highest probability for all 24 hours through out each season. It is interesting to see that although the wind speeds follow a similar pattern, there is a difference in speeds in all four seasons. It is also notable that wind speeds in the geographic location under scrutiny tend to peak during the middle of the day.

Since the area under the PDF curve is 1, integrating the curve over a specific part of the region gives probability of wind speed being between the two limits of the integration (See Equation (3.6)). The more the of the area under the curve is covered, the higher the probability of wind speeds falling in this bracket.

Wind speeds following Weibull densities are known to have only one peak. This feature was utilized in determining the most likely wind speed. However, probability distribution for wind speeds has a broad spectrum, which is the primary reason for even the most likely wind speed to have a low probability.

\subsection{Probabilities Using Nonparametric Statistics}

Nonparametric statistics is the second method used to calculate the probabilities of various wind speeds. This method to calculate probabilities, as opposed to the parametric 
approach, is much simpler. The term nonparametric statistics can refer to the following:

- Distribution-Free Methods: Distribution-free methods assume no underlying assumption about the probability is made. This means that the model in question is entirely data driven.

- Nonparametric Mathematical Models: Nonparametric mathematical models include nonparametric regressions and nonparametric hierarchical processes such as Dirichlet processes.

$$
P_{\text {nonparam }}=\frac{n_{\text {hist }}}{N}
$$

where,

$n_{\text {hist }}=$ Number of data points in a bar of histogram, and

$N=$ Total number of data points

Nonparametric methods have the following advantages:

- simplicity and ease of interpretation for most of the applications, and

- more modifiable due to lack of parameters

Disadvantages include:

- They may be too general for several applications and lack the statistical power that parametric methods have.

- Achieving the same results as parametric statistics can be exhaustive.

Histograms are the simplest form of nonparametric statistics. Equation (3.12) gives the basic idea of a nonparametric method. This method is used to calculate probabilities of each wind speed based upon the total number of occurrences.

\section{Simulation Setup for Wind Speed Prediction}

Various functions were written and MATLAB statistics toolbox was used to implement some of the inbuilt functions. The nonparametric methodology described above is simulated in MATLAB and probabilities of various wind speeds are obtained. This section discusses the simulation results obtained and comments on the factors affecting the simulation setup. In order to calculate the probability of different wind speeds, the following steps were taken: 
1. Divide the Historical Wind Speed Data into Desired Time-frames: Similar to the parametric case. the data is divided into four seasons. Each day in each season is then divided into 24 1-hour long time-slots, giving 12 data points for each hour with a total of 288 points per day.

2. Obtain Normalized Frequency Plots of Data: Once the data is divided into desired time-slots, a histogram of data-points was made. This was then normalized using the total number of data points.

3. Calculate Probability Using Nonparametric PDF: No underlying probability distribution function is assumed. This approach is purely data driven. The probabilities are calculated using the Equation 3.12

\subsubsection{Simulation Results}

As discussed earlier, nonparametric statistics is a data driven way of calculating probability of a certain event. For obtaining the most probable wind speed using this technique, Equation (3.12) is used. In the histogram plot, the bar with highest number of probability has the highest probability of occurrence. This way of calculating probability is simpler, however, the in-depth analysis of parameters may get more complicated as earlier indicated.

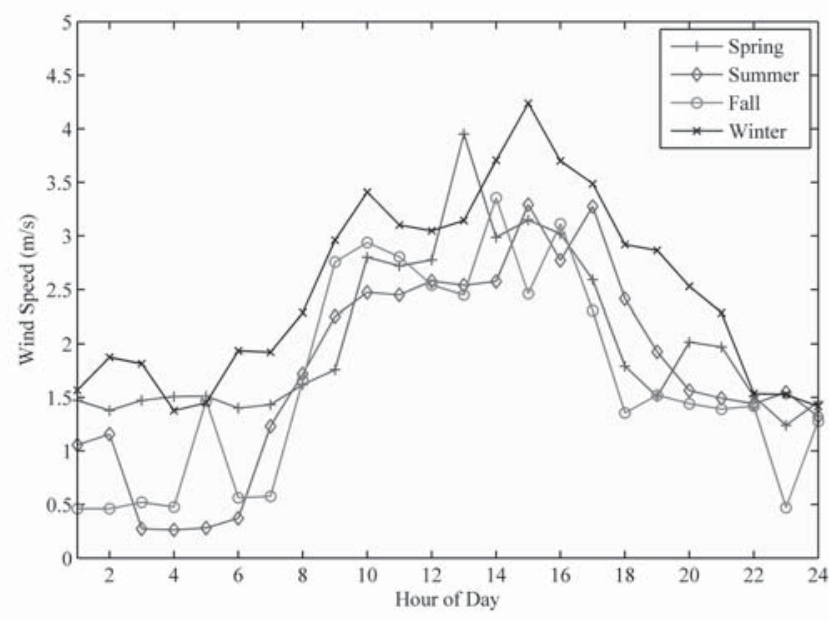

Figure 3.10 - Wind Speeds with Highest Nonparametric Probability 
Figure 3.10 shows the most likely wind speeds calculated from nonparametric statistics. It is easily observed that the change of most likely wind speeds throughout the day is sharper then in figure 3.9. It is also observed that parametric approach produces more conservative results.

\subsection{Comparison of Probabilities Calculated}

Plots discussed above give wind speeds and corresponding probabilities for each of the two methods employed. It is of interest to compare the two methods used. This section compares the results from both methods.

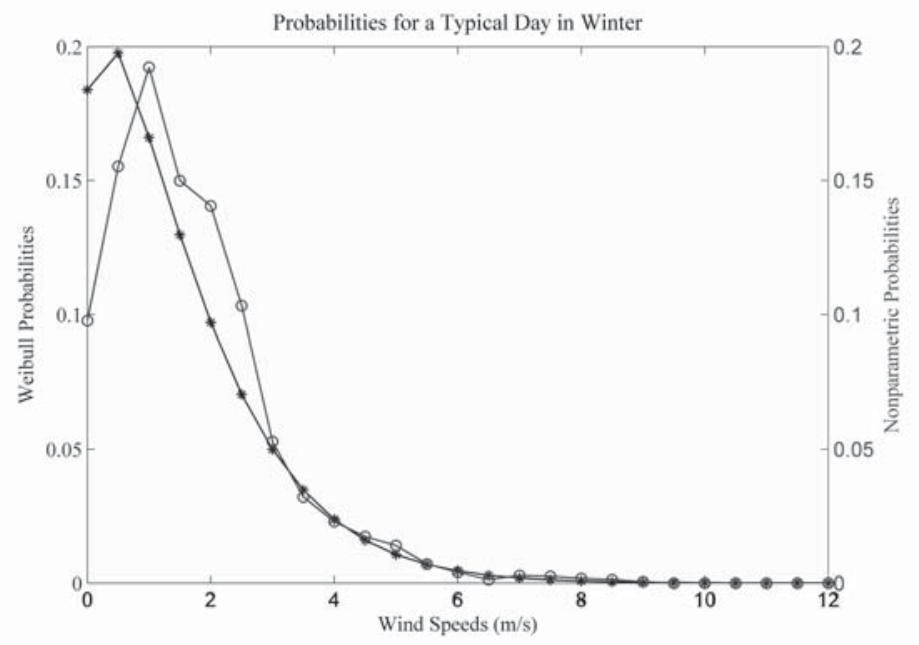

Figure 3.11 - Comparison of Calculated Probabilities for All Wind Speeds

Comparison of probabilities calculated by both the methods used above is made. Plotted above in Figure 3.11 is the comparison for calculated probabilities of all the wind speeds observed in a typical Fall day. The results indicate that both the methods are quite comparable. It should also be noted here that results from parametric statistics are more conservative as compared to the results from nonparametric statistics. Similar results for the other three seasons were also obtained. It was observed that the results for ther three seasons have a similar comparison. A table with all of the probabilities 
for each wind speed on a typical day in each season is given in the appendix (See Table A.2).

\subsection{Summary}

The aim of this section was to cover the fundamental principles used to predict wind speeds from historical data. This chapter covered the following:

1. CDF and PDF functions and their relevant properties were explained.

2. Historical data analysis for probability calculations was made.

3. Calculation of both parametric and nonparametric probabilities for various wind speeds was carried out and results were compared. 


\section{Chapter 4}

\section{Estimating Wind Generation Capacity}

Once the probabilities of various wind speeds are calculated, the next step is to calculate the output of each wind turbine and include the probabilities in the results. Fundamental equations of calculating wind power for a given wind turbine at a particular wind speed have been discussed in Chapter 2. Using the probability calculated above along with the power Equation (2.2), average power output over the next hour for a given turbine is predicted.

The methodology employed to calculate probabilities of individual wind speeds is explained in Chapter 3 along with simulation results. Probabilities obtained using both parametric and nonparametric statistics are utilized to calculate average hourly power prediction.

This chapter describes the method and assumptions used to calculate power output from wind turbines used for the thesis. The probabilities calculated are then used as weights to estimate the power output from wind generation. Figure 4.1 shows the steps taken in order to estimate the power output from wind generation. Power output can be calculated using Equation (2.2) discussed earlier in Chapter 2.

\subsection{Turbine Power Output}

Once the probability is obtained, the next step would be to calculate the power output. Power output of wind turbine is of interest and wind speed is the key component. Figure 


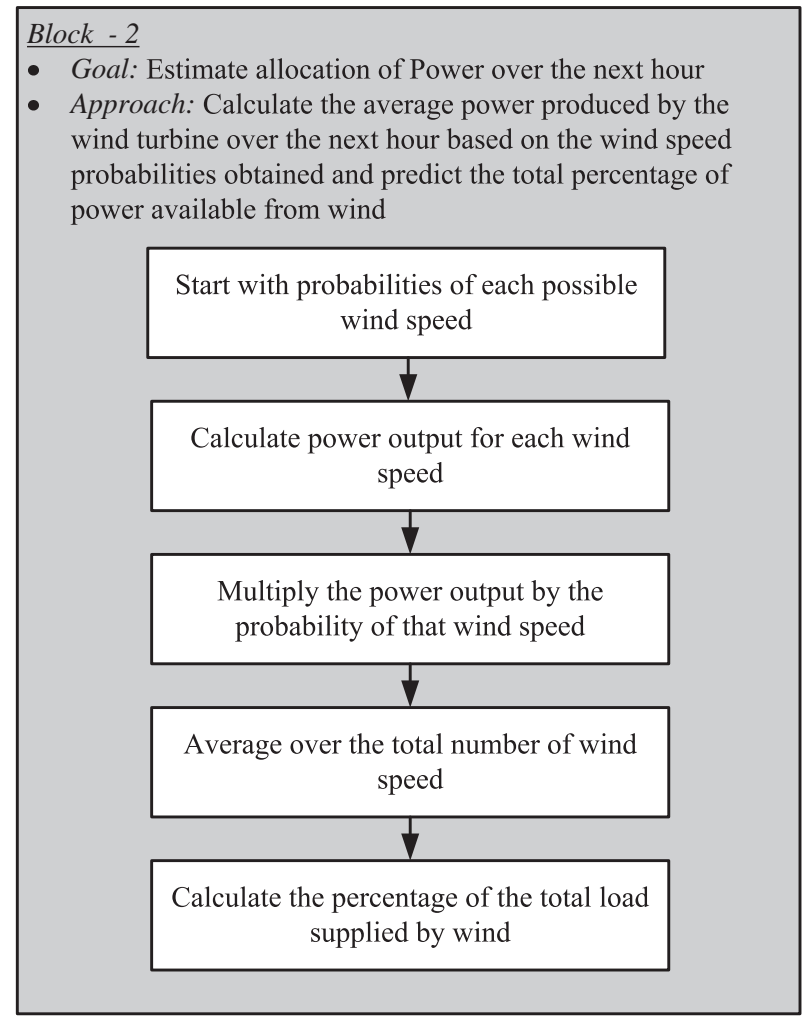

Figure 4.1 - Estimating Wind Power Generation

4.2 shows an output curve of a wind turbine. The curve shown here differs for each turbine depending upon their ratings and makes. Some important points in the curve are:

- Cut-in Speed: $V_{C I}$ is the cut-in wind speed, the speed at which turbine starts producing power. As the speed increases, the output of the turbine increases since it is ideally proportional to the cube of wind speed.

- Rated Speed: $V_{R}$ is the rated wind speed, the speed at which the turbine produces its rated power. Any further increase in speed does not change the power output.

- Cut-off Speed: $V_{C O}$ is the maximum speed at which the turbine can safely function. If the speed goes beyond this point, turbines are normally cut out of operation. 


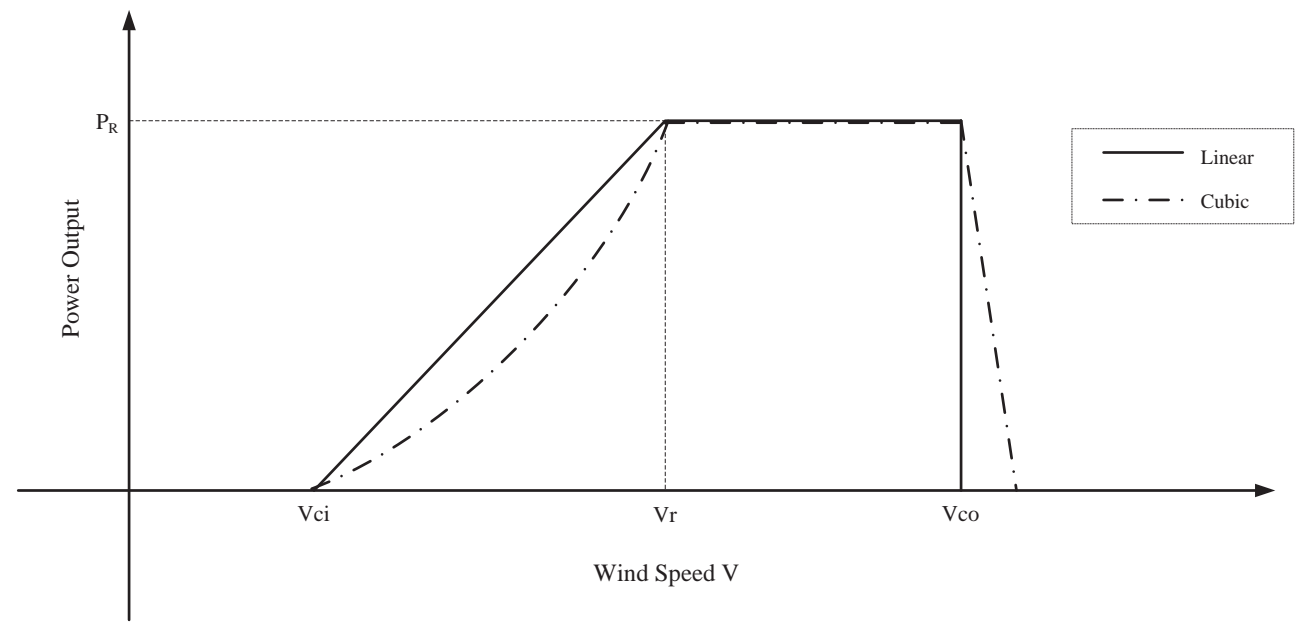

Figure 4.2 - Output of a Wind turbine

Linear approximation can be given as:

$$
P_{W, \text { Linear }}(V)= \begin{cases}0 & \left(0 \leq V_{W} \leq V_{C I}\right) \\ P_{\text {rated }}\left(\frac{V_{W}-V_{C I}}{V_{R}-V_{C I}}\right) & \left(V_{C I} \leq V_{W} \leq V_{R}\right) \\ P_{\text {rated }} & \left(V_{R} \leq V_{W} \leq V_{C O}\right)\end{cases}
$$

Cubic approximation is given by:

$$
P_{W, \text { Cubic }}(V)= \begin{cases}0 & \left(0 \leq V_{W} \leq V_{C I}\right) \\ \frac{1}{2} \rho A V_{W}{ }^{3} C_{P} \eta_{e q} & \left(V_{C I} \leq V_{W} \leq V_{R}\right) \\ P_{\text {rated }} & \left(V_{R} \leq V_{W} \leq V_{C O}\right)\end{cases}
$$

where,

$\eta_{e q}=$ Equivalent efficiency of mechanical and electrical energy conversions combined

The two approaches generally used to approximate power between $V_{C I}$ and $V_{R}$ are linear and cubic (See Figure 4.2). Power output of turbine for each wind speed can be calculated using either of the Equations (4.1) and (4.2). Cubic approximation for the rising power curve between $V_{C I}$ and $V_{C O}$ are frequently made, since the mechanical power produced is proportional to the cube of wind speed. However, in order to simplify, 
a number of researchers [29] use linear approximation. It is also evident from the curve that power output for speeds below $V_{C I}$ and above $V_{C O}$ is zero. Rated power output is only between rated speed $V_{R}$ and cut-off speed $V_{C O}$. Equations (2.2) only gives the mechanical torque produced by the wind turbine, efficiencies of components that follow the turbine, i.e., gear boxes, drive train, rotor and converter all have a cumulative effect on the electrical output of the wind turbine.

\section{Adjustment of Speeds with Hub Heights}

One concern with the available data is low wind speeds. However, it should be noted that the change in wind speeds with varying hub height using Equation (4.3) Assuming that the wind speeds measured are close to ground level, adjustments for a hub height of $65 \mathrm{~m}$, which is close to the average height for turbine ratings used, are made. Using the adjusted speed, average power output for the next hour is predicted. Wind speed was adjusted using:

$$
V_{Z}=V_{i}\left(\frac{Z}{Z_{i}}\right)^{\alpha}
$$

where,

$Z=$ Metering mast height,

$Z_{i}=$ Hub height,

$V_{Z}=$ Velocity at height $Z$,

$V_{i}=$ Velocity at height $Z_{i}$, and

$\alpha=$ Hellman's coefficient.

The Equation (4.3) gives adjusted wind speed to the corresponding hub height. For the historical data used, it is assumed that the speed is a near-ground speed and change in the wind speeds will be accounted for by using Equation (4.3).

\subsection{Average Power Output Prediction}

Average power output over the next hour is of interest here. Calculation of electrical power output and influential factors have been discussed in the previous section. This section aims at calculating predicted average power output of the wind turbine over an 
hour in a particular season.

$$
P_{\text {avg }}=\frac{1}{n} \sum_{i=1}^{n}\left(P_{V_{i}} p_{i}\right)
$$

where,

$P_{\text {avg }}=$ Average power predicted over next hour,

$n=$ total bins,

$P_{V_{i}}=$ Power at wind speed $V$, and

$p_{i}=$ probability of wind speeds in bin $i$.

Equation (4.4) shows how average power output over the next hour can be predicted. Power output at each wind speed occurred is calculated using parameters and equations discussed above. For speeds $0-12 \mathrm{~m} / \mathrm{s}$ ground speed and increment of $0.5 \mathrm{~m} / \mathrm{s}$ for each bin, a total of 25 bins are there. Parametric and nonparametric probabilities calculated in Chapter 3 are used as weights applied to the corresponding power output at a particular wind speeds. The result of this calculation gives average power output to be expected for the next hour in a particular season.

\subsection{Simulation Results}

The goal is to calculate average power based on probabilities calculated in Chapter 3 . For this, first, power output of the turbine parameters used in the distribution system model is simulated. In order to calculate average power output, the following steps were taken:

1. Adjustment of Wind Speed: Wind speed is adjusted for hub height using Equation (4.3)

2. Power Output: Power output is calculated using Equation (2.2)

3. Average Power Calculation: Average power is calculated using Equation (4.4)

\section{Simulated Power Curve}

The adjustments stated above were used for the wind speeds observed in the geographic region under scrutiny. 


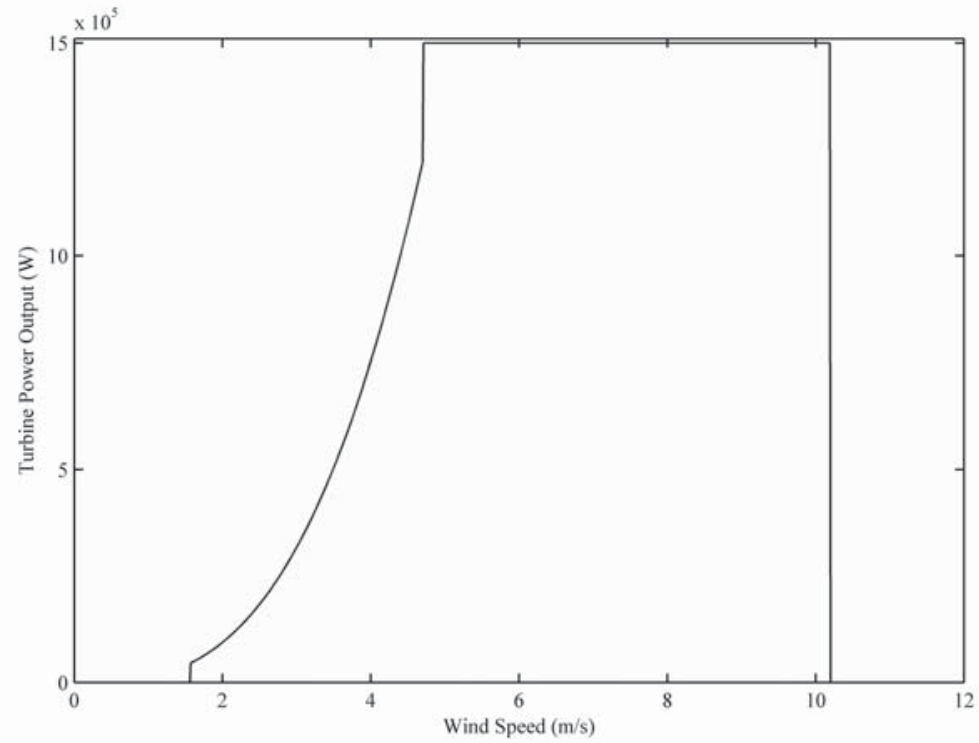

Figure 4.3 - Simulated Output of the Wind turbine

Power output using a cubic approximation (See Equation (4.2)) was simulated for the 1.5MW turbines used in test case developed in Chapter 5. The simulated power curve is shown in Figure 4.3. The height of the measurement mast is taken to be $10 \mathrm{~m}$ [52]. A hub height of $65 \mathrm{~m}$ is assumed for adjustment of wind speed in accordance with height. The parameters used for calculation of power output of a $1.5 \mathrm{MW}$ turbine are given in Table A.3. A similar output curve is obtained for a $1.5 \mathrm{MW}$ turbine using the in-built DFIG model in MATLAB. 

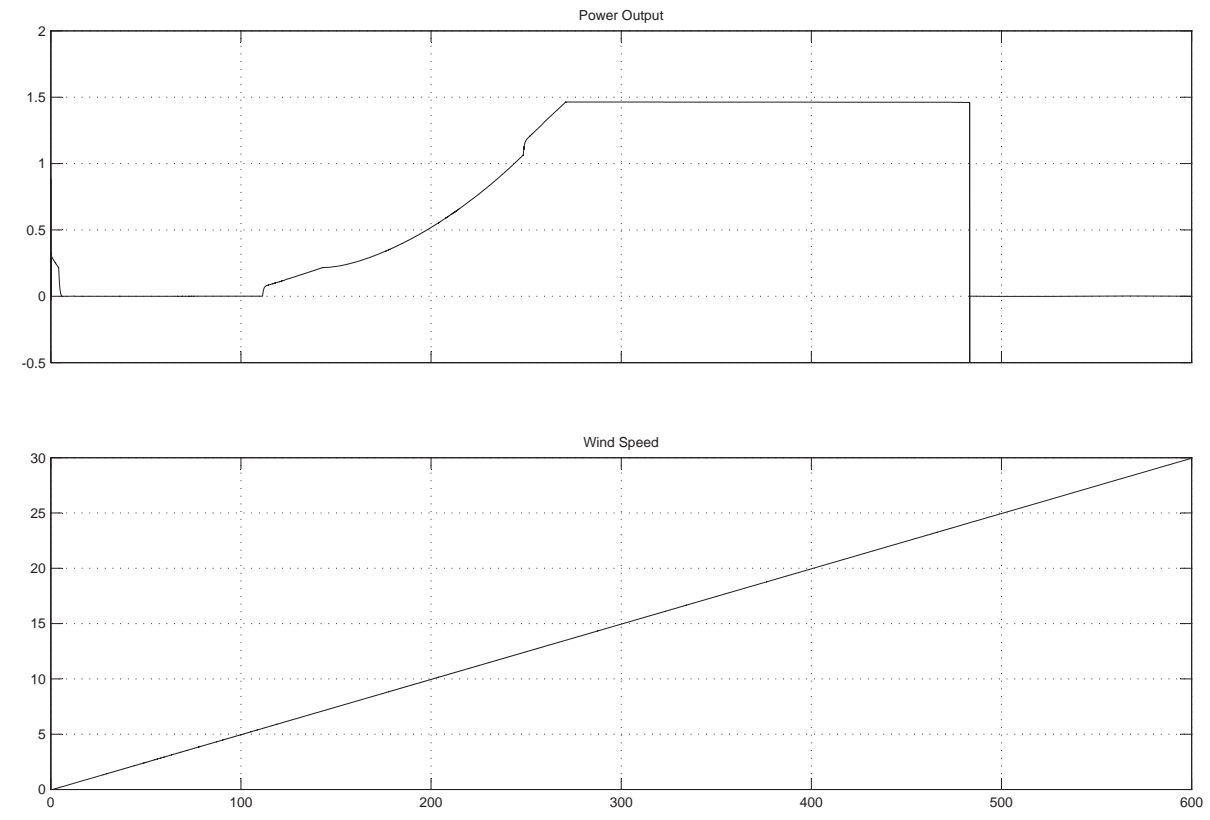

Time offset: 0

Figure 4.4 - Output of a Wind turbine from In-built MATLAB DFIG Block

Figure 4.4 shows the output of the in-built wind turbine model in MATLAB Simpowersystems. Wind speed is slowly increased from 0 to $V_{C O}$ of $30 \mathrm{~m} / \mathrm{s}$ at the rate of $0.01 \mathrm{~m} / \mathrm{s}$ over a period of 10 minutes. It can be observed that the turbine output has a small transient in the beginning, which is due to in-built simulation parameters. The output remains zero until the cut-in speed $V_{C I}$ is exceeded. It should be noted that the output never reaches full $1.5 \mathrm{MW}$ and stays at a slightly lower point. This is not the case in practice, as full nameplate capacity can be achieved for ambient conditions. It should also be noted that the transition of output from below rated to rated is not smooth.

\section{Predicted Average Power Outputs}

Based on the probability calculations of Chapter 3 (See Figures 3.9 and 3.10), it is found that the probability distribution of wind speeds is very wide. Even the maximum proba- 


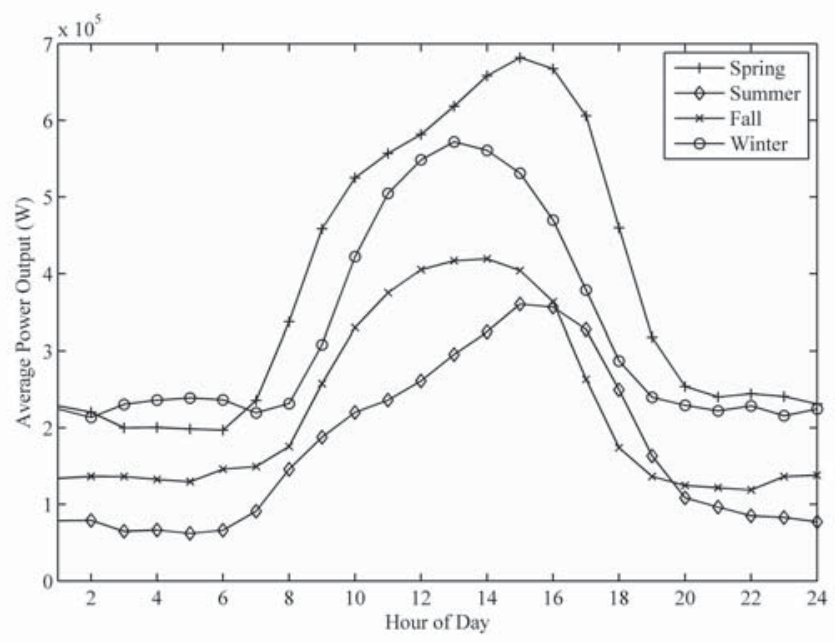

Figure 4.5 - Predicted Average Hourly Output of the Wind Turbine - Weibull Probability

bilities on the curve range between 0.14 to 0.2 (See Figure 3.11). This goes on to show that there is no dominant probability. In order to incorporate these probabilities into the power output of wind generation at different wind speeds, probabilities of each wind speed are used as weights to relevant power output at that speed. Taking an weighted average of the output gives a more useful perspective for long-term planning, especially, when analyzing the potential of a particular site for wind generation.

Using Equation (4.4), average hourly output for each season is predicted. Figure 4.5 shows the results of anticipated average power outputs from wind turbines in the given geographic location.

Figures 4.5 and 4.6 show the calculated power outputs for $1.5 \mathrm{MW}$ turbine model with parameters discussed in Table A.3.

The probabilities used here are Weibull probabilities calculated earlier in Chapter 3. It is easily seen that the maximum average output is about $0.65 \mathrm{MW}$. This is because, for the given geographic location, higher wind speeds have much lower probabilities. Since probabilities are the weights used here, the average output is still small.

Figure 4.6 shows a similar results for nonparametric probability, also calculated earlier in Chapter 3. An interesting observation about the results is that the results obtained from parametric probability calculation are more conservative compared to the results from nonparametric probability. In other words, nonparametric probability predicts a 


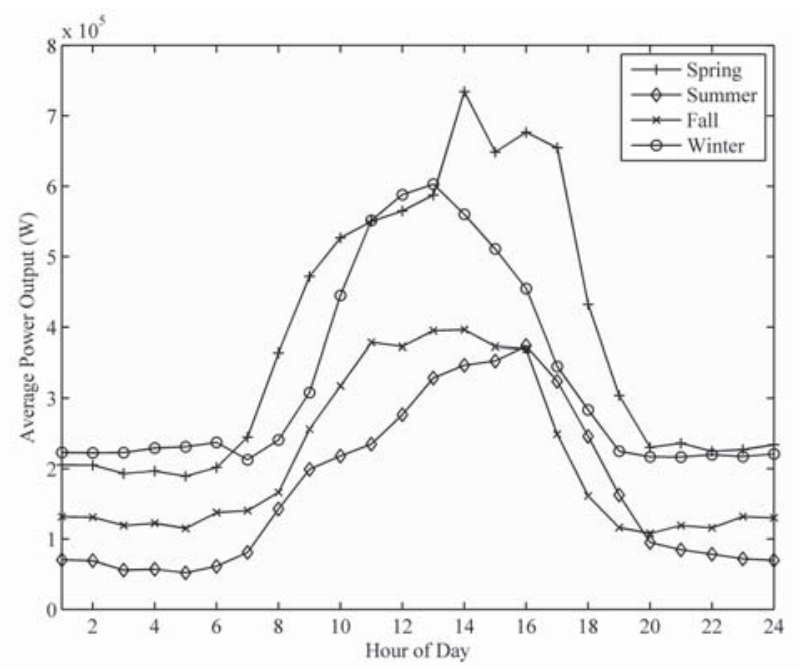

Figure 4.6 - Predicted Average Hourly Output of the Wind Turbine - Nonparametric Probability

much higher average power output for the same speeds in comparison to parametric probability.

\subsection{Summary}

In this chapter, electrical output of the $1.5 \mathrm{MW}$ turbines used in simulations later was obtained. Probabilities were, then, applied as weights to this calculated power outputs to predict power output for the next hour. Two sets of probabilities, calculated using parametric and nonparametric methods, were then used to calculate predicted average power output for any hour of the day. To conclude, the following was accomplished in this chapter:

1. Power output of $1.5 \mathrm{MW}$ turbines that are be used in test system in Chapter 5 was calculated.

2. Calculated probabilities (Chapter 3) into calculation of anticipated power output for each hour in each season were incorporated in average power output calculation. 


\section{Chapter 5}

\section{Enumeration of Switching Combinations}

The goal of this chapter is to enumerate feasible switching combinations and incorporate the wind speed probabilities calculated in Chapter 3. Identification of a suitable switching combination for different operating scenarios is desired. A test distribution system with 29 nodes, two wind generation facilities and 8 remote controllable switches is designed. All possible switching topologies are assessed in order to reconfigure the network under different operating conditions. Basic feasibility constraints are defined to identify feasible network topologies and reject those that are unfeasible in advance, with an aim of reducing computational effort. Scenarios where wind generation directly affects the test system are also analyzed. 


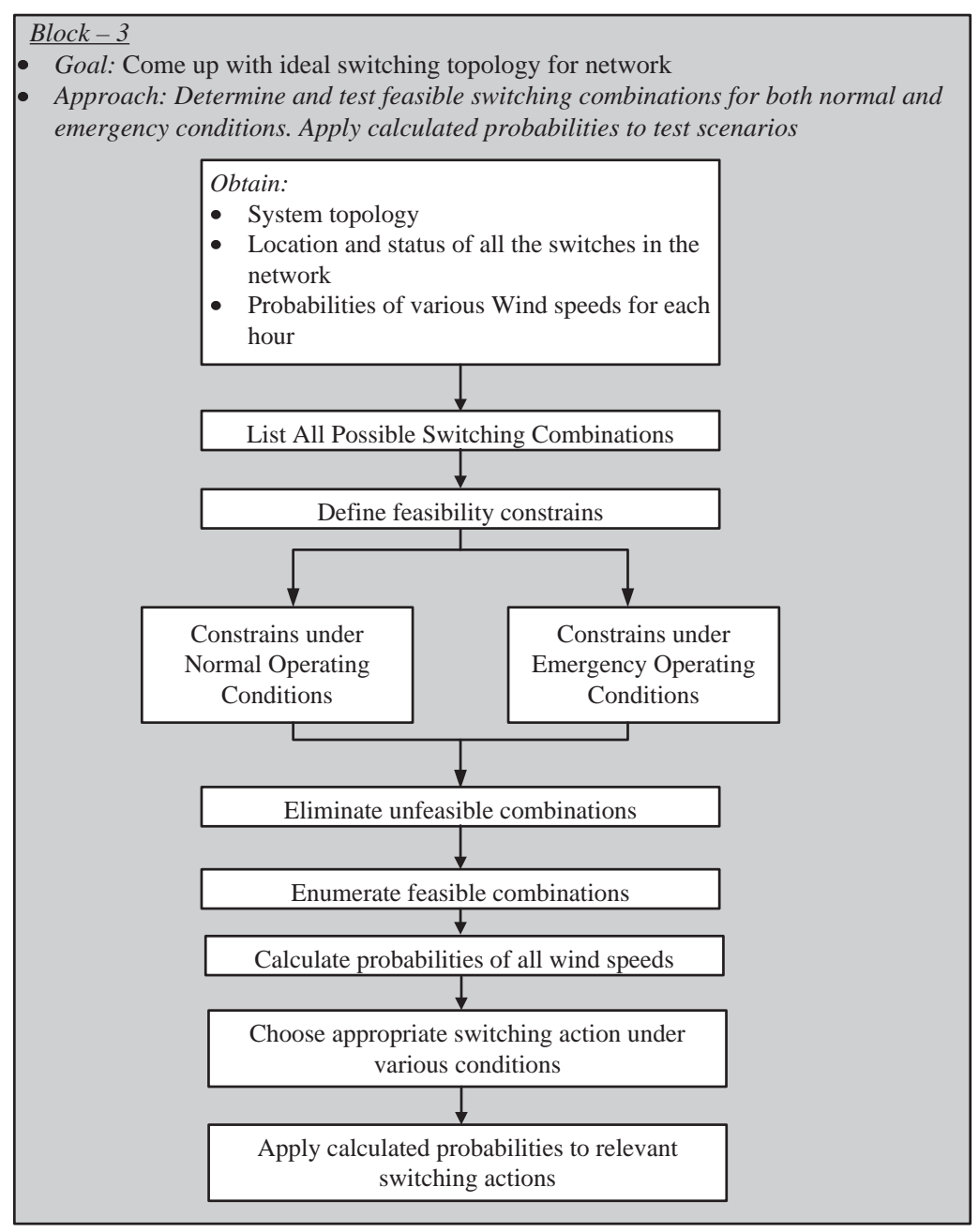

Figure 5.1 - Enumeration of Switching Combinations

Probabilities calculated in Chapter 3 are applied to the wind generation in the system. The probabilities are then linked with capacity provided by wind generation under various situations where the system experiences loss of generation on the grid side. 


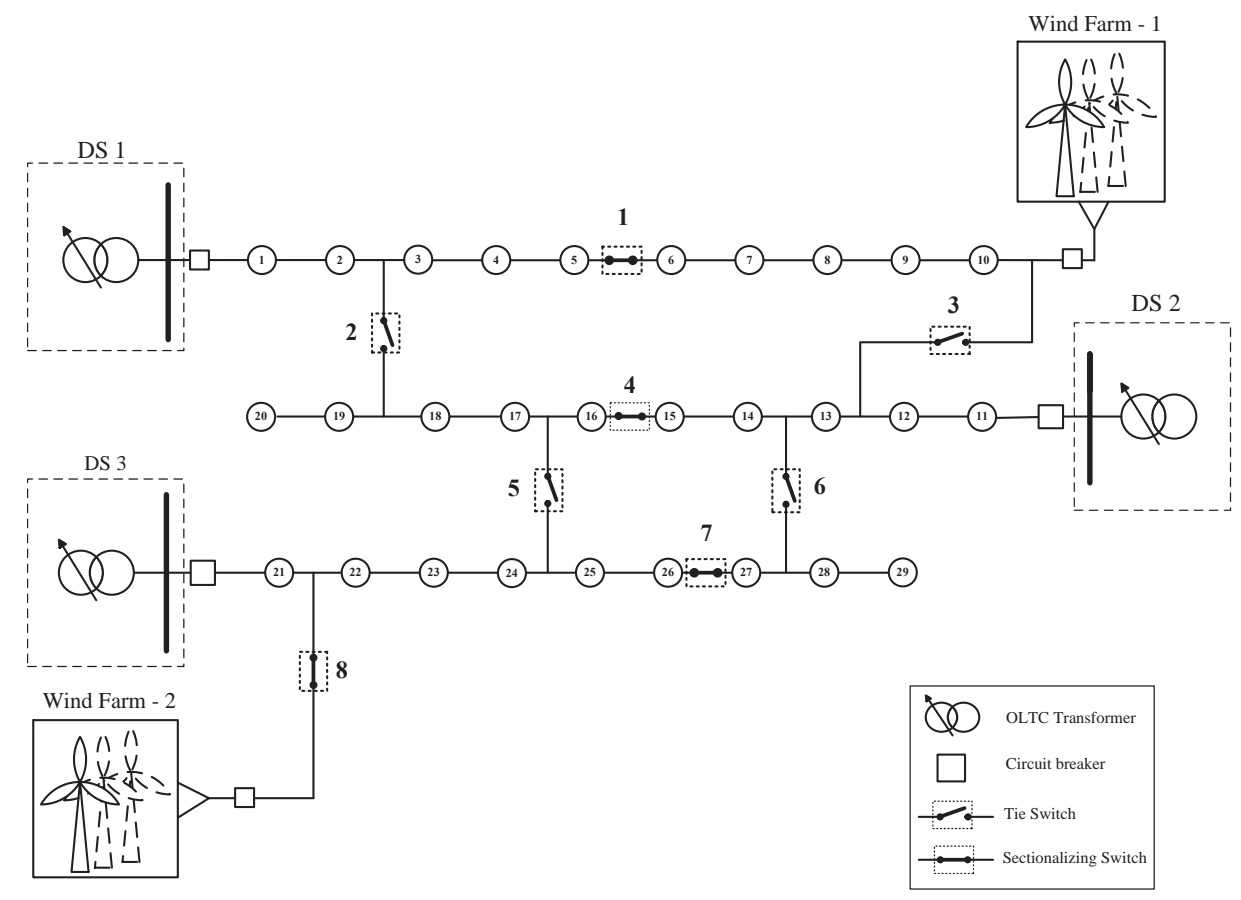

Figure 5.2 - Base Case with Wind DG

\subsection{Test System Description}

In order to better illustrate the definition of feasible switching combinations and inclusion of probabilities in various conditions, the system topology used for this thesis is discussed here. Figure 5.2 shows the topology of the test system. The basic system consists of 29 nodes and three-feeder. Two wind farms, one of 18MW and he other of $9 \mathrm{MW}$, are also present in the system. Together their total capacity adds up to $40 \%$ of total system load. Provision of alternative paths for serving loads is made by 8 remote controlled switches in the system. In the default topology shown in the system, 4 switches are tie switches and 4 switches are sectionalizing switches (See Section 2.5.1 for definition).

In the subsequent sections, total possible switching combinations will be discussed followed by definition and application of feasibility constraints under both normal and emergency conditions. The chapter will conclude with simulation results. 


\subsection{Switching Combinations}

Basic concepts of distribution system reconfiguration have already been discussed in Chapter 2. For a given system topology, the goal here is to list all possible switching combinations and identify feasible ones. First, it is useful to state how all of the combinations are defined for a given topology.

\subsubsection{Possible Switching Combinations}

Let a network consist of a total of $n$ tie and sectionalizing switches. Each of these switches can be assigned two states: 0 for a tie switch (Normally Open switch) and 1 for a sectionalizing switch (Normally Closed switch). Assuming all of the switches in the network are capable of taking either state 0 or state 1 , a total of $2^{n}$ possible combinations (accounting for repetition and order of 0 's and 1 's) exist. Let this number be $C$, then:

$$
C=2^{n}
$$

It should be noted that the number of combinations $C=2^{n}$ includes repetitions and order is important, since switching topologies are of interest. However, for the sake of simplicity, if combinations without taking order into account are considered, the number is much smaller and becomes $n+(2-1)$. It is also evident that not all of these topologies are actually feasible. Feasibility of a combination is defined by fulfilling the basic system constraint discussed in the next section. Mathematically,

$$
C_{\text {feas }}=C-C_{\text {nonfeas }}
$$

where,

$C_{\text {feas }}=$ Feasible switching combinations, and

$C_{\text {nonfeas }}=$ Non-feasible switching combination. 


\subsubsection{Feasible Switching Combinations}

It is desired to identify feasible switching combinations out of all possible combinations. For example, if the objective was to have minimum losses, system losses would be zero if all of the generators are disconnected by switching action. However, this is not a feasible solution, as no load will be served. As a result, a number of constraints must be applied in order for a switching combination to be feasible. The following section discuses the constraints.

\section{Constraints Under Normal Operating Conditions}

Normal operating condition is taken here as a healthy and fault-free state of the system.

1. All load must be served. This must be the main constraint under normal operating conditions. Under emergency conditions, however, unserved load must be at its minimum.

2. Voltages must be within allowable limit at the load.

$$
V_{\text {min }} \leq V_{\text {load }} \leq V_{\text {max }}
$$

The voltage at consumption point must be between its minimum and maximum allowable limits. In distribution systems, the allowable voltage should be between $95 \%$ and $105 \%$ of nominal voltage.

3. Network must be maintained radial. Typically distribution feeders have radial topology. Another reason is that the existing protection coordination is mostly designed for radial networks.

4. Wind generation must be connected to at least one of the substations at all times. A DFIG model is used, which needs grid voltage support and is incapable of stand-alone operation.

\section{Constraints Under Emergency Operating Conditions}

1. Fault must be located and isolated as soon as it is detected. Once the fault has been isolated, reducing the number of customers can be a secondary goal. Isolating the 
fault to a point where least number of customers are affected, can be obtained by proper operation of the remote controllable switches.

2. In an attempt to reduce the number of affected customers, the limits of the components such as feeder capacity, thermal limits of lines and distribution transformers in the system must be considered. Overloading any of them for an extended period of time, while the system recovers from a fault, can lead to catastrophic breakdown of the entire system.

3. If the fault is in a circuit associated with wind generation, wind generation must be immediately disconnected from the system. On top of the fact that the model used here for simulations needs grid voltage support, the wind farms connected to the system under fault conditions may feed the fault making it severe. For these reasons, in the event of a fault on the connected systems, wind farms must be removed from operation.

4. Constraints of a radial topology and voltage limit 5.2.3 still exist for the healthy part of the system.

Under emergency conditions, it is normal utility practice to disconnect the wind turbines. This does not necessarily mean that they cannot operate on stand alone. Also, the constraint of a continuous grid support to wind turbine applied is restricted to the inbuilt MATLAB model used. Once the constraints listed above are satisfied, further constraints can be applied for optimal systems operation. For example, under normal conditions, more than one feasible combinations may exist. A further constraint of minimum loss can be applied to choose the best option out of the available combinations.

\subsubsection{Applying the Constraints}

For the test system under scrutiny, the number of total possible combinations is $C=2^{8}$, giving 256 possible switching combinations. Not accounting for repetition or order, this number is $8+(2-1)=9$. It must be noted that the purpose of mentioning these 9 combinations is for computational ease. The order of combinations here is extremely important since the network topology is of interest. 
To computationally apply the constraints discussed in Section 5.2.2, topology detection algorithms such as those in graph theory can be employed. For this small network, however, no such algorithms were applied and combinations were tested with brute force. By simple visual inspection of the test system (See Figure 5.2), it can be noted that:

- Combinations with all zeroes and all ones can be easily eliminated: all zeroes for not serving all of the load and all ones for encountering the loops.

- Similarly, the sets of combinations which have seven 0's and a 1, or seven 1's and a 0 can also be removed for the same reason, eliminating $2+2 \times\left(\frac{8 !}{1 !}\right)=18$ combinations.

- Another reduction, for the same reason as above, comes from the combinations that have two 1's and six 0's and vice-versa. This is a large reduction that accounts for a total of $2 \times\left(\frac{8 !}{2 !}\right)=144$

- Further eliminations were be made in similar way resulting in a total of 5 combinations with wind generation and 3 more without one or both wind farms- 1 and 2 . However, we are not interested in the combinations that do not have wind farms incorporated and hence the 3 combinations without wind can be disregarded. So, the resultant number of possible combinations is $5+1$ Default $=6$ combinations in total.

- Thus, out of the remaining 94 combination from a total of 256 combinations for the test system, only 6 work under normal conditions.

All 6 of the feasible combinations thus derived are listed in Figure 5.3. The arrows indicate the direction of currents. Switches that are highlighted are closed and those that aren't are open. It should be noted that the combination with no wind generation is still a feasible combination. However, the combinations that include wind turbines not generating are of lesser interest given the theme of this work. 

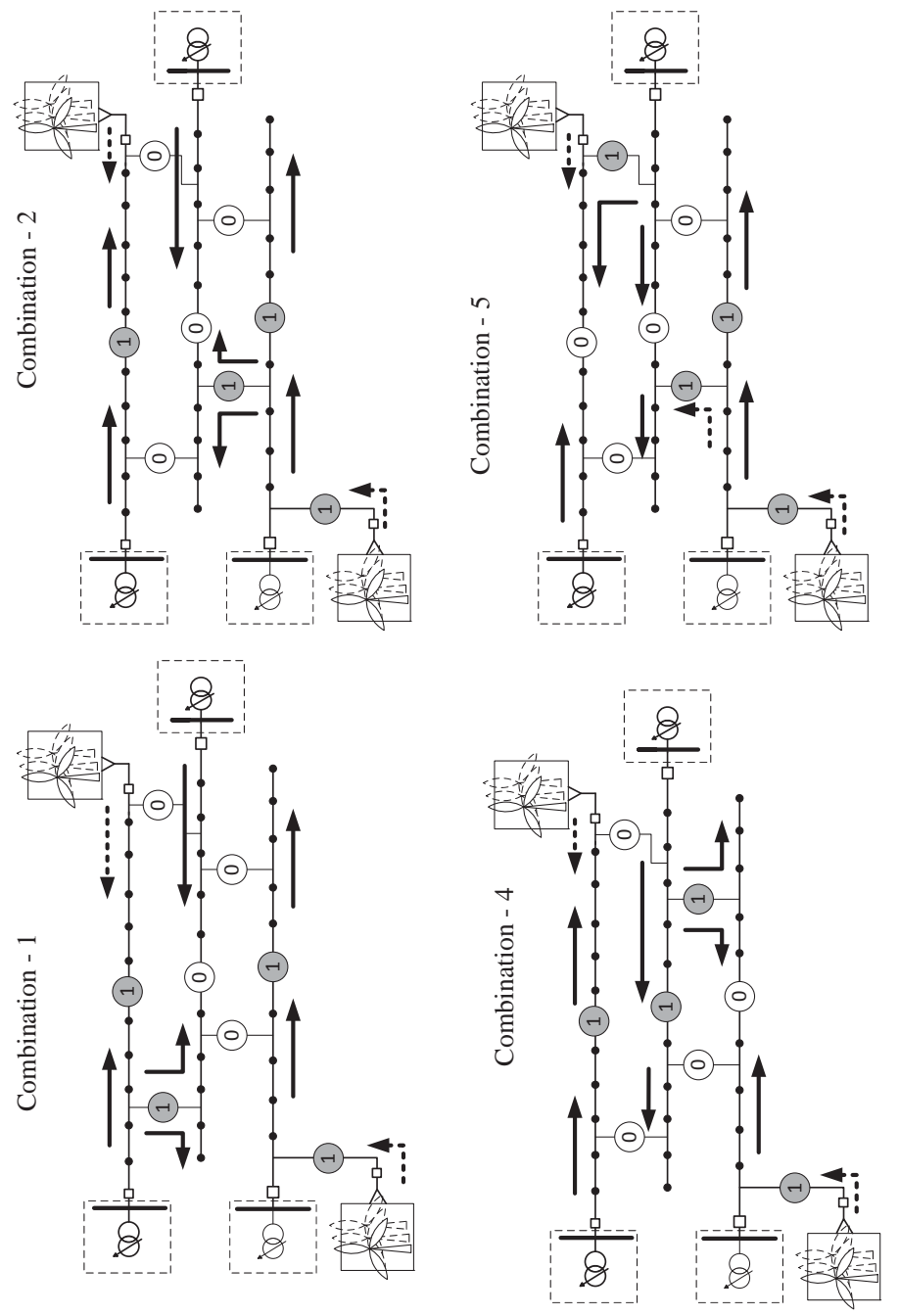

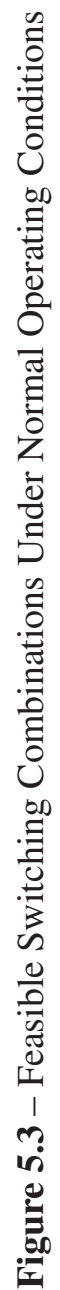
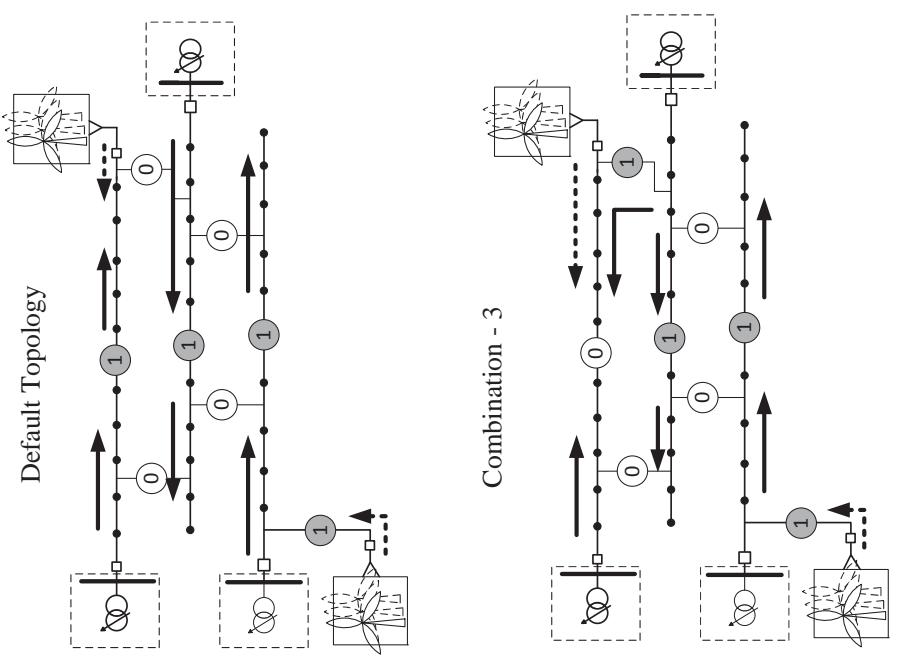


\section{Feasible Switching Combinations Under Normal Conditions}
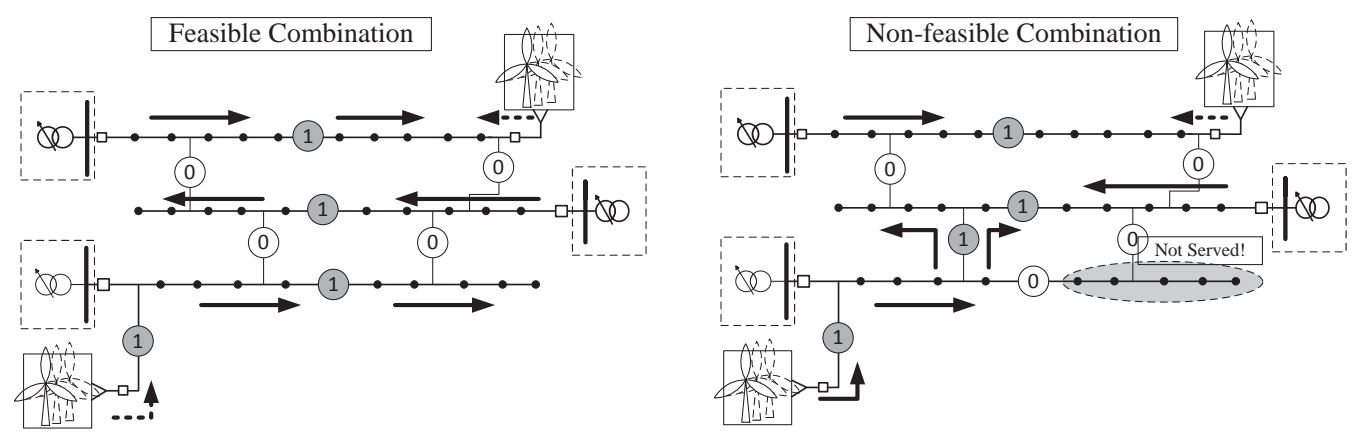

Figure 5.4 - Feasible vs. Non-feasible Switching Combination - Normal Conditions

Figure 5.4 shows a feasible and a non-feasible switching combination under normal operating condition. The feasible combination on the left satisfies all of the constraints listed in section 5.2.2: All of the load is served within the allowable voltage limits and network remains radial. The one on the right, however, is not a feasible combination since:

1. Customers on the circled area are unnecessarily not served.

2. The middle section of feeder 2 is fed by both feeder 2 and feeder 3 , causing a two-way current and potential malfunction.

As indicated earlier, all of the possible combinations were tried for this small system using a brute-force method. This may be highly impractical for larger systems and may require more sophisticated computational algorithms. Potential implementation of the algorithms can be a combination of loop-detection techniques and sub-dividing a large network into smaller parts.

\section{Feasible Switching Combinations Under Emergency Conditions}

Definition of an emergency condition here is taken as a permanent fault at any of the system components. Common types of faults are three-phase to ground, single phase to ground, phase to phase, two phase to ground and so on. 

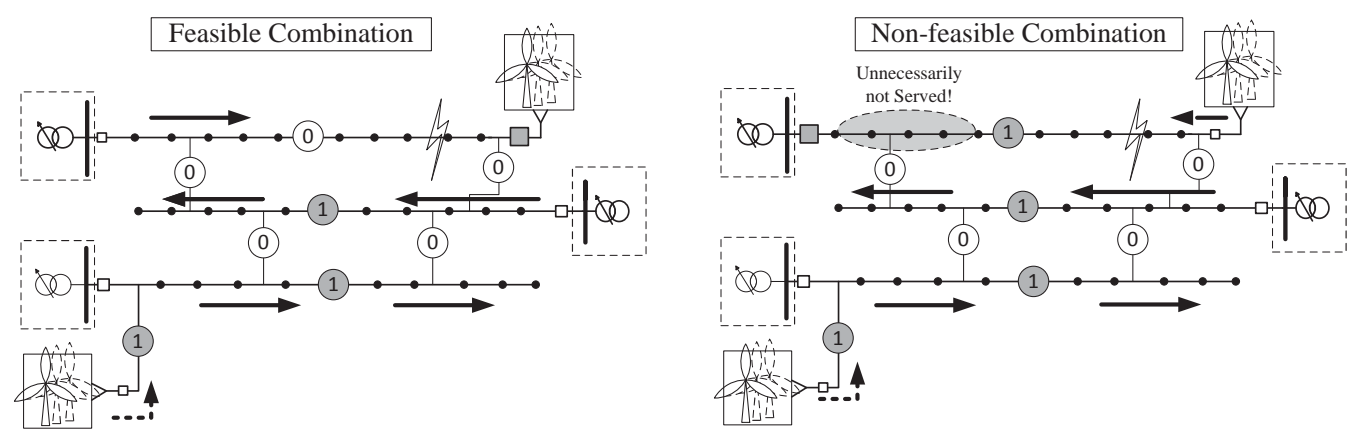

Figure 5.5 - Feasible vs. Non-feasible Switching Combination - Emergency Conditions

Figure 5.5 shows a fault near wind farm-1 and an example of a feasible and a nonfeasible switching combination. The combination on the left is feasible because all of the constraints listed in section are satisfied along with minimum number of customers being affected. The combination on the right is not feasible because:

1. Wind farm has not been disconnected and may possibly be feeding into the fault.

2. Customers on the circled area are unnecessarily not served.

Under emergency conditions in the test system here, the following should be noted:

- Considering each of the node, the associated line section, three feeder heads and two wind farms, disregarding the remote controllable switches, the total number of system components is 34 .

- Consider four types of faults listed above at each of these 34 components for four types of faults, one fault at a time. The number of possible faults is $34 \times 4=132$.

- Further, for example, if two simultaneous faults at two locations were to be considered, total possible number of such combinations is $34 \times 33=1122$.

- Including the 8 remote controllable switches with two states each, even for the 6 feasible combinations discussed earlier, the total number of possibilities would be $(34 \times 4)^{6}$ possible combinations.

This discussion gives the perspective of the depth of analysis required for emergency operating conditions. Hence, it must be emphasised that enumerating all of the feasible 
scenarios under all types of faults requires further detailed analysis and is considered beyond the scope of this thesis. However, in events of faults that cause loss of generation from the grid side, availability of wind generation becomes useful. Three such emergency conditions are considered. Feasibile switching topologies for each of the situation will be discussed in this section. The following is the discussion of switching topologies for the emergency scenarios considered for this thesis:

1. Generation at feeder-1 experiences outage: Figure 5.6 shows two possible switching combinations in case of a complete loss of generation at DS-1. In the combination on the left side, it has been assumed that the wind farm is producing at its rated capacity. The grid support(See Figure A.7)bis provided by operating tie switch 3 (See Figure 5.2). Since the wind farm is producing at its rated capacity, it can take on all of the load for DS-1 for the test system A.7.

Outage at DS-1: Rated Output From Wind

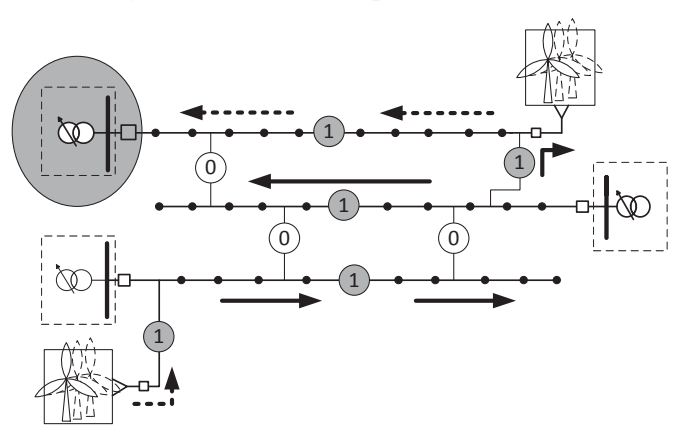

Outage at DS-1: Lower or No Output from Wind

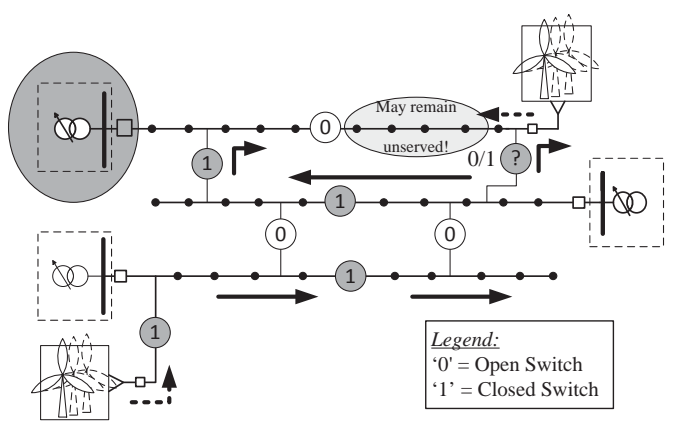

Figure 5.6 - Feasible Switching Combinations In case of an Outage at Feeder DS-1

The combination on the right side shows a situation where the wind farm is producing less then 50\%. Depending upon the status of the switch 3, the circled area may or may not be served. Even so, by opening the sectionalizing switch 1, some of the load on the feeder can be provided. In this case, however, some of the load on the left side of switch 1 can go unserved.

2. Generation at feeder-2 experiences outage: Figure 5.7 shows two possible switching combinations in case of a complete loss of generation at DS-2. There 

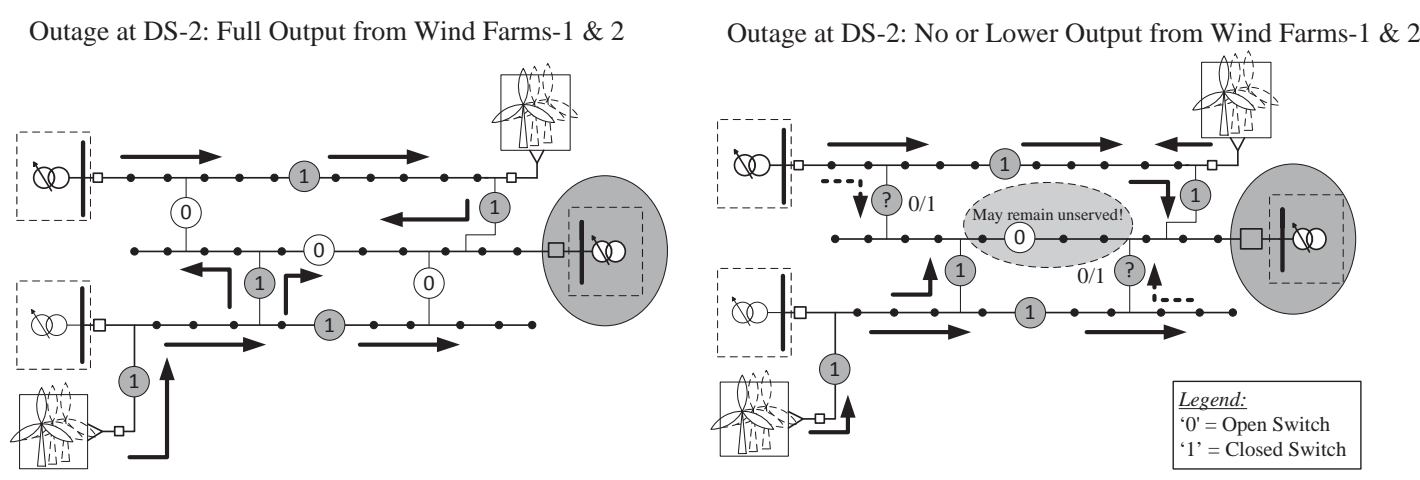

Figure 5.7 - Feasible Switching Combinations In case of an Outage at Feeder DS-2

are no wind farms directly connected to DS-2, but it can still be indirectly connected to both the wind farms by operating tie switches 3 and 5 (See Figure 5.2). In this case, in order to avoid any loops, sectionalizing switch 4 must be operated as well. In an event of full output from wind farms, as shown in combination on the left side, half of the load on the right of switch 4 can be easily supplied by Wind farm-1. Other half can be supplied by wind farm-2 and some capacity from DS-3.

The combination on the right shows a situation when there is little or no output from both the turbines. Here also, depending upon the status of the switches 2 and 6 , the circled area may or may not be served. In this case, the switching combinations are maintained the same, however, since DS-2 has the highest amount of load connected to it (See Table A.7), in an event of no or little output from wind farms, loads on the middle of the feeder (Loads on nodes 13 to 18) may not be served.

3. Generation at feeder-3 experiences outage: Figure 5.8 shows two possible switching combinations in case of a complete loss of generation at DS-3. Wind farm-2 gets direct grid support from Feeder DS-3. In an event of loss of generation on DS-3, wind farm-2 is at a risk of losing grid support and being disconnected. However, this is prevented by operating tie switches 3 (See Figure 5.2) and 6. However in order to avoid a loop with DS-1, the sectionalizing switch 1 also must be operated, as shown in the combination on the left side. 

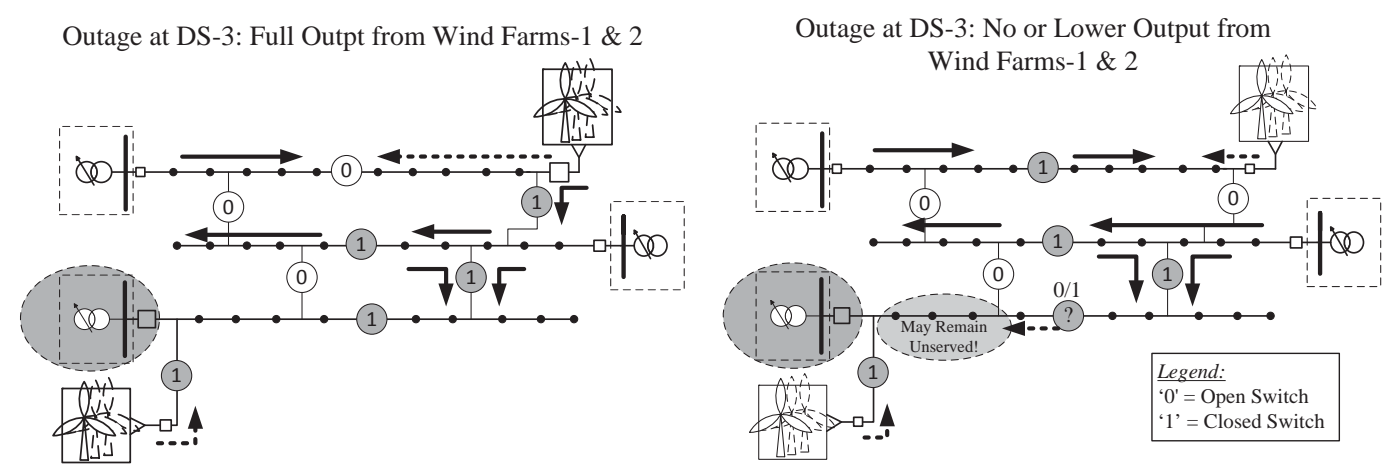

Figure 5.8 - Feasible Switching Combinations In case of an Outage at Feeder DS-3

In an event of a lower or no output from the wind farms, as shown on the right side, load can be served by changing the status of switch 3 (opening the tie switch 3 that was closed earlier). Similar to the two cases above, the status of switch 7 will decide whether the load in the circled area is served or not. However, since DS-3 still has a large amount of load connected to it some of the load may be unserved in either case.

After enumerating the feasible switching combinations for all of the switching combinations discussed above (for both normal and emergency operations), probability of power output from wind farms will be assessed using the technique developed in Chapter 3. Combinations listed here are simulated in the test model. The detailed results are discussed in a latter section.

\subsection{System Setup}

The constraints above along with the predicted power output are applied to a small test system with 29 load-points and 8 switches (including both tie and sectionalizing). The following assumptions were made for the test system in base case:

- The primary network is a $25 \mathrm{kV}$ three-phase network with three feeders. There are three feeder-heads that act as generating buses for the load flow. 
- The distribution substation transformers operate at $75 \%$ of their nominal capacity, in other words, they have $25 \%$ excess capacity in case of an overload. This is generally an adequate capacity as it also avoids heating of the transformers under full-load conditions and thus helps avoid premature equipment failure.

- The work focuses on an hourly time frame and so, for the duration under consideration, loads are considered constant.

Details on the simulation setup and each component and the parameters used are discussed in Appendix A. A very important part of the simulation in MATLAB is the monitoring and protection blocks for both the wind farms, which are discussed in Appendix A.8.

\subsubsection{Required Data}

In order to determine various switching combinations, it is assumed that the following are known:

1. Current distribution system topology

2. Total number of switches

3. Location and status of the switches

4. Existing system capacity

5. Predicted average output of wind turbines

6. Fault location under fault conditions

The monitoring system simulated for each wind farms monitors the following 7 key parameters in the order that they appear in the simulation results:

1. Positive sequence voltage

2. Positive sequence current

3. Average real power, $P$

4. Average reactive power, $Q$

5. Rotor speed 
6. Pitch angle of the blades

7. Wind speed

\subsection{Simulation Results}

As the Figure 5.2 shows, there are a total of eight tie switches in this network: four normally open or tie, and four normally closed or sectionalizing. The topology shown in Figure 5.2 is the default topology of the network.

The first set of simulations displays the outputs from the test system simulated in MATLAB under normal conditions. Later, the concepts used in Chapter 3 are used to calculate the following probabilities:

1. Probability that the wind turbines will produce below rated output

2. Probability that the wind turbines will produce at rated output

3. Probability that the wind turbines will not produce any output

Next section will focus on emergency conditions that affect the grid side generation. It is of interest to calculate the probabilities of wind being able to supply some or all of the load in case of a loss of generation from the grid side. The scenarios considered and suitable topologies are discussed in Section 5.2.3. After simulating the scenarios in the MATLAB test system A.4, probability of support from wind in each of the scenario is calculated. The following probabilities are then calculated:

1. Probability that wind generation will provide no output

2. Probability that wind generation will provide partial output

3. Probability that wind generation will provide rated output

It must be kept in mind that the wind speed data used is for a specific geographical location (Elizabeth City, NC) and hence should not be considered as a generalized result. The probabilities calculated will depend upon the data set for the location under scrutiny. 


\subsubsection{Normal Operating Conditions}

Under normal conditions, serving all of the load is the most important requirement. The rest of this section shows the results obtained from the test system (See Figure 5.2) recreated in MATLAB (See Section A.5). The following is the list of important steps:

- The wind farms in the test system are fed with wind speeds between cut-in $\left(V_{C I}\right)$ and rated wind speed $\left(V_{R}\right)$, rated wind speed $\left(V_{R}\right)$ and between rated wind speed $\left(V_{R}\right)$ and cut-off wind speed $\left(V_{C O}\right)$.

- The voltage of the turbine model in MATLAB is kept constant 1pu for this case.

- Both the wind farms-1 and 2 have grid support from feeders DS-1 and DS-3, respectively.

- The results shown here are only for wind farm-1, results obtained for wind farm-2 are similar.

- Probabilities of each of the three scenarios will be calculated based on Chapter 3 .

1. $V_{C I} \leq V \leq V_{R}$ : Wind farms 1 and 2 are fed with a wind speed of $6 \mathrm{~m} / \mathrm{s}$ between the cut-in speed and the rated speed. The speed is kept constant at $6 \mathrm{~m} / \mathrm{s}$.

Figure 5.9 shows the output of the wind farm-1. It can be noted that when the wind speed is below the rated wind speed, the output is very small. It only produces about 1.4MW (accrding to analytical computations it should be around $1.71 \mathrm{MW}$ ) out of its total capacity of $18 \mathrm{MW}$. No pitch angle control is needed at this point and the pitch angle remains zero. 

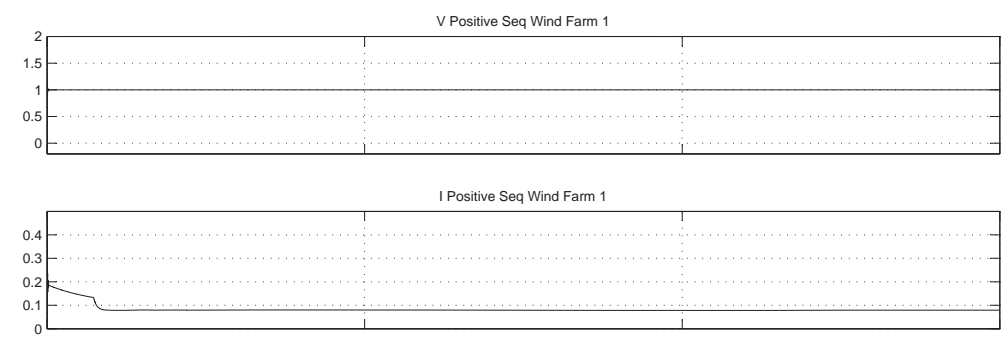

P_mean (MW

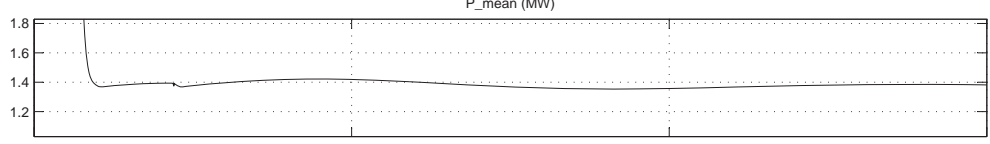

Q_mean (MW
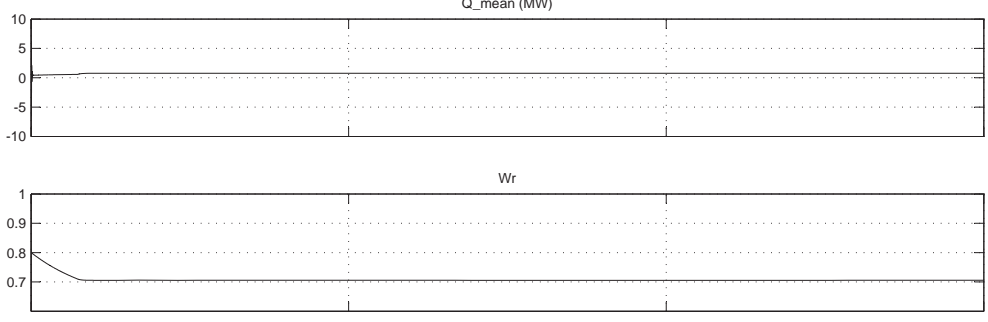

Pitch_Angle

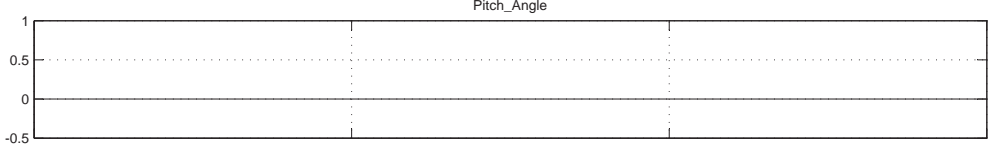

Wind Speed

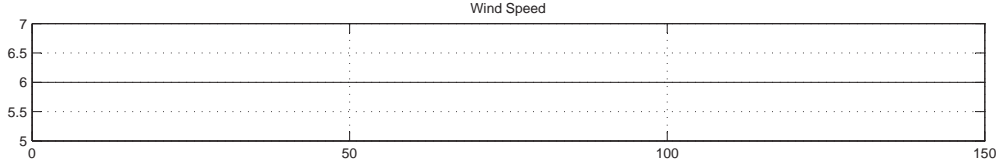

Time offset: 0

Figure 5.9 - Wind Farm-1 Output at $V=6 \mathrm{~m} / \mathrm{s}$

2. $V=V_{R}$ : Wind farms-1 and 2 are fed with a wind speed of $14 \mathrm{~m} / \mathrm{s}$, which is the rated speed for the turbine model. The speed is slowly increased from $6 \mathrm{~m} / \mathrm{s}$ to $14 \mathrm{~m} / \mathrm{s}$ at a rate of $0.1 \mathrm{~m} / \mathrm{s}^{2}$.

Figure 5.10 shows the output of wind farm-1 at rated wind speed. As seen from the figure, the output is the rated output of almost 18MW. At this point, the pitch angle control of the wind turbine model slowly starts to kick-in, resulting in slight movement of $1^{\circ}$. 

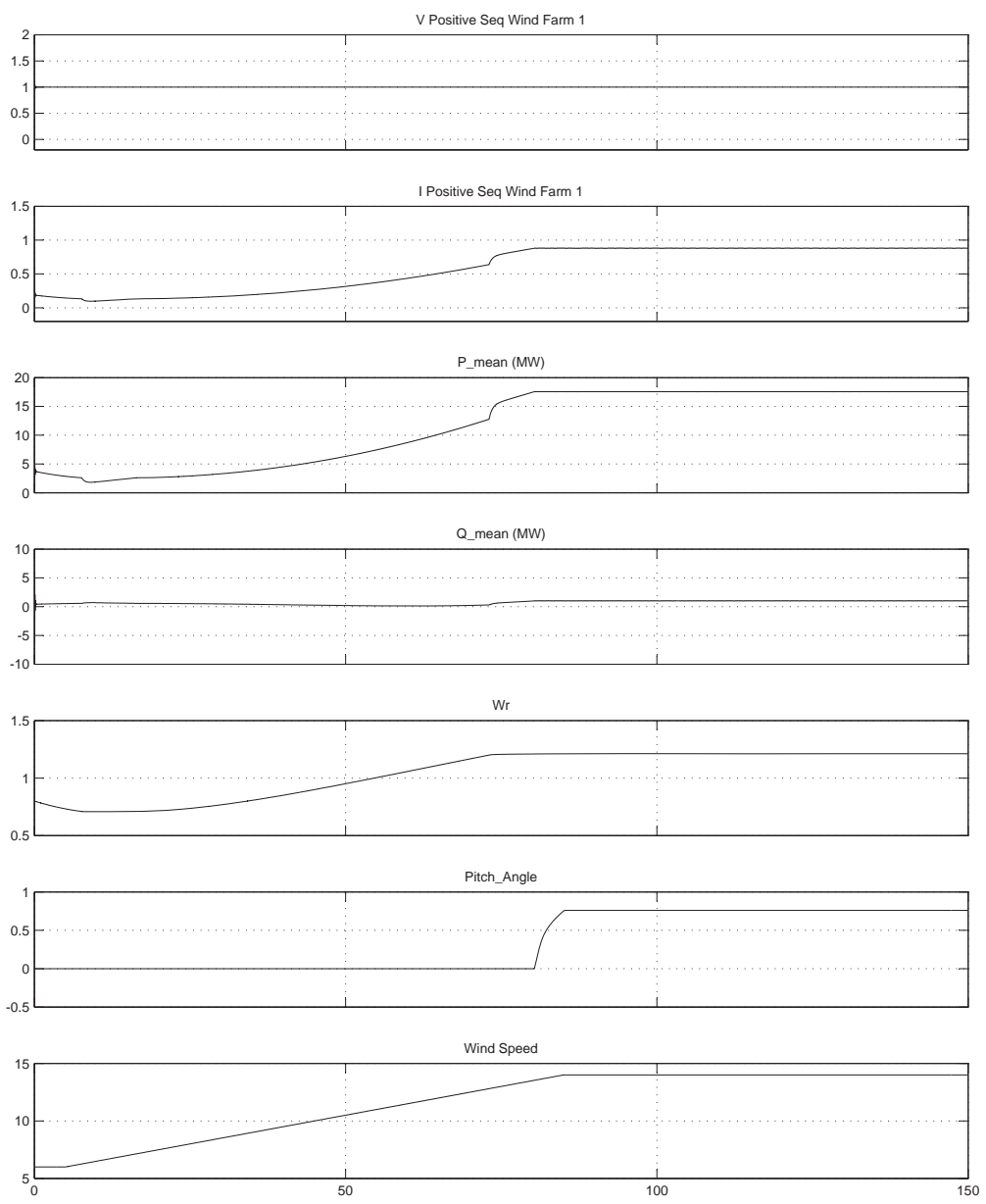

Time offset: 0

Figure 5.10 - Wind Farm-1 Output at $V=14 \mathrm{~m} / \mathrm{s}$

3. $V_{R} \leq V \leq V_{C O}$ : Wind farms-1 and 2 are fed with a wind speed of $22 \mathrm{~m} / \mathrm{s}$ between the rated speed and the cut-off speed. The speed is increased at the same rate of $0.1 \mathrm{~m} / \mathrm{s}^{2}$ from $6 \mathrm{~m} / \mathrm{s}$ to $22 \mathrm{~m} / \mathrm{s}$.

Figure 5.11 shows the output of the wind farm- 1 at a speed of $22 \mathrm{~m} / \mathrm{s}$, which is much above the rated speed of the wind turbine, but not close to cut-off speed yet. It can be noted that the output is still the rated output. The pitch angle control, however, starts increasing significantly, increasing almost up to $20^{\circ}$. 

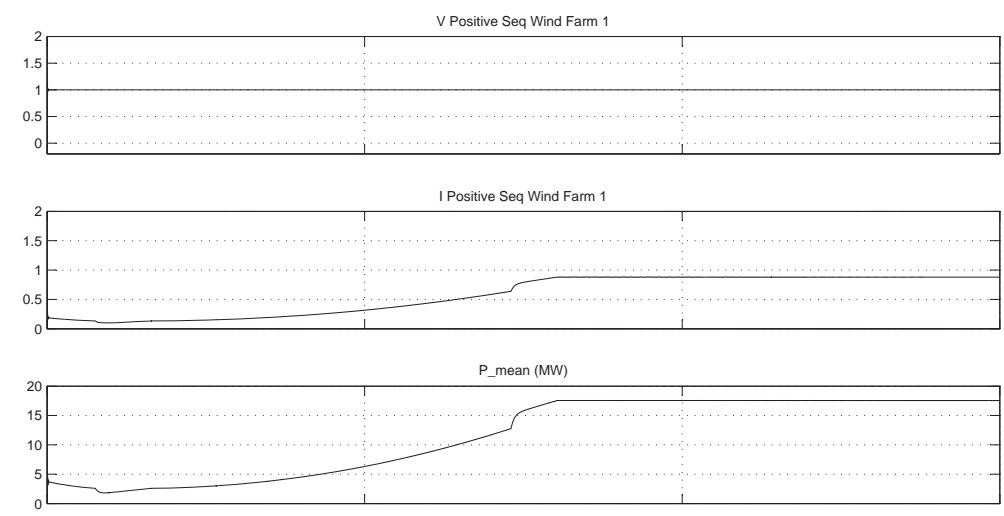

Q_mean (MW)
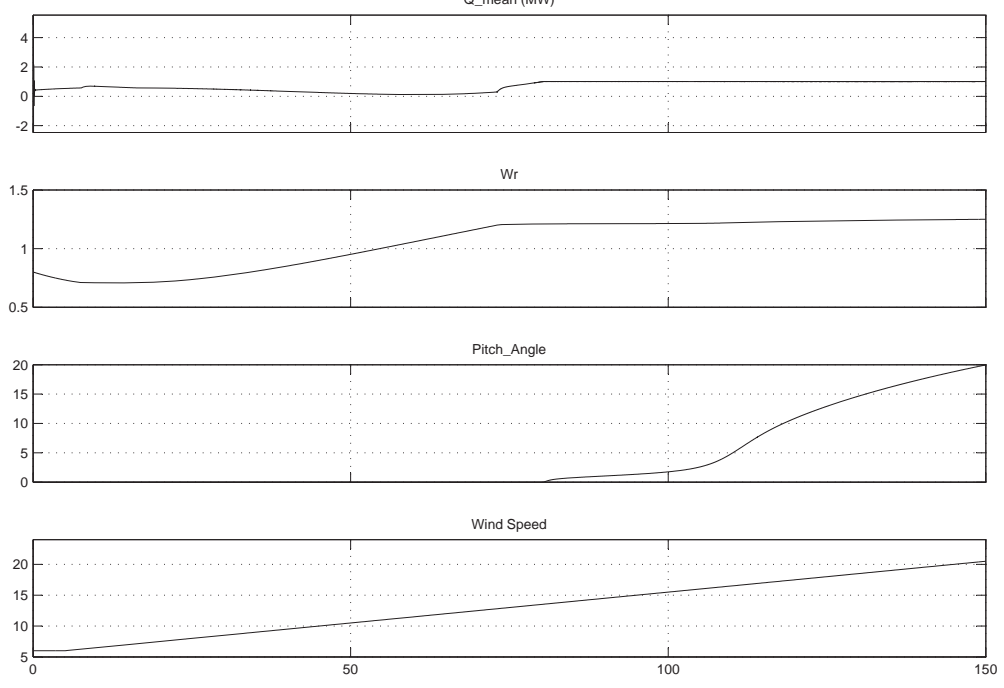

Time offset: 0

Figure 5.11 - Wind Farm-1 Output at $V=22 \mathrm{~m} / \mathrm{s}$

In case 1 , for very low outputs from wind turbines, default switching combination is the best, since the total load at both the feeders is much more than the output from wind generation. Feeders DS1 and DS3 have total loads of 15MW and 22.5MW respectively and when the turbines are producing less then $10 \%(1.4 \mathrm{MW}$ and $0.17 \mathrm{MW})$ of the total load, the default condition where all of the load is supplied by the grid is preferred. However, once the wind farms start producing about $50 \%$ of the total power (wind farm1 produces around 9MW and wind farm-2 about 4.5MW), switching combinations 3 and 5 (See Figure 5.3) can be used under normal operating conditions. 
In cases 2 and 3, assuming a uniform speed over the region, wind farm- 1 is producing close to $18 \mathrm{MW}$ and wind farm-2 is producing $9 \mathrm{MW}$. A better switching combination for these cases is combination 3 (See Figure 5.3). In this case, the wind farm-1 is supplying half of feeder DS-1 and the left over is being used to support DS-2.

Next section will calculate the probabilities of each of the three scenarios. The scenario where output will be zero is also covered. It should be noted that in an event of zero output from wind turbines, default combination (See Figure 5.3) should be used.

\section{Probabilities of Different Outputs}

It is of interest to know the probability of each of the above scenarios. The most important factor in the three scenarios is the wind speed. Probabilities of individual wind speeds for each hour have already been calculated (See Chapter 3). Based on the probabilities of the individual wind speeds, it is possible to calculate the probabilities of different levels of outputs from the wind farms.

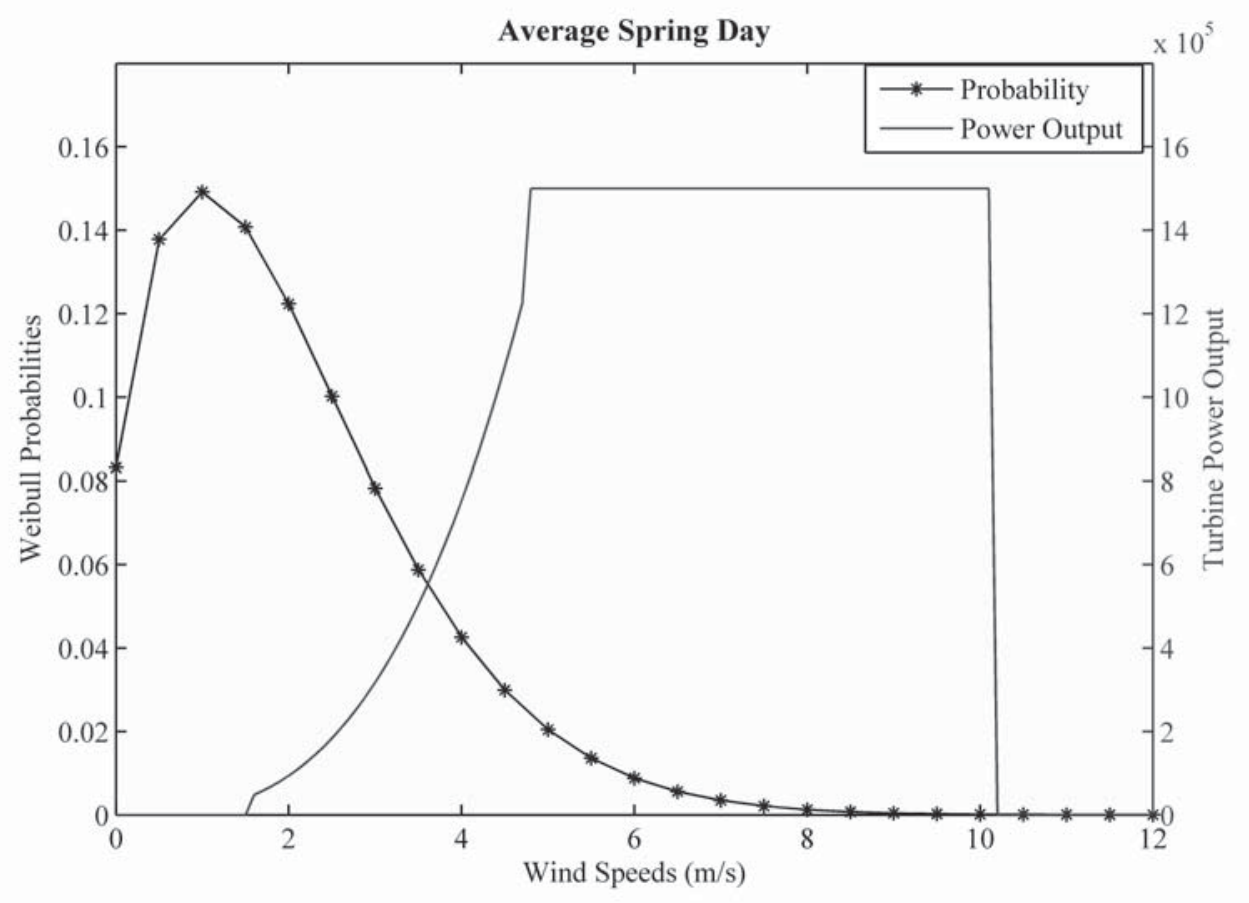

Figure 5.12 - Wind Speed Probabilities and Relevant Output 
Table 5.1

Cumulative Weibull Probabilities

\begin{tabular}{llllll}
\hline Scenario & Spring & Summer & Fall & Winter & Output \\
\hline Below cut-in & 0.393574 & 0.630765 & 0.570638 & 0.416845 & $0 \mathrm{MW}$ \\
Between cut-in and rated & 0.544834 & 0.358901 & 0.396134 & 0.519203 & $0-1.5 \mathrm{MW}$ \\
Between rated and cut-off & 0.061275 & 0.009931 & 0.032302 & 0.063398 & $1.5 \mathrm{MW}$ \\
Above cut-off & 0.000223 & 0.000005 & 0.000207 & 0.000379 & $0 \mathrm{MW}$ \\
\hline
\end{tabular}

Table 5.2

Cumulative Nonparametric Probabilities

\begin{tabular}{llllll}
\hline Scenario & Spring & Summer & Fall & Winter & Output \\
\hline Below cut-in & 0.3791 & 0.6087 & 0.5454 & 0.4174 & $0 \mathrm{MW}$ \\
Between cut-in and rated & 0.5781 & 0.3883 & 0.4247 & 0.523 & $0-1.5 \mathrm{MW}$ \\
Between rated and cut-off & 0.0418 & 0.003 & 0.0298 & 0.0596 & $1.5 \mathrm{MW}$ \\
Above cut-off & 0.0009 & 0 & 0 & 0 & $0 \mathrm{MW}$ \\
\hline
\end{tabular}

Figure 5.12 shows the Weibull probabilities calculated for an hour chosen randomly (mid-night to 1:00AM) on a typical spring day. The output curve of one of the $1.5 \mathrm{MW}$ turbines used for simulation is overlapped on the probability curve for a better perspective. It should be noted that the wind speeds on the output curve have already been adjusted for the hub height (See Equation (4.3)) of the wind turbine.

Tables 5.1 and 5.2 show Parametric (Weibull in this case) probabilities and nonparamtric probabilities calculated for the same hour of the day for each season. The methodology to do this has already been described in Chapter 3. The following is easily observed:

- There is significant difference in probabilities of different season. Probability of any output being the lowest in Summer for both parametric and nonparametric approaches.

- For the data set of wind speeds under scrutiny, it is observed that the probability of rated output is very low, making this wind site less desirable.

- It should also be noted that since the nonparametric calculation only focuses on the data set, it has assigned zero probability to many speeds above the cut-off, which may not be the real case. 
- The Weibull probabilities are more conservative compared to the nonparametric probabilities.

- As for the Weibull probability, since it follows a Weibull density, even the speeds beyond cut-off have been assigned a small probability.

Probabilities thus calculated can be used to anticipate the probabilities of power outputs of different level for any given location.

\subsubsection{Emergency Conditions}

The most important thing under emergency operating condition to isolate the fault first. As indicated earlier, considering all of the possible combinations of various types of faults at different locations in the test system is beyond the scope of the thesis. However, the three main emergency conditions that wind generation directly affects are considered. The purpose of the following results is to show the load flow results in both high and low outputs from the wind farms in the scenarios discussed in Section 5.2.3. Note that the switches with '?' are considered to be 1 in the following simulation results.

\section{Outage at Feeder DS-1 Supply:}

The wind farm connected to Feeder DS-1 is Wind farm-1, with a capacity of $18 \mathrm{MW}$. Generation outage at DS-1 also means that the grid support A.7 for wind farm-1 is lost. However, in this case, the wind farm can still have grid support by enabling the tie switch 3 (See Figure 5.2). Total load on DS-1 is $15 \mathrm{MW}$, that means that wind farm- 1 can supply all of it, given it is producing its rated output. In case of a lower output of $50 \%$ from the wind farm, $60 \%$ of the load on DS-1 can still be provided. 

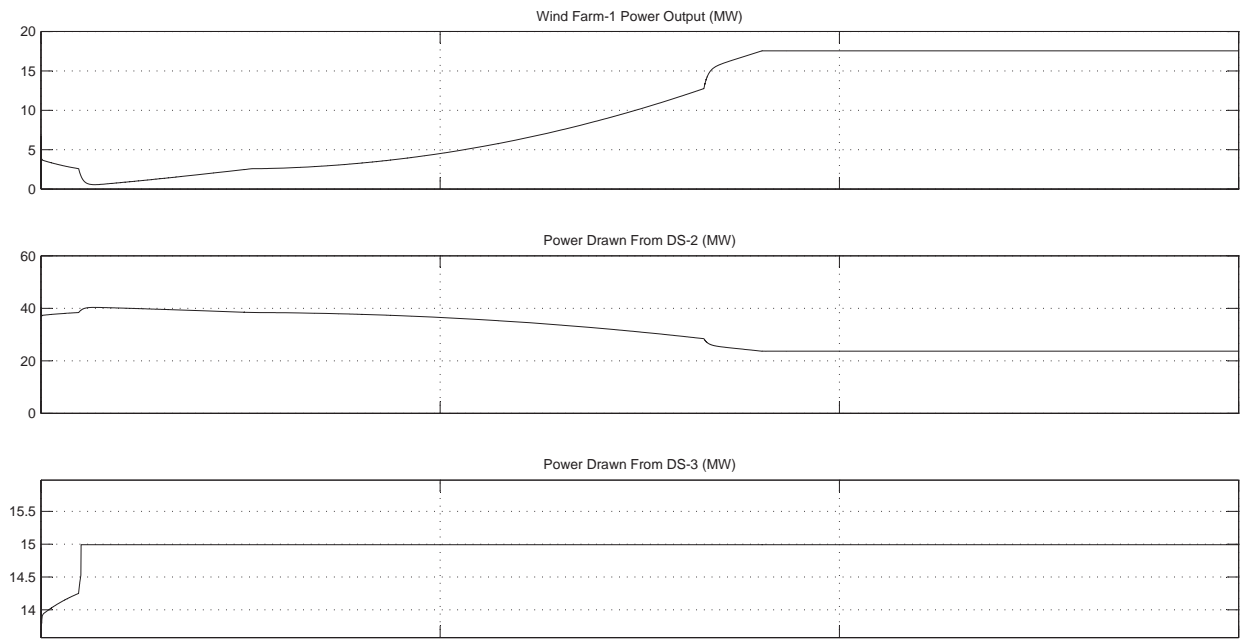

DS-1 Power (MW)

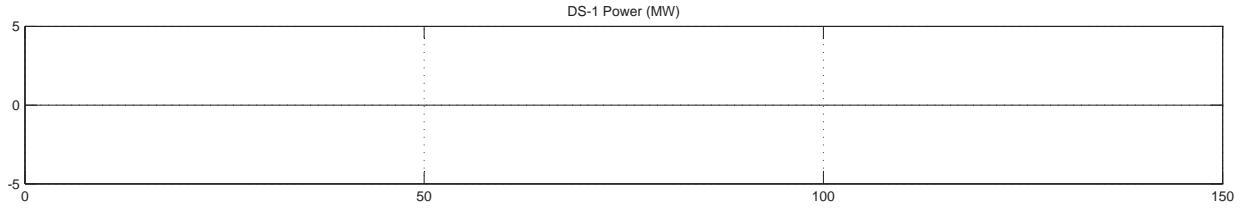

Time offset: 0

Figure 5.13 - DS-1 Outage: Full Output from Wind Farms-1 and 2

Figure 5.13 shows the result for the combination on the left side of Figure 5.6. It is clear that in an event when the wind farm-1 is producing anywhere above $75 \%$ of its capacity (wind speeds of $5 \mathrm{~m} / \mathrm{s}$ and above on ground), the wind farm- 1 is capable of supplying all of the load on DS-1. In this case, the grid support is provided by DS-2 via switch 3 . Note that the total load on feeder DS-3 is $22.5 \mathrm{MW}$ but since the wind farm- 2 is also producing at its rated capacity, DS-2 only has to supply the remainder. 

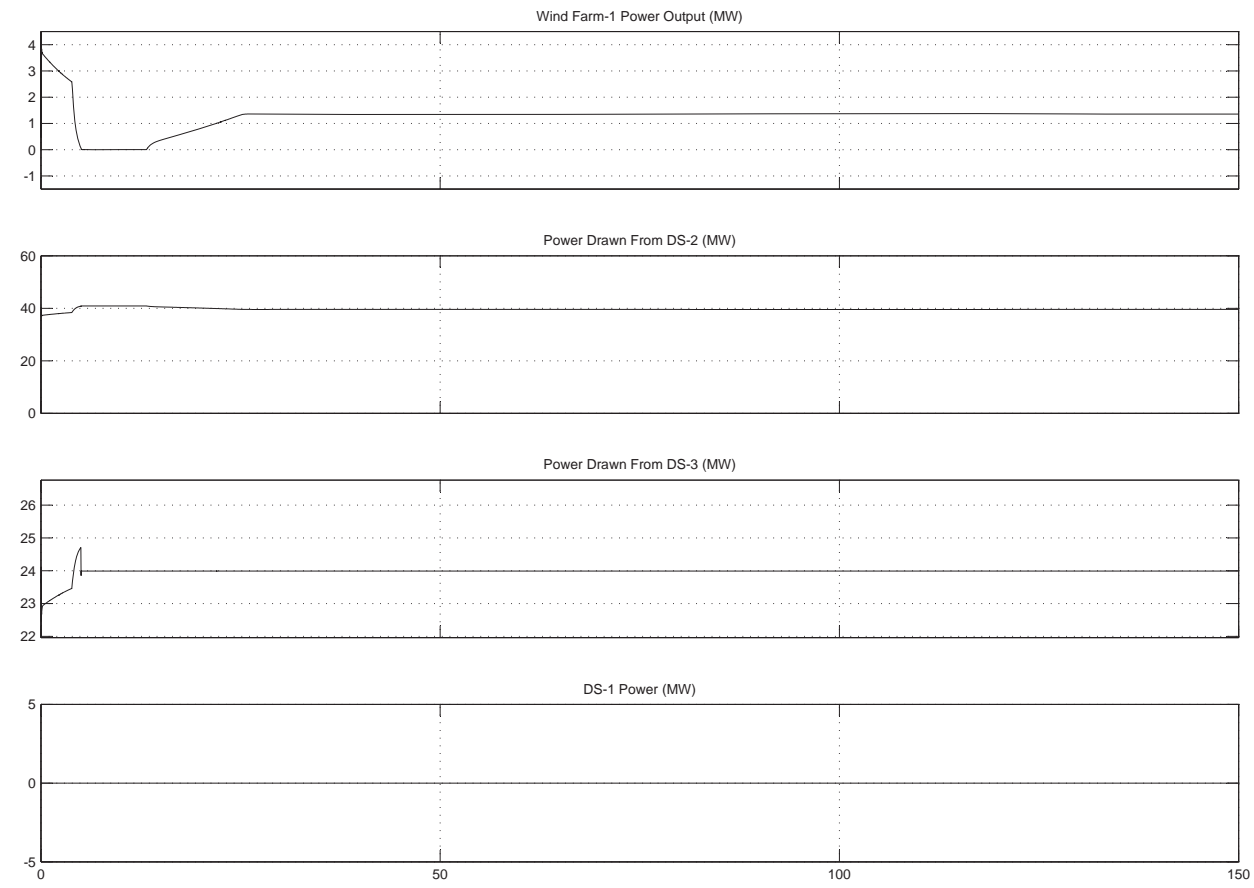

Figure 5.14 - DS-1 Outage: Low Output from Wind Farms-1 and 2

Figure 5.14 shows an output in case of low wind speeds ( $\leq 3 \mathrm{~m} / \mathrm{s}$ on the ground). The speed considered here is $2.3 \mathrm{~m} / \mathrm{s}$ on ground (about $6 \mathrm{~m} / \mathrm{s}$ at the hub). In this case, the switching topology shown on the right side of Figure 5.6 is used. This is still a valid topology. However, it must be noted that the power drawn from DS-2 in this case is almost 40MW, which is about 10MW more then its total load. In practice, the feeder may not have this much excess capacity and it is evident that some of the load in this case would have to be curtailed.

\section{Outage at Feeder DS-2 Supply:}

There is no wind farm directly connected to feeder DS-2, however, both wind farms-1 and 2 can be connected to DS- 2 indirectly by operation of the tie switches 3 and 5 . Keeping in mind that the network must remain radial, sectionalizing switch 4 must be opened 
(See Figure 5.3). DS-2 has a total of $30 \mathrm{MW}$ connected to it. This is more then the total capacity of both the wind farms- 1 and 2 combined, which is $18 \mathrm{MW}+9 \mathrm{MW}=27 \mathrm{MW}$. Evidently, the probability that all of the load on DS-2 be supplied by wind generation is zero. However, the indirect connection to wind farms- 1 and 2 does mean that in an event of rated output from the wind farms, $90 \%$ of the load on DS-2 can be supplied by wind.
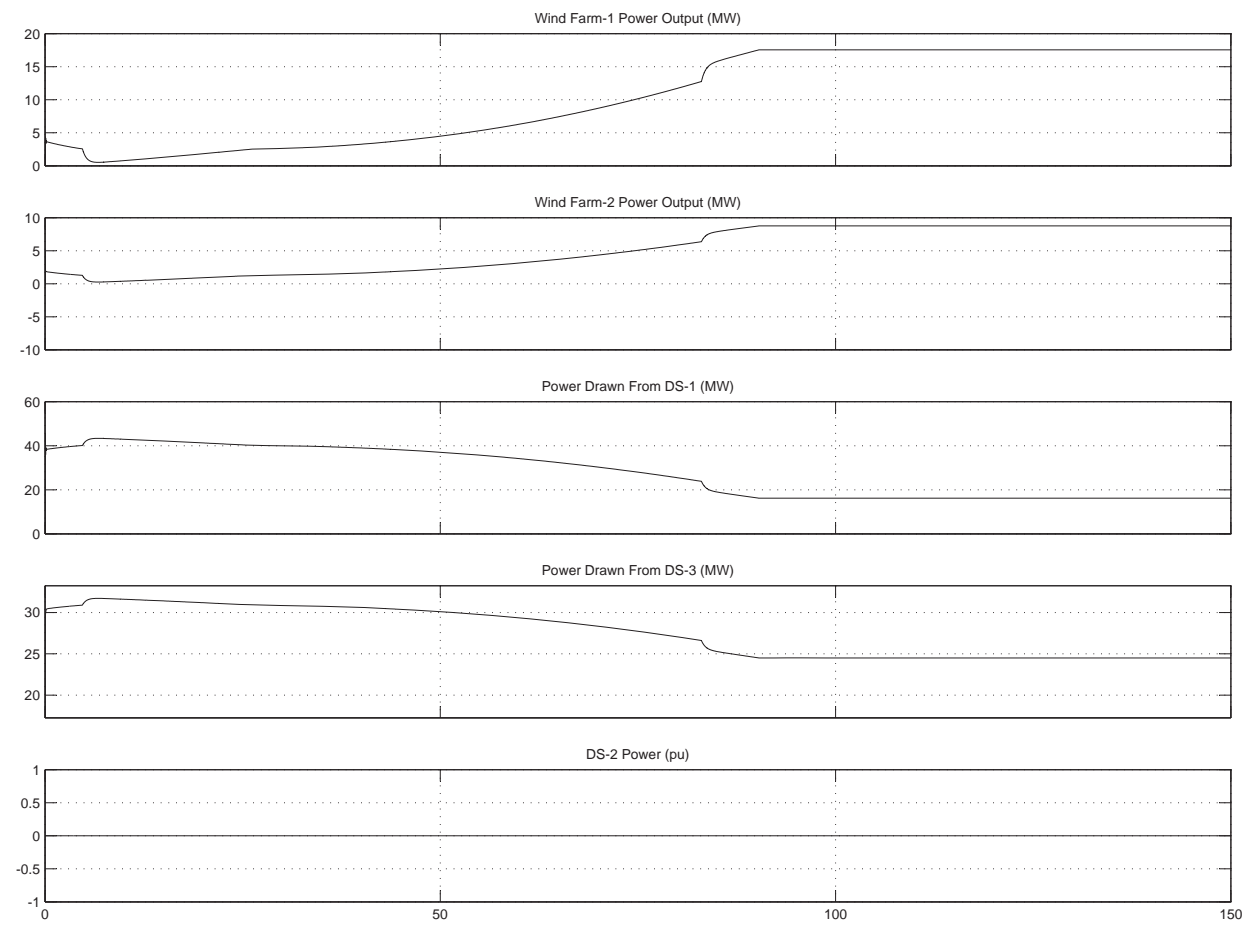

Time offset: 0

Figure 5.15 - DS-2 Outage: Full Output from Wind Farms-1 and 2

As indicated in the paragraph above, there is no way that $100 \%$ of load on the DS2 feeder can be supplied by wind generation. However, Figure 5.15 shows a scenario when both the wind farms in the system are operating at their rated capacity. In this case, the deficit is supplied by the two feeders DS-1 and DS-3. 

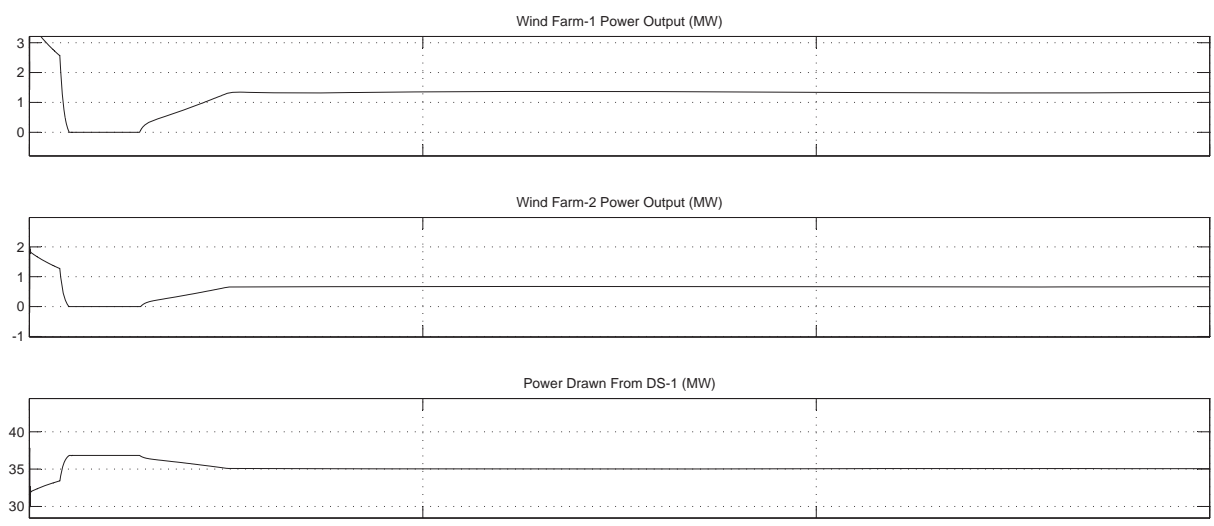

Power Drawn From DS-3 (MW)
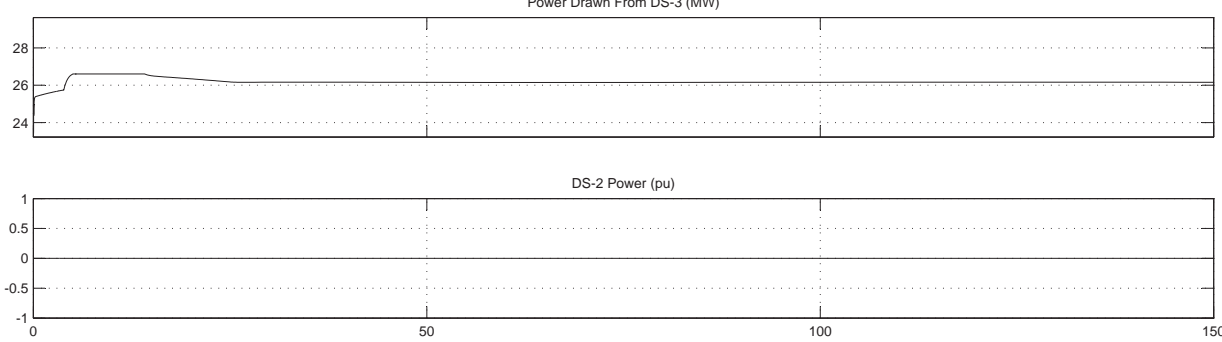

Time offset: 0

Figure 5.16 - DS-2 Outage: Low Output from Wind Farms-1 and 2

Figure 5.16 shows a scenario when the output from the wind farms is very low (same $6 \mathrm{~m} / \mathrm{s}$ as above). It is seen that the power drawn from feeders DS-1 and DS-3 is extremely high (35MW and 26MW respectively). The reason for choosing the same network topology as that used on the left side of the Figure 5.7 is that for any other combinations, loops will be introduced in the topology. This goes on to show that in case of a lower output from the wind farms, some of the load will remain unserved, if a large capacity on feeders DS-1 and DS-3 is not available.

\section{Outage at Feeder DS-3 Supply:}

Wind farm-2 with a rated capacity of $9 \mathrm{MW}$ is connected to DS-3, which has $22.5 \mathrm{MW}$ of load. In case of failure of generation at this bus, wind farm-1 by itself is insufficient. In this case, an attempt to use the output from wind farm-1 can be made by operation of tie 
switches 3 and 6. In an event of rated output from the wind farms, $90 \%$ of the load on the left side of switch 7 (See Figures 5.2 and 5.8) can be supplied by wind generation at wind farm-2. Output from wind farm-1 can support almost 100\% the load on right side of switch 7. In case of an output lower then 50\%, for the same switching combination, the load on the left side of switch 7 may remain unserved.
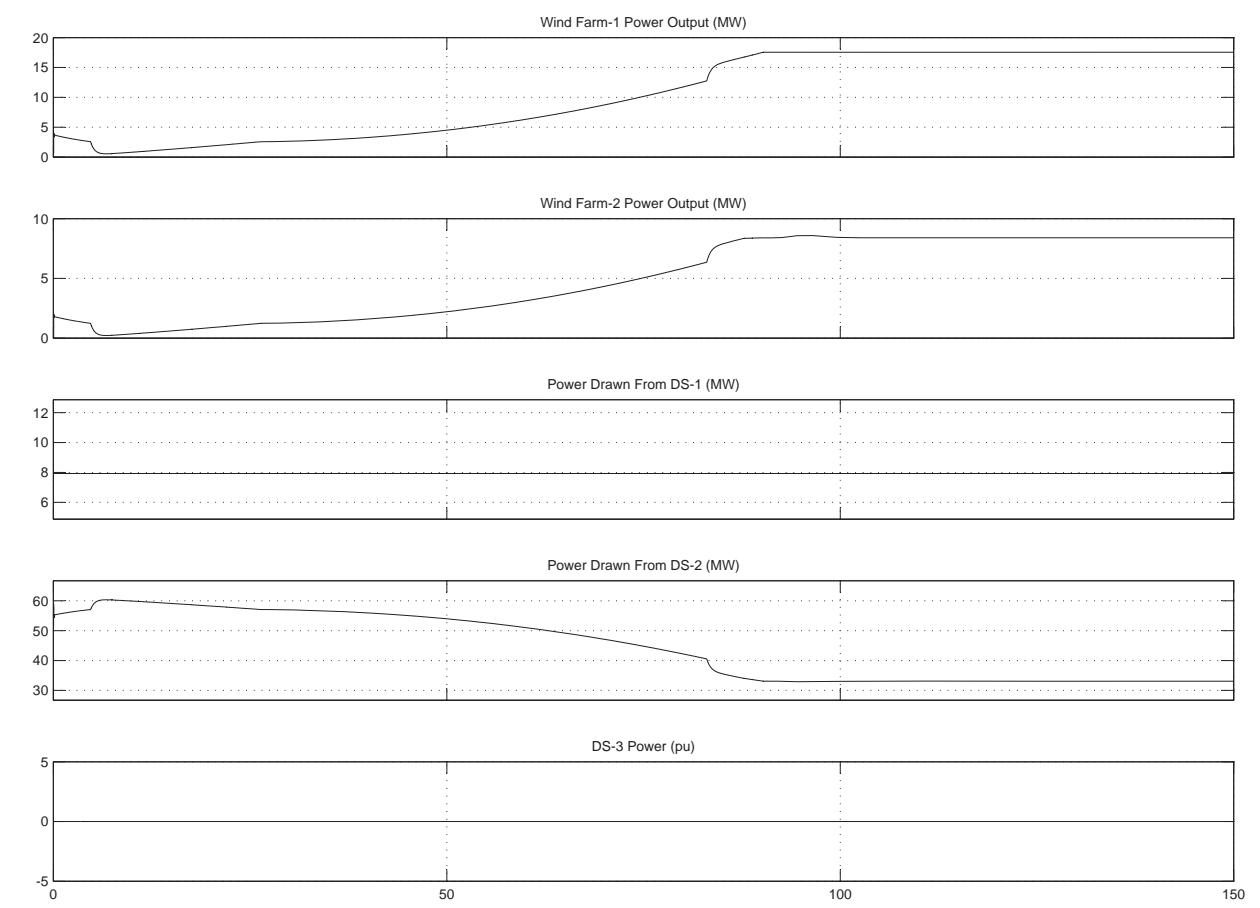

Time offset: 0

Figure 5.17 - DS-3 Outage: Full Output from Wind Farms-1 and 2

Figure 5.15 shows the topology used on the left side of the Figure 5.8. In this case, the wind farm-2 supplies all of its 9MW to DS-3 load. The grid support is provided by DS-2 with switch 6. Wind farm-1 is capable of supplying the 7MW on the right side of the switch 1. The remainder of its output(10MW) is indirectly fed to the load on DS-3. In addition to the output from wind farms-1 and $2(10 \mathrm{MW}+9 \mathrm{MW})$, the feeder DS-2 has to supply the remaining $3.5 \mathrm{MW}$ of load on DS-3. 

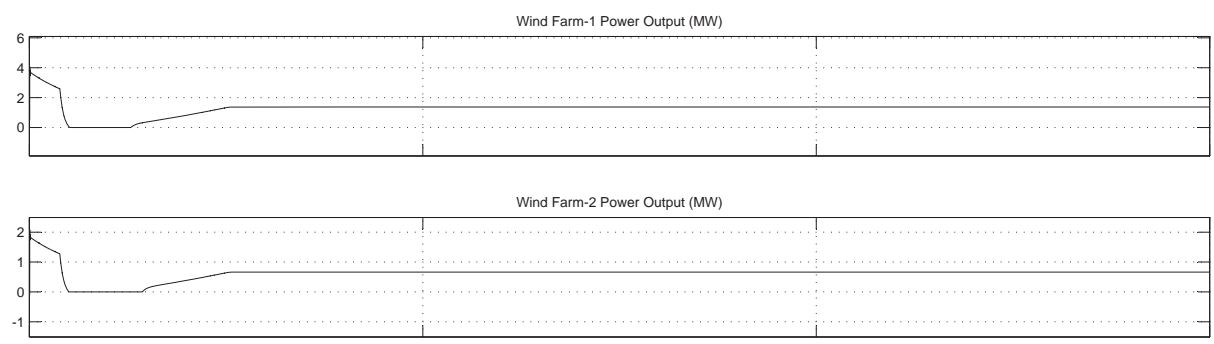

Power Drawn From DS-1 (MW)

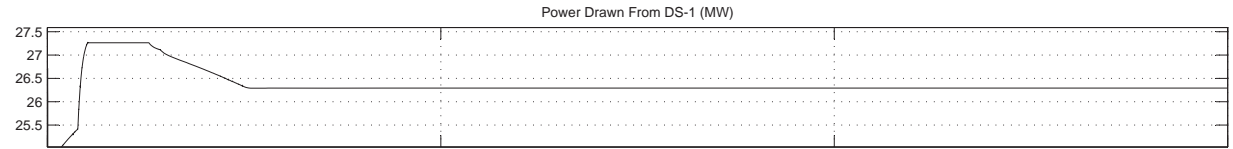

Power Drawn From DS-2 (MW)
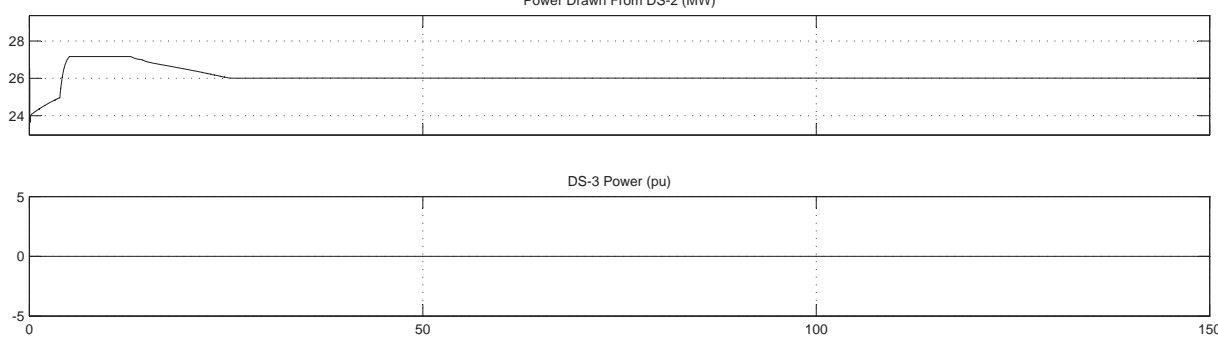

Time offset: 0

Figure 5.18 - DS-3 Outage: Low Output from Wind Farms-1 and 2

Figure 5.18 shows the topology on the right side of Figure 5.8. In this case, the wind farms are not producing any significant output and as a result, the feeder DS-2 has to be overloaded to almost twice its capacity in order to provide the load on DS-3. In summary, the following should be recognized for the results discussed above:

- When wind turbines are producing at their maximum, they can provide for feeders DS-1 and DS-3.

- In case of a low output, the other two feeders in all three cases have to be heavily loaded. This may not be feasible in practice and hence the right sides of each of the Figures 5.6, 5.7, and 5.8 show that some of the load may be unserved in an event of low output from the wind farms combined with a small excess capacity of each of the healthy feeders. 
Table 5.3

Cumulative Weibull Probabilities for Loss of Generation in Each Feeder

\begin{tabular}{|c|c|c|c|c|c|c|c|}
\hline \multirow{2}{*}{ Feeder } & \multirow{2}{*}{ Total Load } & \multirow{2}{*}{$\begin{array}{l}\% \text { Load } \\
\text { Supplied } \\
\text { by Wind }\end{array}$} & \multirow{2}{*}{$\begin{array}{l}\text { Load in } \\
\text { MW }\end{array}$} & \multicolumn{4}{|c|}{ Weibull Probabilities } \\
\hline & & & & Spring & Summer & Fall & Winter \\
\hline \multirow{3}{*}{ DS-1 } & \multirow{3}{*}{$15 \mathrm{MW}$} & $0 \%$ & 0 & 0.3938 & 0.6308 & 0.5708 & 0.4172 \\
\hline & & $<50 \%$ & $<7.5$ & 0.34659 & 0.3319 & 0.4538 & 0.4326 \\
\hline & & $100 \%$ & 15 & 0.0733 & 0.0129 & 0.0387 & 0.0750 \\
\hline \multirow{3}{*}{ DS-2 } & \multirow{3}{*}{$30 \mathrm{MW}$} & $0 \%$ & 0 & 0.3938 & 0.6308 & 0.5708 & 0.4172 \\
\hline & & $<50 \%$ & $<15$ & 0.4355 & 0.3244 & 0.3357 & 0.4154 \\
\hline & & $100 \%$ & 30 & 0.00 & 0.00 & 0.00 & 0.00 \\
\hline \multirow{3}{*}{ DS-3 } & \multirow{3}{*}{$22.5 \mathrm{MW}$} & $0 \%$ & 0 & 0.3938 & 0.6308 & 0.5708 & 0.4172 \\
\hline & & $<50 \%$ & $<11.25$ & 0.4085 & 0.3124 & 0.3192 & 0.3899 \\
\hline & & $100 \%$ & 22.5 & 0.1104 & 0.0239 & 0.0590 & 0.1105 \\
\hline
\end{tabular}

\section{Relevant probabilities}

In an event of a loss of generation at any of the three feeders, it is of interest to anticipate the amount of load that can be still served by wind. In order to do this, probabilities of output from wind are calculated for the same hour that was considered in Tables 5.1 and 5.2. Feasible switching combinations for loss of generation scenarios have already been discussed in Section 5.6. This section will tabulate the probability of load being supplied by wind generation in each of the three scenarios.

Table 5.3 shows the probabilities of output from wind generation in the test system. Tables 5.3 and 5.4 show cumulative Weibull and nonparametric probabilities of various outputs from wind generation for the same time-frame of 12:00 midinght to 1:00 AM. The following should be noted in reagrds to Tables 5.3 and 5.4:

- Probabilities of no output are higher in summer and fall seasons, whereas, those of a rated output are higher in spring and winter season.

- Probability of $15 \mathrm{MW}$ output in case of outage at DS-1 is significantly lower then that calculated in case of an outage on DS-2. This is owing to the fact that DS-1 is directly associated to only wind farm-1 and hence the probabilities calculated are for all of the $15 \mathrm{MW}$ coming from wind farm-1 only. Whereas, in case of an outage at DS-2, both of the wind farms- 1 and 2 can be connected indirectly to 
Table 5.4

Cumulative Nonparametric Probabilities for Loss of Generation in Each Feeder

\begin{tabular}{|c|c|c|c|c|c|c|c|}
\hline \multirow{2}{*}{ Feeder } & \multirow{2}{*}{ Total Load } & \multirow{2}{*}{$\begin{array}{l}\% \text { Load } \\
\text { Supplied } \\
\text { by Wind }\end{array}$} & \multirow[t]{2}{*}{$\begin{array}{l}\text { Load in } \\
\text { MW }\end{array}$} & \multicolumn{4}{|c|}{ Nonparametric Probabilities } \\
\hline & & & & Spring & Summer & Fall & Winter \\
\hline \multirow{3}{*}{ DS-1 } & \multirow{3}{*}{$15 \mathrm{MW}$} & $0 \%$ & 0 & 0.38 & 0.6087 & 0.5454 & 0.4174 \\
\hline & & $<50 \%$ & $<7.5$ & 0.4686 & 0.3670 & 0.3788 & 0.4296 \\
\hline & & $100 \%$ & 15 & 0.0538 & 0.0041 & 0.0370 & 0.0718 \\
\hline \multirow{3}{*}{ DS-2 } & \multirow{3}{*}{ 30MW } & $0 \%$ & 0 & 0.38 & 0.6087 & 0.5454 & 0.4174 \\
\hline & & $<50 \%$ & $<15$ & 0.4451 & 0.3586 & 0.3714 & 0.4123 \\
\hline & & $100 \%$ & 30 & 0.00 & 0.00 & 0.00 & 0.00 \\
\hline \multirow{3}{*}{ DS-3 } & \multirow{3}{*}{$22.5 \mathrm{MW}$} & $0 \%$ & 0 & 0.38 & 0.6087 & 0.5454 & 0.4174 \\
\hline & & $<50 \%$ & $<11.25$ & 0.4137 & 0.3475 & 0.3582 & 0.3865 \\
\hline & & $100 \%$ & 22.5 & 0.0961 & 0.0099 & 0.0541 & 0.1068 \\
\hline
\end{tabular}

DS-2 using combinations discussed in 5.2.3. This implies that the probabilities calculated in this case are the probabilities of the cumulative output of both the wind farms.

- Probabilities of wind farms supplying all of the load in case of an outage at DS-2 are 0 for all seasons. This is due to the fact that the total rated capacity of both the wind farms is $18 \mathrm{MW}+9 \mathrm{MW}=27 \mathrm{MW}$, which is less then the load connected at DS-2 (30MW).

Probabilities for all 24 hours and their associated outputs are calculated and tabulated in Table A.2.

\subsection{Summary}

This chapter incorporated the wind speed probabilities and the associated wind power output into a small test system. Appropriate switching combinations were defined and identified for this test system. The tasks completed in this chapter are summarized as follows:

1. Distribution system reconfiguration using remote controllable switches was discussed. 
2. Elementary feasibility constraints for both normal and emergency operating conditions were identified and listed.

3. Constraints were applied to a small test system with wind generation and evaluated.

4. Appropriate switching combinations under normal operating conditions were listed.

5. Three possible loss-of-generation scenarios were discussed and appropriate switching actions were suggested

6. Probabilities of different power outputs were calculated and relevant switching combinations were suggested. 


\section{Chapter 6}

\section{Results and Future Work}

The aim of this work was three-fold: (1) calculate probabilities of all wind speeds that occurred in a given time-frame, (2) use the calculated probability and obtain average predicted power, and (3) identify feasible switching combinations for different levels outputs and their relevant probability. In essence, a list of possible normal and emergency scenarios in presence of wind generation and their respective probabilities are desired. This chapter summarizes this thesis and provide recommendation for future work.

\subsection{Major Research Contributions}

The goal of this work is to calculate and apply wind speed probabilities, both analytically and empirically to a medium voltage test system in order to identify various possible switching topologies. To achieve this, the following tasks were accomplished:

- Analysis of historical wind speed data of 4 years was performed. Based on the analysis, two different approaches to calculate probability were used. Parametric statistics uses Weibull probability density function to obtain probabilities of all of the wind speeds observed in the past. The nonparametric method calculates probabilities of various wind speeds based purely on the data set available. Most likely wind speeds are obtained using the calculated probabilities. Probabilities calculated from both of these methods were also compared. 
- Incorporating the wind speed probabilities into power output calculation was the second task. For this, first, various factors affecting the output of a wind turbine were discussed and power output of a typical 1.5MW wind turbine was calculated. Using the probabilities calculated earlier, average hourly output was obtained.

- Finally, using a small test network, a number of possible scenarios were analyzed and their relevant probabilities were calculated. First, a set of feasibility constraints were identified and listed. These constraints were applied to the possible topologies in test system. After removing the nonfeasible combinations, the remaining were tested for different levels of output from wind generation. Probabilities relevant to the different levels of wind generation were then calculated.

\subsection{Future Work}

The following relevant research topics are of importance and deserve further investigation:

1. Various Types of DG: This work solely focuses on wind DG. The next logical step is to include more types of DG into the system. Specifically:

- Secondary distribution systems can accommodate solar thermal and PV applications more easily than wind. Modeling of solar arrays will be rather challenging, but beneficial. Stochastically modeling the solar irradiance and influencing factors such as clouds and diffracting particles in the atmosphere will be interesting.

- Other sources, such as bio-mass and fuel-cells, they can be later included into the model to make it more complete. Especially given the non-intermittent nature of fuel-cells and bio-mass can be combined with intermittent sources to solve an optimal allocation of conventional and non-conventional sources.

- Load variations were not considered in this work, since it is a short-term prediction problem. For longer time-frames, inclusion of variability of loads can be considered.

2. Wind Power Predictions: A mathematical model that improves on the assumptions made here is bound to give interesting results. Use of other methods like 
time series models and neural networks for wind speed predictions for a smaller system can be of interest. Later, the comparison of this work to the results from these models above can be included in future work.

- This work used calculated probabilities of various wind speeds, which was based purely on the frequency of appearance of a particular wind speed for a given time-frame. In order to improve the representation of time into the analysis, the next step in this direction would be to use conditional probability in order to include time into the analysis.

- The wind speed data is periodic on a short-term diurnal as well as on a long term seasonal or annual basis. The time averaging models may be able to define periodicity of the data accurately.

3. Optimal Switching Operations for Large Distribution Systems: For largescale urban systems, more mathematically complex models are required. Developing methodologies for this purpose should be the next step in the process.

4. Protection Coordination: Protection coordination is one of the main reasons for the popularity of a radial topology. Protection schemes that take the addition of DG into account will be useful.

5. Switching Transients: This work focused on the steady-state results obtained by switching actions. Transients generated from switching actions were beyond the scope of the work. A study of transient effects of switching actions should be a future step. 


\section{Bibliography}

[1] G. Heydt, "The next generation of power distribution systems," Smart Grid, IEEE Transactions on, vol. 1, pp. 225 -235, December 2010.

[2] E. Coster, J. Myrzik, B. Kruimer, and W. Kling, "Integration issues of distributed generation in distribution grids," Proceedings of the IEEE, vol. 99, pp. $28-39$, January 2011.

[3] J. Guerrero, F. Blaabjerg, T. Zhelev, K. Hemmes, E. Monmasson, S. Jemei, M. Comech, R. Granadino, and J. Frau, "Distributed generation: Toward a new energy paradigm," Industrial Electronics Magazine, IEEE, vol. 4, pp. 52 -64, March 2010.

[4] A. Sundaram, "Power quality impacts of distributed generation: Survey of distributed generation technologies," EPRI, pp. 11 -60, 2000.

[5] "IEEE application guide for IEEE std 1547, 'IEEE standard for interconnecting distributed resources with electric power systems," IEEE Std 1547.2-2008, pp. 1 -207, 152009.

[6] C. J. Mozina, "A tutorial on the impact of distributed generation on distribution systems," in Proc. thst 61st Protective Relay Engineers, (College Station, TX), pp. 591-609, Apr. 1-3 2008.

[7] R. Walling, R. Saint, R. Dugan, J. Burke, and L. Kojovic, "Summary of distributed resources impact on power delivery systems," Power Delivery, IEEE Transactions on, vol. 23, pp. $1636-1644$, july 2008. 
[8] B. Hamilton and M. Summy, "Benefits of the smart grid [in my view]," Power and Energy Magazine, IEEE, vol. 9, pp. 104 -102, Jan.-Feb. 2011.

[9] A. Ipakchi and F. Albuyeh, "Grid of the future," Power and Energy Magazine, IEEE, vol. 7, pp. 52 -62, March-April 2009.

[10] "Hirlam - high resolution limited area model." http://hirlam.org/ index $\cdot$ php?option=com_content \&view=category\&layout $=$ $b \log \& i d=52 \& I t e m i d=53$.

[11] "Mesoscale model, pennsylvenia state university." http: / / www . mmm . ucar. $\mathrm{edu} / \mathrm{mm} 5 /$.

[12] "Global forecast system." www.emc.ncep.noaa.gov/index.php? branch=GFS.

[13] "Weather research and forecasting." http://www.wrf-model.org/ index.php.

[14] A. Papoulis and S. Pillai, Probability, Random Variables and Stochastic Processes. McGraw - Hill, fourth ed., January 2002.

[15] C. S. Gibbons J.D., Nonparametric Statistical Inference. Boca Raton, FL: CRC Press, fifth ed., 2011.

[16] J. Zhang, "Power system transmission line thermal overload risk assessment," Master's thesis, Iowa State University, 1999.

[17] T. Ackermann, Wind Power in Power Systems. Wiley, 1st ed., September 2005.

[18] M. Lange and U. Focken, "New developments in wind energy forecasting," in Power and Energy Society General Meeting - Conversion and Delivery of Electrical Energy in the 21st Century, 2008 IEEE, july 2008.

[19] C. Potter and M. Negnevitsky, "Very short-term wind forecasting for tasmanian power generation,” Power Systems, IEEE Transactions on, vol. 21, pp. 965 - 972, may 2006. 
[20] A. More and M. Deo, "Forecasting wind with neural networks," Elsevier Journal of Marine Structures, vol. 16, pp. 35 -49, 2003.

[21] G. Kariniotakis, G. Stavrakakis, and E. Nogaret, "Wind power forecasting using advanced neural networks models," Energy Conversion, IEEE Transactions on, vol. 11, pp. $762-767$, dec 1996.

[22] A. Sfetsos, "A comparison of various forecasting techniques applied to mean hourly wind-speed time series," Elsevier Journal of Renewable Energy, vol. 21, pp. $21-35,2000$.

[23] W. Y.-H. Milligan M., Schwartz M., "Statistical wind power forecasting models: Results foru.s. wind farms." http://www.nrel.gov/docs/fy03osti/ 33956 .pdf, May 2003.

[24] B. Ernst, B. Oakleaf, M. Ahlstrom, M. Lange, C. Moehrlen, B. Lange, U. Focken, and K. Rohrig, "Predicting the wind," Power and Energy Magazine, IEEE, vol. 5, pp. $78-89$, nov.-dec. 2007.

[25] "World wind power predictions." http://www.windpowerpredictions. $\mathrm{com} /$.

[26] "Wind power prediction tool." http://www.enfor.eu/wind_power_ prediction_tool_wppt.php.

[27] M. Haque, "Efficient load flow method for distribution systems with radial or mesh configuration," Generation, Transmission and Distribution, IEE Proceedings-, vol. 143, pp. 33 -38, January 1996.

[28] M. Kamh and R. Iravani, "Unbalanced model and power-flow analysis of microgrids and active distribution systems," Power Delivery, IEEE Transactions on, vol. 25, pp. $2851-2858$, October 2010.

[29] Y. Atwa, E. El-Saadany, M. Salama, and R. Seethapathy, "Optimal renewable resources mix for distribution system energy loss minimization," Power Systems, IEEE Transactions on, vol. 25, pp. 360 -370, February 2010. 
[30] E. Coster, J. Myrzik, B. Kruimer, and W. Kling, "Integration issues of distributed generation in distribution grids," Proceedings of the IEEE, vol. 99, pp. $28-39$, January 2011.

[31] F. Abu-Mouti and M. El-Hawary, "Heuristic curve-fitted technique for distributed generation optimisation in radial distribution feeder systems," Generation, Transmission Distribution, IET, vol. 5, pp. 172 -180, February 2011.

[32] J. Northcote-Green and R. Wilson, Control and Automation of Electric Power Distribution Systems. CRC Press, September 2006.

[33] W. Yang and J. Jiang, "Wind turbine condition monitoring and reliability analysis by scada information," in Mechanic Automation and Control Engineering (MACE), 2011 Second International Conference on, pp. 1872 -1875, July 2011.

[34] W. Yang, P. Tavner, C. Crabtree, and M. Wilkinson, "Cost-effective condition monitoring for wind turbines," Industrial Electronics, IEEE Transactions on, vol. 57, pp. $263-271$, Jan 2010.

[35] G. Smith, "Developement of a generic wind farm scada system," tech. rep., DTI Sustainable Energy Programmes, 2001.

[36] T. Gönen, Electric Power Distribution System Engineering. CRC Press, second ed., December 2007.

[37] B. J. L., Protective Relaying: Principles and Applications. Boca Raton, FL: CRC Press, third ed., 2007.

[38] W. Kersting, Distribution System Modeling and Analysis. CRC Press, second ed., November 2006.

[39] S. Civanlar, J. Grainger, H. Yin, and S. Lee, "Distribution feeder reconfiguration for loss reduction," Power Delivery, IEEE Transactions on, vol. 3, pp. 1217 -1223, July 1988. 
[40] P. Barker and R. De Mello, "Determining the impact of distributed generation on power systems, part i - radial distribution systems," in Power Engineering Society Summer Meeting, 2000. IEEE, vol. 3, pp. 1645 -1656 vol. 3, 2000.

[41] D. Shirmohammadi and H. Hong, "Reconfiguration of electric distribution networks for resistive line losses reduction," Power Delivery, IEEE Transactions on, vol. 4, pp. 1492 -1498, April 1989.

[42] C. Liu, S. Lee, and K. Vu, "Loss minimization of distribution feeders: optimality and algorithms," Power Delivery, IEEE Transactions on, vol. 4, pp. 1281 -1289, apr 1989.

[43] M. Baran and F. Wu, "Network reconfiguration in distribution systems for loss reduction and load balancing," Power Delivery, IEEE Transactions on, vol. 4, pp. 1401 -1407, April 1989.

[44] J. Zhu, D. Lubkeman, and A. Girgis, "Automated fault location and diagnosis on electric power distribution feeders," Power Delivery, IEEE Transactions on, vol. 12, pp. 801 -809, April 1997.

[45] Y. Liao, "Generalized fault-location methods for overhead electric distribution systems," Power Delivery, IEEE Transactions on, vol. 26, pp. 53 -64, January 2011.

[46] M. Mousavi and K. Butler-Purry, "Detecting incipient faults via numerical modeling and statistical change detection," Power Delivery, IEEE Transactions on, vol. 25, pp. $1275-1283$, July 2010.

[47] W.-H. Chen, "Fault section estimation using fuzzy matrix-based reasoning methods," Power Delivery, IEEE Transactions on, vol. 26, pp. 205 -213, January 2011.

[48] Y. Zhang, M. Ilic and, and O. Tonguz, "Mitigating blackouts via smart relays: A machine learning approach," Proceedings of the IEEE, vol. 99, pp. $94-118$, January 2011.

[49] “Confrrm: Solar energy data resource." http://rredc.nrel.gov/solar/ new_data/confrrm/. 
[50] G. J. Safari B., "A statistical investigation of wind characteristics and wind energy potential based on the weibull and rayleigh models in rwanda," Elsevier Renewable Energy: An Internation Journal, pp. 2874-2880, 2010.

[51] F. K. A. Olaofe, Z. O., "Statistical analysis of wind resources at darling for energy production," INTERNATIONAL JOURNAL of RENEWABLE ENERGY RESEARCH, vol. Second, 2012.

[52] "Wind resource assessment handbook," tech. rep., National Renewable Energy Laboratory, Golden, CO, April 1997. 


\section{Appendix A}

\section{Simulation Setup and Detailed Results}

\section{A.1 Wind Data Analysis}

This section will cover the results of the probability calculation. Results covered in this section are examples of normalized frequency plots and relevant calculated Weibull density functions. The results of calculation of $k$ and $c$ parameters for parametric calculation are tabulated. Probabilities calculated using both parametric and nonparametric approach are also tabulated. MATLAB statistical toolbox was used for all calculations in this section.

\section{A.2 Frequency Plots for Various Time-frames}

Data of each individual year was taken and normalized frequency plots are obtained. Similarly, all four years of data was also divided into four seasons (See Figure 3.3). The number of total data points over a year is significantly large $(105,120$ points for years 2007, 2009, 2010 and 105,408 for the year 2008) for both seasonal and annual plots. Figure A.2 shows the frequency plot for data points over each anum, while figure A.1 shows similar plots for each season. 

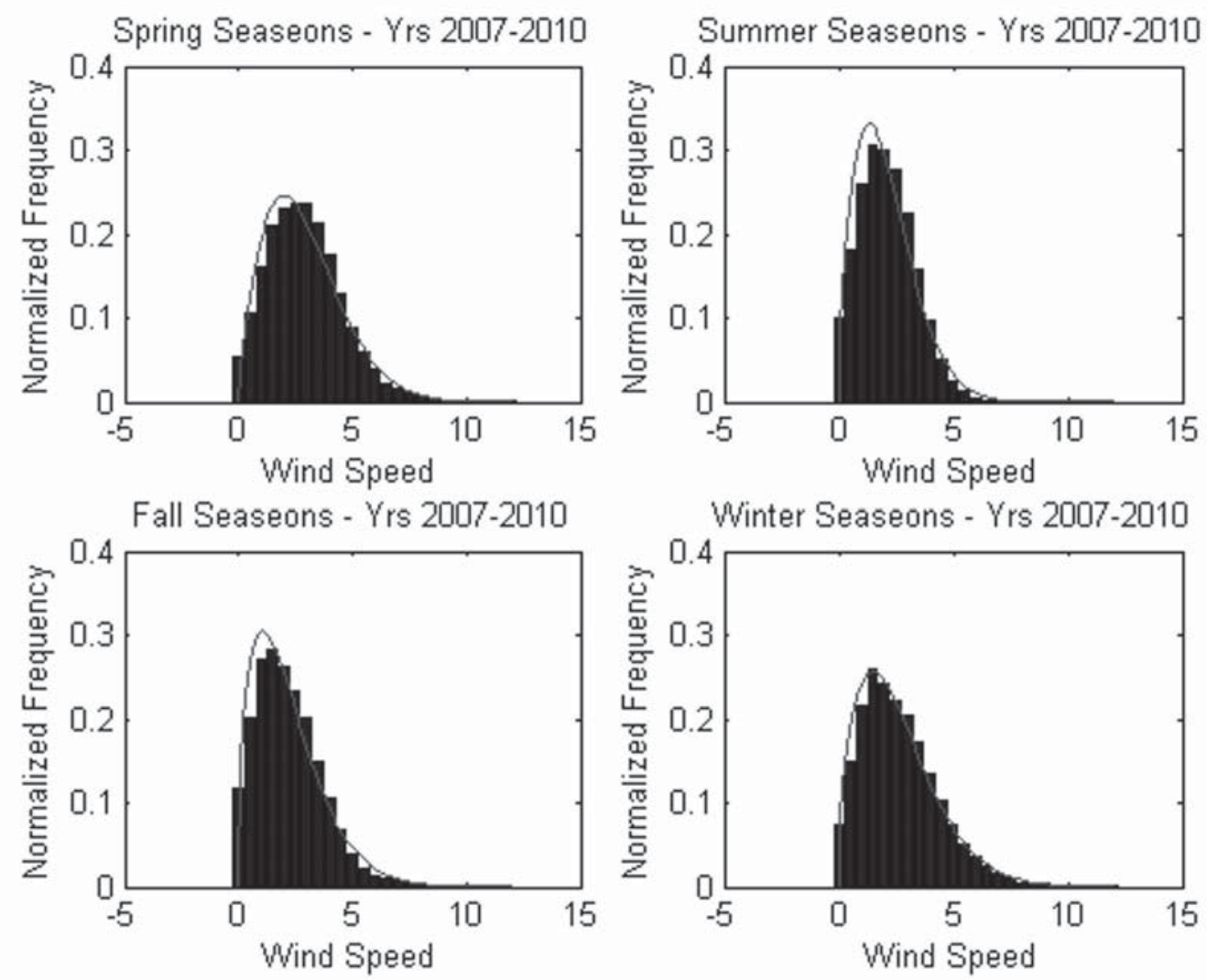

Figure A.1 - Individual Normalized Frequency Plots for Each Season

\section{A.3 Wind Probability Calculation}

After segregating the data into desired time-frames, probability calculations were made. For parametric approach, $k$ and $c$ parameters are calculated for each set of data. Division of data is shown in Figure 3.3. Using Maximum likelihood estimation for each of the data set, best fitting parameters for Weibull PDF were obtained. The PDF expressions (See Equation (3.8)) thus calculated can be superimposed on top of the normalized frequency plots (as in Figure A.2) for a visual inspection of how well the data fits the density function. Table A.1 shows the values obtained from MLE for data points in each hour and every season. By integrating the PDF expressions (See Equation (3.6) and (3.7)) for a desired range, probability of wind speed in that range can be calculated.

Table A. 2 shows the probabilities of each wind speed at a randomly chosen hour 

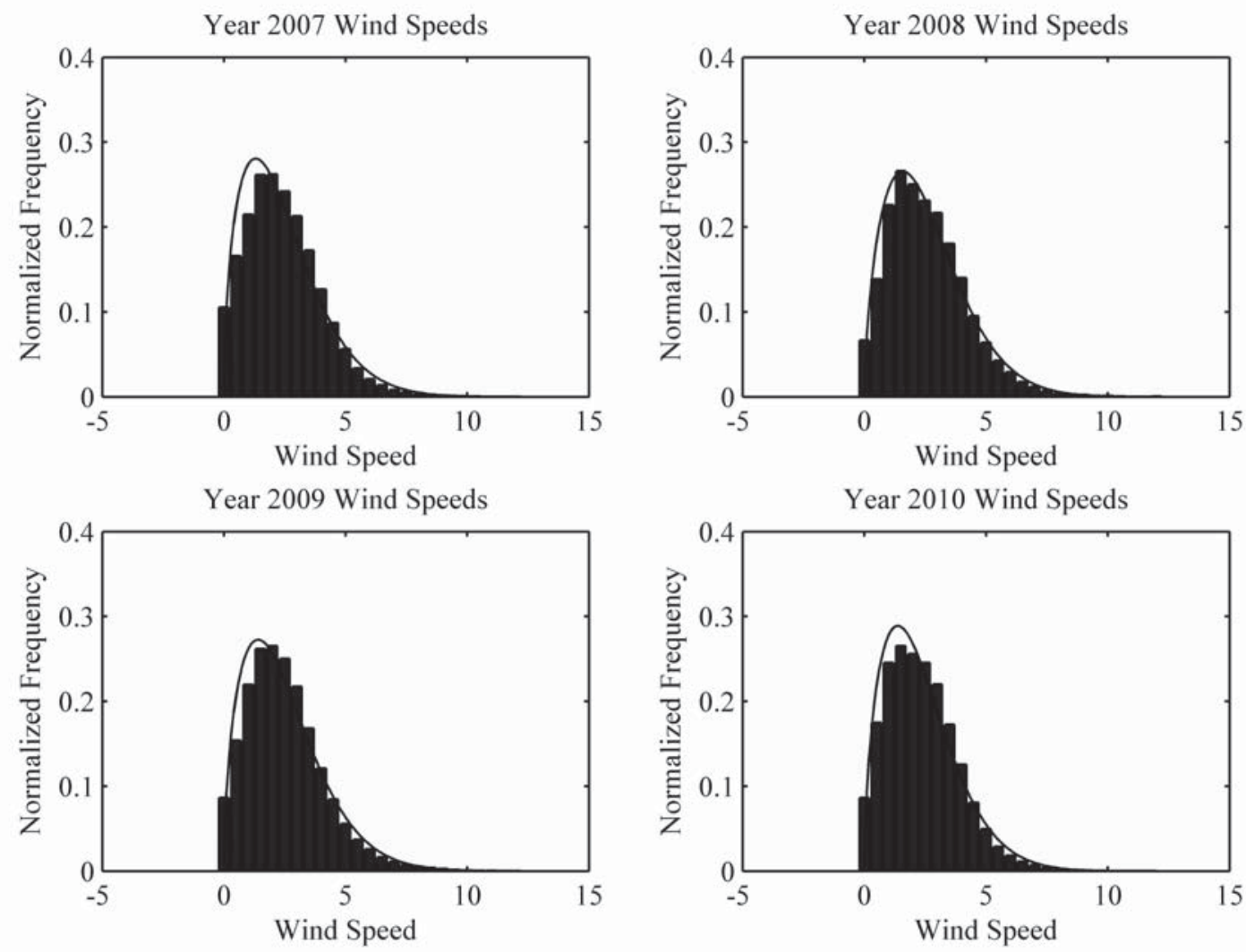

Figure A.2 - Individual Normalized Frequency Plots for Each Year

(12:00 midnight to 1:00AM) for all four seasons. Columns 2,4,6, and 8 show the results for most likely wind speeds. This was obtained by integrating the PDF expression around the peak of Weibull PDF obtained. Other probabilities can be obtained in a similar way by integrating over the desired portion of the PDF.

Columns 3,5,7, and 9 show results obtained from nonparametric calculation of probabilities (See Equation (3.12)). Here, the bin of speeds that had the highest number of occurrence were taken as the most probable wind speeds and their probabilites were calculated. Similar to parametric calculation, probabilities of a range of wind speed can be obtained by using the total number of points in the desired range to calculate the total probability. 
Table A.1

Calculated $k$ and $c$ Parameters

\begin{tabular}{|c|c|c|c|c|c|c|c|c|}
\hline \multirow[b]{2}{*}{$\begin{array}{l}\text { Hour of } \\
\text { Day }\end{array}$} & \multicolumn{2}{|c|}{ Spring } & \multicolumn{2}{|c|}{ Summer } & \multicolumn{2}{|c|}{ Fall } & \multicolumn{2}{|c|}{ Winter } \\
\hline & $k$ & $c$ & $k$ & $c$ & $k$ & $c$ & $k$ & $c$ \\
\hline Midnight & 2.4898 & 1.5124 & 1.5780 & 1.3479 & 1.8026 & 1.2299 & 2.4218 & 1.4342 \\
\hline 1:00AM & 2.3975 & 1.4269 & 1.5662 & 1.3285 & 1.7944 & 1.2071 & 2.3452 & 1.3990 \\
\hline 2:00AM & 2.3393 & 1.4855 & 1.4947 & 1.3642 & 1.7841 & 1.1968 & 2.4482 & 1.4312 \\
\hline 3:00AM & 2.3496 & 1.4991 & 1.4882 & 1.3404 & 1.8096 & 1.2463 & 2.5344 & 1.5290 \\
\hline 4:00AM & 2.3310 & 1.4852 & 1.4565 & 1.3402 & 1.8022 & 1.2557 & 2.5331 & 1.5038 \\
\hline 5:00AM & 2.3032 & 1.4531 & 1.4464 & 1.2912 & 1.7882 & 1.1477 & 2.5066 & 1.4791 \\
\hline 6:00AM & 2.5380 & 1.5401 & 1.7286 & 1.4389 & 1.8571 & 1.1995 & 2.4091 & 1.4508 \\
\hline 7:00AM & 3.0879 & 1.7764 & 2.2411 & 1.7724 & 2.1408 & 1.3737 & 2.4763 & 1.4675 \\
\hline 8:00AM & 3.6376 & 2.0608 & 2.6094 & 2.1834 & 2.6651 & 1.5892 & 2.9317 & 1.6853 \\
\hline 9:00AM & 3.9053 & 2.2226 & 2.7955 & 2.3354 & 3.0832 & 1.8529 & 3.4665 & 1.8852 \\
\hline 10:00AM & 4.0263 & 2.3099 & 2.9010 & 2.4953 & 3.3129 & 2.0338 & 3.8192 & 2.0196 \\
\hline 11:00AM & 4.1197 & 2.3243 & 3.0172 & 2.5822 & 3.4555 & 2.1830 & 3.9988 & 2.0994 \\
\hline Noon & 4.2584 & 2.3697 & 3.1669 & 2.7016 & 3.5227 & 2.3331 & 4.0923 & 2.1528 \\
\hline 1:00PM & 4.3979 & 2.4883 & 3.2829 & 2.7931 & 3.5290 & 2.3164 & 4.0524 & 2.0727 \\
\hline 2:00PM & 4.4783 & 2.5650 & 3.3880 & 2.6966 & 3.4837 & 2.3699 & 3.9284 & 2.0337 \\
\hline 3:00PM & 4.4126 & 2.6333 & 3.3787 & 2.7222 & 3.3352 & 2.3386 & 3.6790 & 2.0243 \\
\hline 4:00PM & 4.1892 & 2.7275 & 3.2942 & 2.7997 & 2.8759 & 2.0306 & 3.2915 & 1.8932 \\
\hline 5:00PM & 3.6966 & 2.5460 & 2.9816 & 2.6215 & 2.3462 & 1.7077 & 2.8134 & 1.6254 \\
\hline 6:00PM & 3.1267 & 2.1581 & 2.5134 & 2.2407 & 2.0324 & 1.5012 & 2.5083 & 1.4571 \\
\hline 7:00PM & 2.7954 & 1.9122 & 2.0871 & 1.8820 & 1.8796 & 1.3804 & 2.4382 & 1.4239 \\
\hline 8:00PM & 2.6732 & 1.7611 & 1.9280 & 1.7052 & 1.8500 & 1.3626 & 2.3638 & 1.3656 \\
\hline 9:00PM & 2.6569 & 1.6889 & 1.7813 & 1.5765 & 1.7714 & 1.2884 & 2.3849 & 1.3500 \\
\hline 10:00PM & 2.5950 & 1.5983 & 1.7180 & 1.4989 & 1.7741 & 1.1867 & 2.3354 & 1.3694 \\
\hline 11:00PM & 2.4922 & 1.4977 & 1.6263 & 1.4237 & 1.8205 & 1.2256 & 2.4047 & 1.4082 \\
\hline
\end{tabular}

\section{A.4 Wind Power Calculation}

Table A.3 shows the parameters used to obtain the simulated power curve shown in 4.3. The values shown were used to calculate the power output from an individual $1.5 \mathrm{MW}$ turbine. The curve in Figure 4.3 was also obtained using the power output calculated above.

Table A.4 summarizes the wind speeds observed in our data and adjusted wind speeds. 
Table A.2

Wind Speeds and Respective Probabilities for the Same Time of Day in a Season

\begin{tabular}{lllllllll}
\hline \multicolumn{2}{c}{ Spring } & \multicolumn{2}{c}{ Summer } & \multicolumn{2}{c}{ Fall } & \multicolumn{2}{c}{ Winter } \\
\hline $\begin{array}{l}\text { Wind } \\
\text { Speed } \\
(m / s)\end{array}$ & Weibull & $\begin{array}{l}\text { Non- } \\
\text { parametric }\end{array}$ & Weibull & $\begin{array}{l}\text { Non- } \\
\text { parametric }\end{array}$ & Weibull & $\begin{array}{l}\text { Non- } \\
\text { parametric }\end{array}$ & Weibull & $\begin{array}{l}\text { Non- } \\
\text { parametric }\end{array}$ \\
\hline $\mathbf{0 . 0 0}$ & 0.0832 & 0.0482 & 0.1887 & 0.1088 & 0.1839 & 0.0977 & 0.0974 & 0.0530 \\
$\mathbf{0 . 5 0}$ & 0.1378 & 0.0895 & 0.2265 & 0.1646 & 0.1976 & 0.1554 & 0.1461 & 0.1039 \\
$\mathbf{1 . 0 0}$ & 0.1492 & 0.1393 & 0.1898 & 0.2049 & 0.1660 & 0.1923 & 0.1502 & 0.1574 \\
$\mathbf{1 . 5 0}$ & 0.1407 & 0.1548 & 0.1410 & 0.1849 & 0.1297 & 0.1500 & 0.1372 & 0.1450 \\
$\mathbf{2 . 0 0}$ & 0.1223 & 0.1325 & 0.0971 & 0.1291 & 0.0971 & 0.1406 & 0.1168 & 0.1136 \\
$\mathbf{2 . 5 0}$ & 0.1002 & 0.1057 & 0.0633 & 0.0928 & 0.0705 & 0.1033 & 0.0945 & 0.1146 \\
$\mathbf{3 . 0 0}$ & 0.0783 & 0.0991 & 0.0394 & 0.0588 & 0.0500 & 0.0528 & 0.0736 & 0.0956 \\
$\mathbf{3 . 5 0}$ & 0.0588 & 0.0787 & 0.0236 & 0.0317 & 0.0347 & 0.0321 & 0.0554 & 0.0640 \\
$\mathbf{4 . 0 0}$ & 0.0426 & 0.0623 & 0.0137 & 0.0152 & 0.0237 & 0.0230 & 0.0405 & 0.0499 \\
$\mathbf{4 . 5 0}$ & 0.0299 & 0.0388 & 0.0077 & 0.0053 & 0.0160 & 0.0173 & 0.0289 & 0.0348 \\
$\mathbf{5 . 0 0}$ & 0.0204 & 0.0193 & 0.0042 & 0.0015 & 0.0106 & 0.0140 & 0.0201 & 0.0258 \\
$\mathbf{5 . 5 0}$ & 0.0136 & 0.0113 & 0.0023 & 0.0008 & 0.0070 & 0.0071 & 0.0137 & 0.0151 \\
$\mathbf{6 . 0 0}$ & 0.0088 & 0.0066 & 0.0012 & 0.0005 & 0.0046 & 0.0041 & 0.0092 & 0.0131 \\
$\mathbf{6 . 5 0}$ & 0.0056 & 0.0049 & 0.0006 & 0.0008 & 0.0029 & 0.0013 & 0.0060 & 0.0063 \\
$\mathbf{7 . 0 0}$ & 0.0035 & 0.0016 & 0.0003 & 0.0003 & 0.0019 & 0.0028 & 0.0039 & 0.0034 \\
$\mathbf{7 . 5 0}$ & 0.0021 & 0.0021 & 0.0001 & 0.0000 & 0.0012 & 0.0026 & 0.0025 & 0.0017 \\
$\mathbf{8 . 0 0}$ & 0.0013 & 0.0005 & 0.0001 & 0.0000 & 0.0007 & 0.0018 & 0.0015 & 0.0007 \\
$\mathbf{8 . 5 0}$ & 0.0007 & 0.0014 & 0.0000 & 0.0000 & 0.0005 & 0.0013 & 0.0010 & 0.0005 \\
$\mathbf{9 . 0 0}$ & 0.0004 & 0.0014 & 0.0000 & 0.0000 & 0.0003 & 0.0005 & 0.0006 & 0.0007 \\
$\mathbf{9 . 5 0}$ & 0.0002 & 0.0012 & 0.0000 & 0.0000 & 0.0002 & 0.0000 & 0.0003 & 0.0007 \\
$\mathbf{1 0 . 0 0}$ & 0.0001 & 0.0007 & 0.0000 & 0.0000 & 0.0001 & 0.0000 & 0.0002 & 0.0002 \\
$\mathbf{1 0 . 5 0}$ & 0.0001 & 0.0002 & 0.0000 & 0.0000 & 0.0001 & 0.0000 & 0.0001 & 0.0000 \\
$\mathbf{1 1 . 0 0}$ & 0.0000 & 0.0000 & 0.0000 & 0.0000 & 0.0000 & 0.0000 & 0.0001 & 0.0000 \\
$\mathbf{1 1 . 5 0}$ & 0.0000 & 0.0000 & 0.0000 & 0.0000 & 0.0000 & 0.0000 & 0.0000 & 0.0000 \\
$\mathbf{1 2 . 0 0}$ & 0.0000 & 0.0000 & 0.0000 & 0.0000 & 0.0000 & 0.0000 & 0.0000 & 0.0000 \\
\hline & & & & & & & & \\
\hline
\end{tabular}

\section{A.5 Test System Setup}

This section covers details on the test system (See Figure 5.2) simulated in MATLAB Simulink, using Simpowersystems toolbox. The system was initially built with just one wind turbine of $1.5 \mathrm{MW}$ capacity, as shown in A.3. This is loosely based on the MATLAB simulation demo power wind dfig. Important building blocks of the system are the wind turbine protection block and a wind turbine monitoring system. 
Table A.3

Parameters Used to Calculate Individual Turbine Output

\begin{tabular}{ll}
\hline Parameter & Value \\
\hline Cut-in Speed, $V_{C I}$ & $4 \mathrm{~m} / \mathrm{s}$ \\
Cut-off Speed, $V_{C O}$ & $26 \mathrm{~m} / \mathrm{s}$ \\
Rated Speed, $V_{R}$ & $14 \mathrm{~m} / \mathrm{s}$ \\
Measuring Mast Height, $Z_{i}$ & $10 \mathrm{~m}$ \\
Hub Height, $Z$ & $65 \mathrm{~m}$ \\
Length of Blades, $r$ & $30 \mathrm{~m}$ \\
Air Density, $\rho$ & $1.225 \mathrm{~kg} / \mathrm{m}^{3}$ \\
Coefficient of Performance, $C_{P}$ & 0.35 \\
Equivalent Efficiency, $\eta_{e q}$ & 0.7 \\
Hellman's Coefficient & 0.5 \\
\hline
\end{tabular}

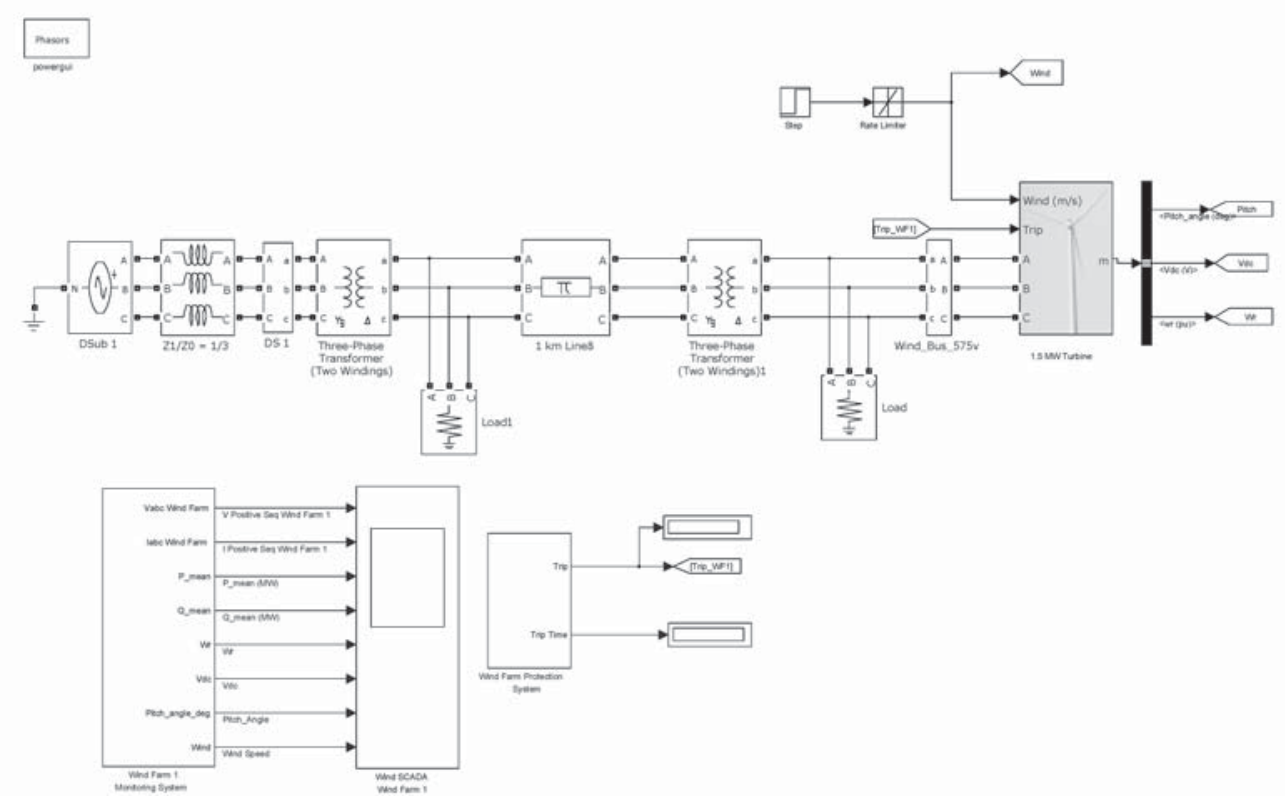

Figure A.3 - Base Case MATLAB Configuration

Complexity was added in the form of addition of other loads and lines along with measurements at the grid side. This was furthered by adding two more feeders DS-2 
Table A.4

Adjusted Wind Speeds and Respective Power Outputs for a Hub-height of 60m

\begin{tabular}{llllll}
\hline $\begin{array}{l}\text { Mast } \\
\text { Wind } \\
\begin{array}{l}\text { Speed } \\
(\mathrm{m} / \mathrm{s})\end{array}\end{array}$ & $\begin{array}{l}\text { Adjusted } \\
\text { Wind } \\
\text { Speed } \\
(\mathrm{m} / \mathrm{s})\end{array}$ & $\begin{array}{l}\text { Calculated } \\
\text { Power } \\
\text { Output } \\
(\mathrm{MW})\end{array}$ & $\begin{array}{l}\text { Mast } \\
\text { Wind } \\
\text { Speed } \\
(\mathrm{m} / \mathrm{s})\end{array}$ & $\begin{array}{l}\text { Adjusted } \\
\text { Wind } \\
\text { Speed } \\
(\mathrm{m} / \mathrm{s})\end{array}$ & $\begin{array}{l}\text { Calculated } \\
\text { Power } \\
\text { Output } \\
(\mathrm{MW})\end{array}$ \\
\hline 0 & 0 & 0 & 6.00 & 15.2970 & 1.5 \\
0.50 & 1.3693 & 0 & 6.50 & 16.5718 & 1.5 \\
1.00 & 2.7386 & 0 & 7.00 & 17.8465 & 1.5 \\
1.50 & 4.1079 & 0 & 7.50 & 19.1213 & 1.5 \\
2.00 & 5.0990 & 0.0941 & 8.00 & 20.3961 & 1.5 \\
2.50 & 6.3738 & 0.1838 & 8.50 & 21.6708 & 1.5 \\
3.00 & 7.6485 & 0.3177 & 9.00 & 22.9456 & 1.5 \\
3.50 & 8.9233 & 0.5045 & 9.50 & 24.2203 & 1.5 \\
4.00 & 10.198 & 0.5510 & 10.00 & 25.4951 & 1.5 \\
4.50 & 11.473 & 0.7531 & 10.50 & 26.7698 & 0 \\
5.00 & 12.747 & 1.0722 & 11.00 & 28.0446 & 0 \\
5.50 & 14.0223 & 1.5 & 11.50 & 29.3193 & 0 \\
\hline
\end{tabular}

and DS-3, and another smaller wind farm at DS-3. Parameters for voltage levels chosen were loosely based on the IEEE 34 node standard system. 


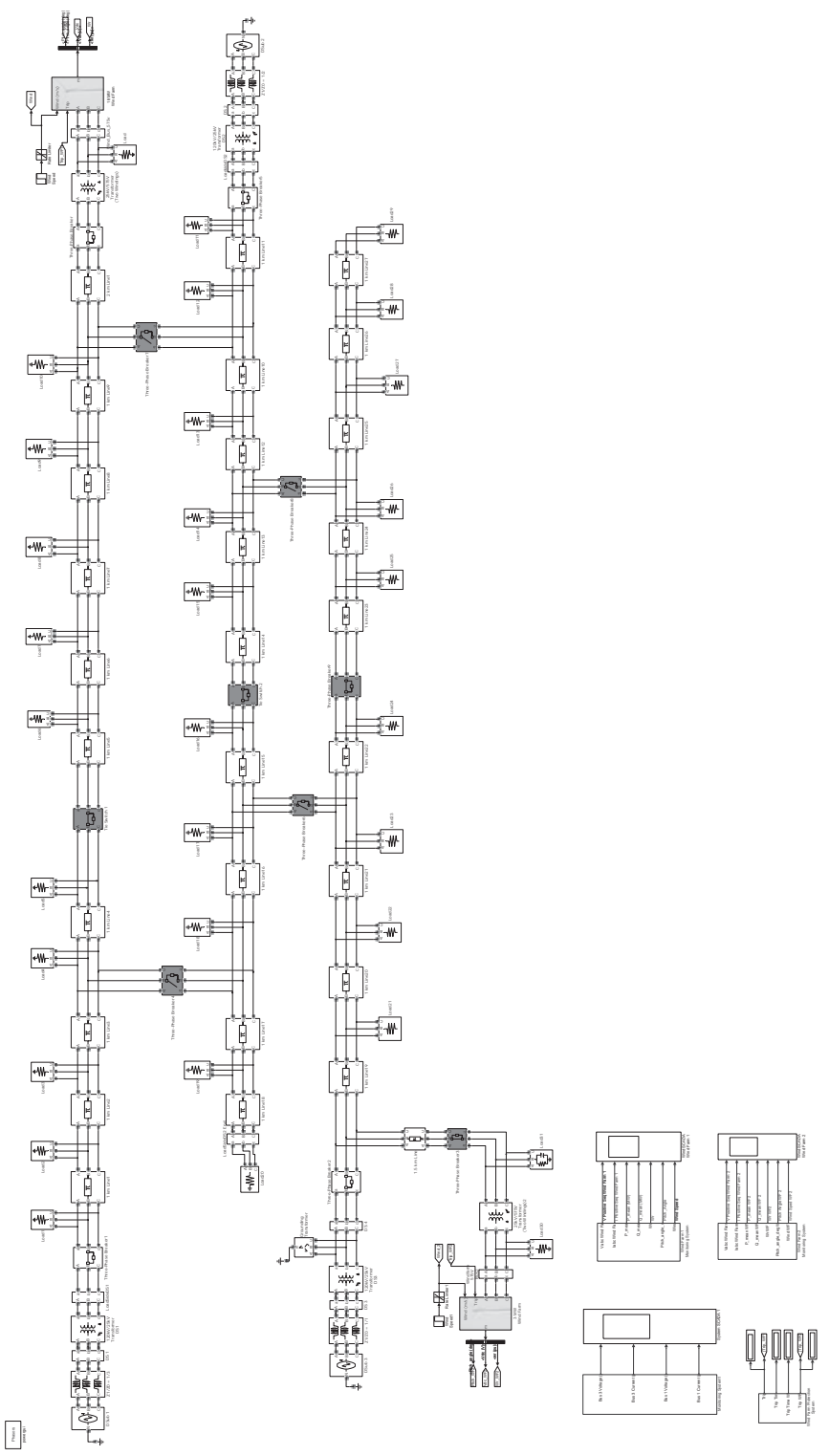

 
Test system shown in Figure 5.2 was simulated and Figure A.4 shows the arrangement of components in MATLAB SimPowerSystems. This system has two wind farms and three main feeders. Various parameters and components chosen for this system are indicated subsequent sections. The chosen solver, Load data, line data, generation data, monitoring and protection blocks for both the wind farms- 1 and 2 along with monitoring system on the grid part are discussed in detail in order for the reader to be able to replicate the results obtained in this thesis. To summarize, the test case simulated in MATLAB has the following components:

- Feeders, loads, lines, switches, and wind farms

- Distribution substation monitoring

- Wind farm monitoring

- Wind farm protection system

- Tie switches

\section{A.6 Solver}

The parameters fed to the test system (See Figure A.4) are shown in Tables A.7, A.8 and A.9. A dynamic simulation is run for varying time-periods, which solves for voltages and currents at various measuring points across the system. The solver used here is ODE23tb, which is used to solve for stiff systems (A stiff systems can be loosely defined as a system that has different time scales). The solver uses a variable step solver that changes the step-size automatically. Using ODE23tb as a solver has shown to reduce simulation time significantly. (Approx. 69.607634s for one simulation in ODE23tb as opposed to 856.246474s in ODE45 non-stiff solver for the exact same simulation).

\section{A.7 Duration of Simulation}

Since this work focused on operational aspects, total time taken for simulation is important. The factors affecting the duration of simulation are listed in A.5. Some of the aspects affecting the time taken are: 
1. The size of the system

2. The solver used

3. The computational power of the machine

Table A.5

Time Duration for Simulation

\begin{tabular}{ll}
\hline Simulation Stage & Time(Seconds) \\
\hline Historical data analysis & 59.303484 \\
Probability calculation & 0.730750 \\
Test system simulation (each combination) & 20.259979 \\
\hline
\end{tabular}

Table A.5 shows the time taken for each stage of the simulation. The longest time goes in testing each of the switching combinations. For the simple test case used here, there are 8 feasible combinations and the total time shown here is the addition of the computational time for each combination. All of the simulations were run on an Intel ${ }^{\circledR}$ Core2 CPU with 2.40GHz speed, a 3GB RAM and Microsoft ( Windows XP.

Table A.6

Wind Farm Data

\begin{tabular}{lll}
\hline Attribute & Wind Farm-1 & Wind Farm-2 \\
\hline Total Capacity & $18 \mathrm{MW}$ & $9 \mathrm{MW}$ \\
Number of Turbines & 12 & 6 \\
Type of Generator & DFIG & DFIG \\
\hline
\end{tabular}

Table A.7

Load Data for Test System

\begin{tabular}{lllll}
\hline Nodes & $\begin{array}{l}\text { Voltage } \\
\text { Level }\end{array}$ & $\begin{array}{l}\text { Individual } \\
\text { Load }\end{array}$ & Total Load & Type \\
\hline $1-10$ & $25 \mathrm{kV}$ & $1.5 \mathrm{MW}$ Each & $15 \mathrm{MW}$ & Resistive \\
$11-20$ & $25 \mathrm{kV}$ & $3 \mathrm{MW}$ Each & $30 \mathrm{MW}$ & Resistive \\
$21-29$ & $25 \mathrm{kV}$ & $2.5 \mathrm{MW}$ Each & $22.5 \mathrm{MW}$ & Resistive \\
\hline
\end{tabular}


Table A.8

Generation Data for Test System

\begin{tabular}{lllllll}
\hline $\begin{array}{l}\text { Feeder } \\
\text { Name }\end{array}$ & $\begin{array}{l}\text { Generated } \\
\text { Voltage Level }\end{array}$ & $\begin{array}{l}\text { Feeder Voltage } \\
\text { Level }\end{array}$ & $P_{\text {Gen }}$ & $Q_{\text {min }}$ & $Q_{\max }$ & Type \\
\hline DS1 & $120 \mathrm{kV}$ & $25 \mathrm{kV}$ Each & inf & - inf & inf & Swing bus \\
DS2 & $120 \mathrm{kV}$ & $25 \mathrm{kV}$ Each & $32 \mathrm{MW}$ & $-16 \mathrm{MW}$ & $16 \mathrm{MW}$ & PV bus \\
DS3 & $120 \mathrm{kV}$ & $25 \mathrm{kV}$ Each & $25 \mathrm{MW}$ & $-15 \mathrm{MW}$ & $15 \mathrm{MW}$ & PV bus \\
\hline
\end{tabular}

Table A.9

Line Data for Test System

\begin{tabular}{llllllll}
\hline Nodes & $\begin{array}{l}\text { Segment } \\
\text { Length }\end{array}$ & $R_{0}(\Omega)$ & $R_{1}(\Omega)$ & $L_{0}(\mathrm{mH} / \mathrm{km})$ & $L_{1}(\mathrm{mH} / \mathrm{km})$ & $C_{0}(\mathrm{nF} / \mathrm{km})$ & $C_{1}(\mathrm{nF} / \mathrm{km})$ \\
\hline $1-10$ & $1 \mathrm{~km}$ & 0.1153 & 0.413 & 1.05 & 3.32 & 11.33 & 5.01 \\
$11-20$ & $1 \mathrm{~km}$ & 0.1153 & 0.413 & 1.05 & 3.32 & 11.33 & 5.01 \\
$21-29$ & $1 \mathrm{~km}$ & 0.1153 & 0.413 & 1.05 & 3.32 & 11.33 & 5.01 \\
\hline
\end{tabular}

\section{A.8 Monitoring and Protection Blocks}
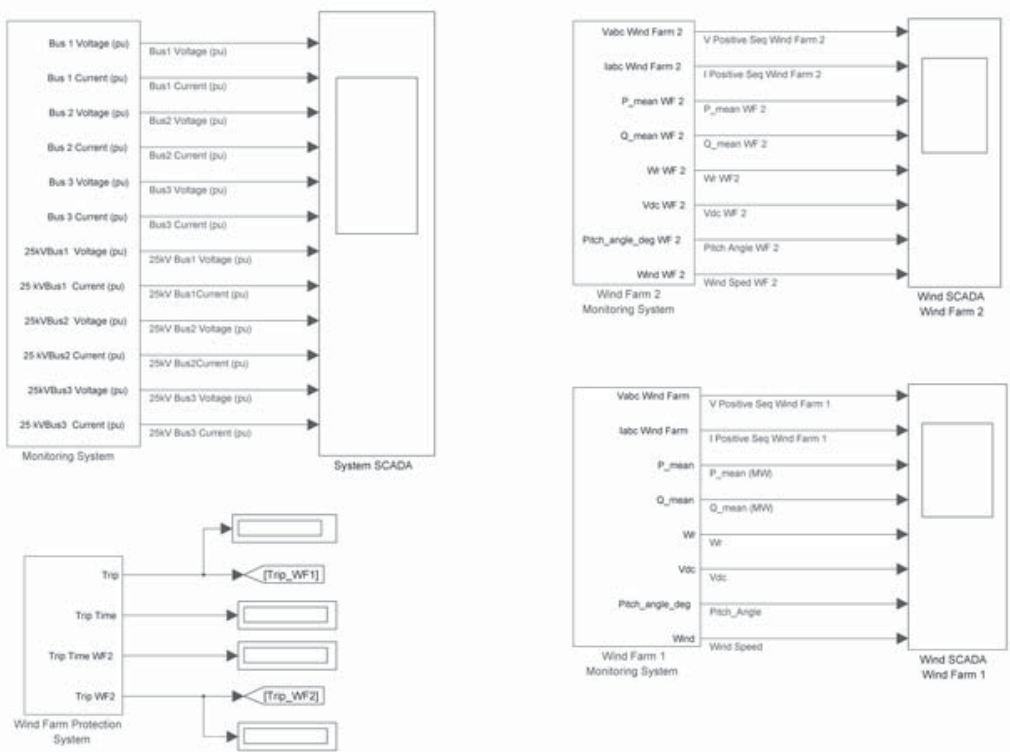

Figure A.5 - Monitoring and Control Blocks 
Figure A.5 shows all the monitoring blocks and control. Tags from measurement points around the system are referred to in these blocks. The blocks are then connected to scopes and number displays in order to monitor them.

\section{A.8.1 Distribution Substation Monitoring}

The test system has three distribution substations acting as swing buses. It is important to monitor the electrical quantities at these points. The distribution substation monitoring block of the system does that. Per unit values of line-to-ground voltage and current are measured for distribution substation monitoring. These measured values are then used to calculate active and reactive power at each point these measuring points are.

\section{A.8.2 Wind Farm Monitoring}

Each of the two wind farm has its own monitoring and control system. The required inputs to these systems are provided from the measuring points at each wind farm. $\mathrm{Pa}$ rameters measured in each such blocks are:

- Positive sequence voltage

- Positive sequence current

- Average real power, $P$

- Average reactive power, $Q$

- Wind speed, $v$

- Rotor speed, $w_{r}$

- Pitch angle of the blades

The monitoring system uses a measurement arrangement similar to distribution substation monitoring. Positive sequence components are calculated using these measured line-to-ground values and $a=1 \angle 120^{\circ}$. Average real and reactive power are calculated over 60 cycles. Other important characteristics monitored by this block include wind speed and corresponding rotor speeds along with pitch angle of blades. In order to maintain the nominal frequency, the wind model has partial frequency control using 
a two-way converter, which uses a capacitor as the DC source on the grid side. This voltage level is also important and hence monitored.

\section{A.8.3 Wind Farm Protection}

The protection block provides protection to wind turbines.

- Instantaneous AC overcurrent

- $\mathrm{AC}$ over current

- $\mathrm{AC}$ over current

- AC under voltage

- AC over voltage

- AC Voltage unbalance both positive and zero sequence

- DC over voltage

- Over speed

- Under speed

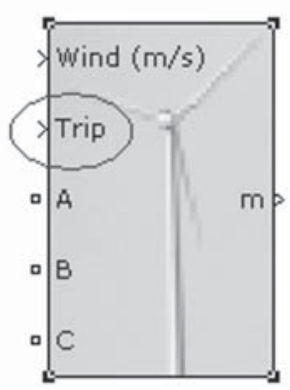

(a) Wind Turbine Model in MATLAB

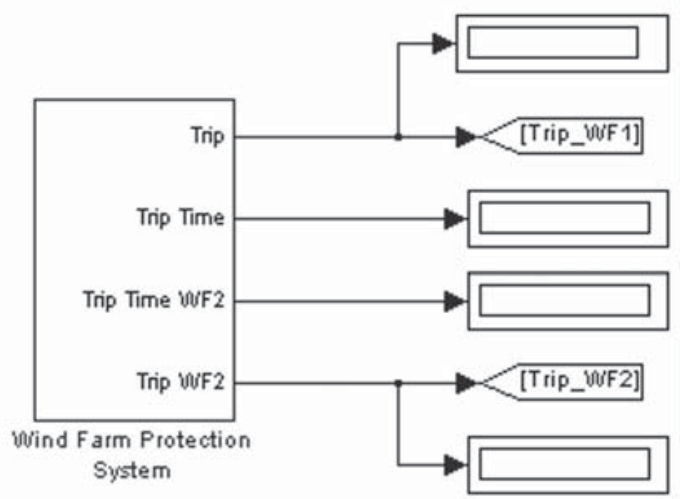

(b) Turbine Protection Block

Figure A.6 - Wind Turbine Protection

Figure A.6a in A.6 shows a wind turbine model block and as shown by circled part, it needs a trip signal as an input. This trip signal is fed by wind turbine protection block, 
which trips for the list of conditions mentioned above. Thresholds for each condition in the list are customizable. They are kept at values typically chosen in such blocks. The protection system gets inputs from the wind turbine monitoring system and the turbine block trips if any of the specified thresholds are violated.

\section{A.9 Simulation Results for Test System in MATLAB}

The test system built was tested for various scenarios discussed in Chapter 5. Relevant results are discussed below. The following is the discussion of some peculiarities were encountered in the MATLAB simulations, which will help the reader replicate the system easily.

- MATLAB model then that from calculations: The line connected to wind farm and the transformer next to it cannot be connected directly to a measuring block, as the simulation results in error. In order to avoid this error, a large resistor in form of a resistive ground was used. This is the only way I could find to make the turbine model work. I believe the dissipation occuring in this resistive block to be the reason for lower measured output of the wind farms (1.4714 instead of a full 1.5). I still find MATLAB simulink and its ways mysterious and keep finding new things about it.

- Figure A.7 shows that in absence of grid support, wind farm protection system is activated because of low voltage and the protection trips, removing the wind farm from operation. As mentioned in Section 5.2.3, it is normal utility practice to remove wind farms from operation in an event of fault. 

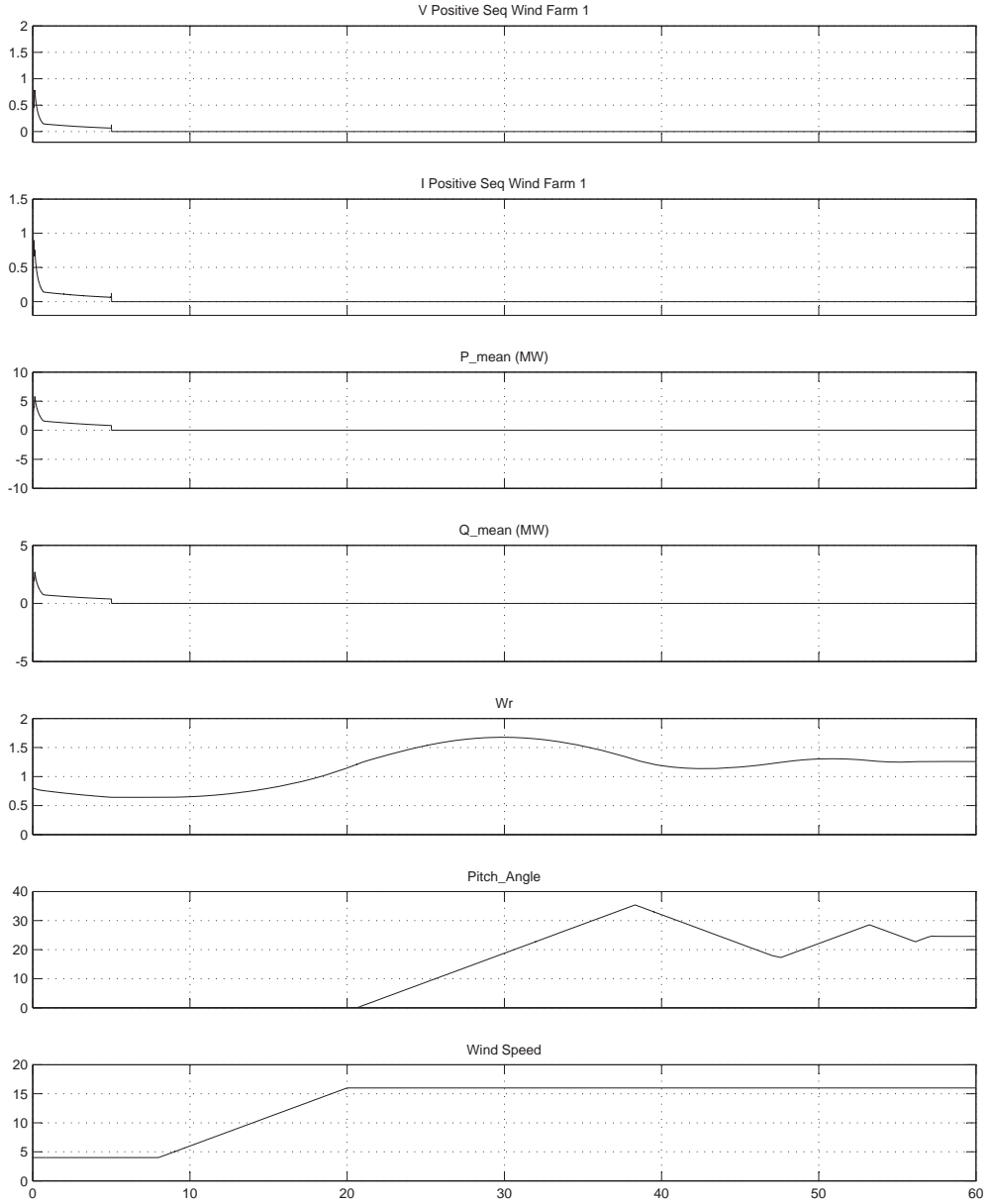

Time offset: 0

Figure A.7 - Wind Farm Behavior in Absence of Grid Support 